\title{
Schulbücher als Vermittler der Europäischen Integration?
}

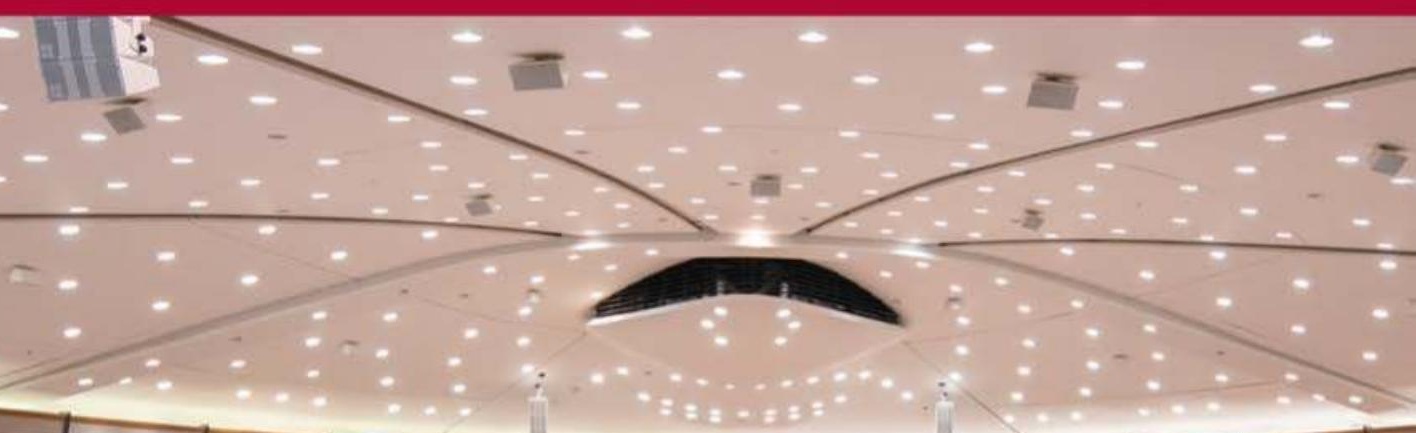

1)

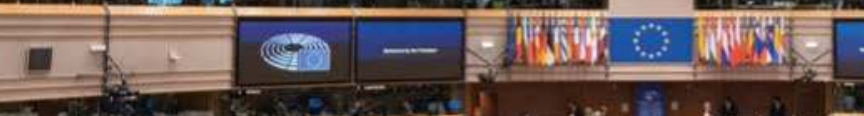

ब

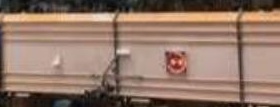

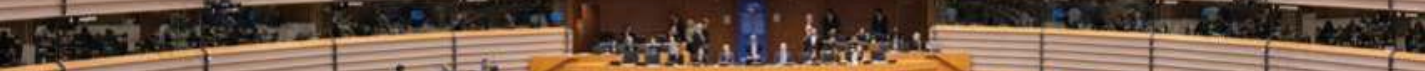

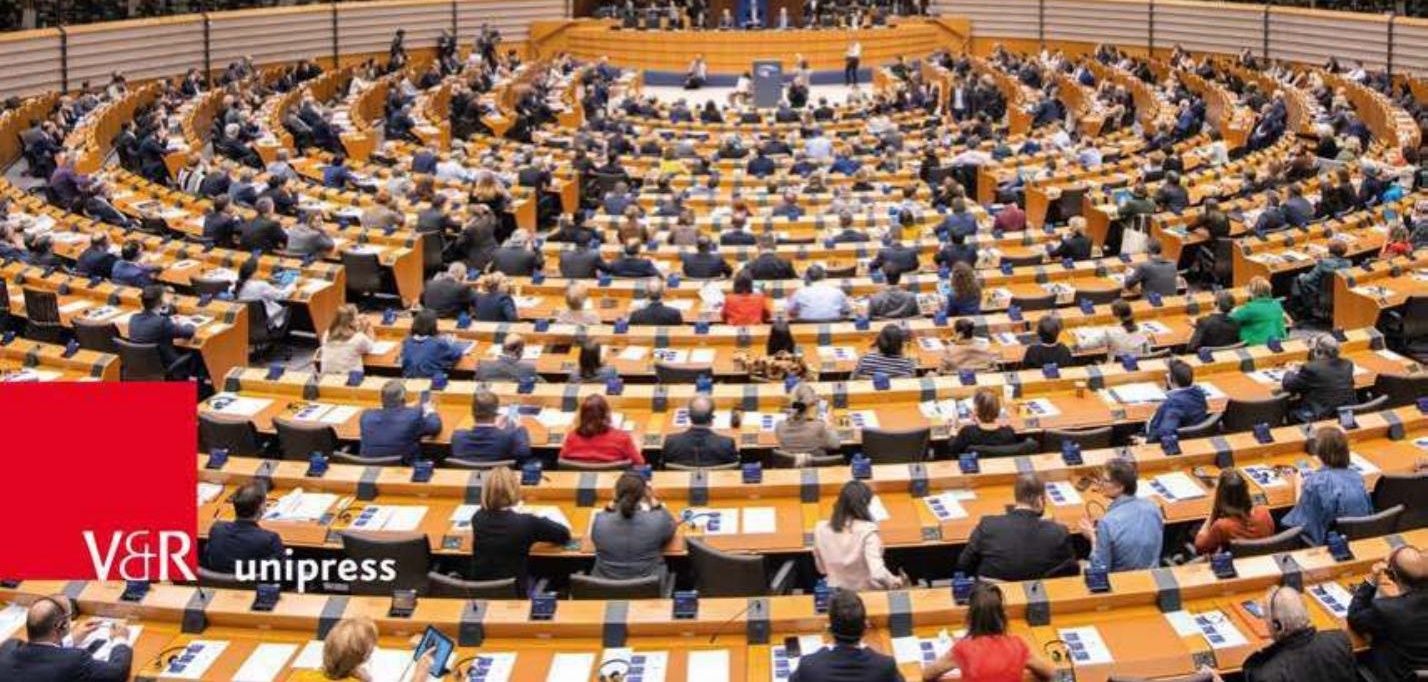




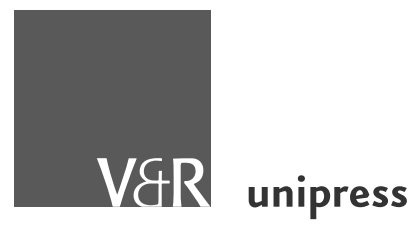

Open-Access-Publikation im Sinne der CC-Lizenz BY 4.0

(C) 2021, Vandenhoeck \& Ruprecht GmbH \& Co. KG, Göttingen ISBN Print: 9783847112655 - ISBN E-Lib: 9783737012652 


\section{Eckert. Expertise \\ Georg-Eckert-Institut für internationale Schulbuchforschung}

Band 10

Herausgegeben von

Eckhardt Fuchs

Redaktion

Wibke Westermeyer 


\section{Monika Oberle / Marret Bischewski / Christian Tatje}

\section{Schulbücher als Vermittler der Europäischen Integration?}

Eine produkt- und wirkungsorientierte Studie zum politischen Fachunterricht

Mit 24 Abbildungen

V\&R unipress 
Bibliografische Information der Deutschen Nationalbibliothek

Die Deutsche Nationalbibliothek verzeichnet diese Publikation in der Deutschen

Nationalbibliografie; detaillierte bibliografische Daten sind im Internet über https://dnb.de abrufbar.

Gefördert durch den Publikationsfonds für Open-Access-Monografien der Leibniz-Gemeinschaft.

(C) 2021, Vandenhoeck \& Ruprecht GmbH \& Co. KG, Theaterstraße 13, D-37073 Göttingen Dieses Werk ist als Open-Access-Publikation im Sinne der Creative-Commons-Lizenz BY International 4.0 (»Namensnennung«) unter dem DOI 10.14220/9783737012652 abzurufen. Um eine Kopie dieser Lizenz zu sehen, besuchen Sie https://creativecommons.org/licenses/ by/4.0/.

Jede Verwertung in anderen als den gesetzlich zugelassenen Fällen bedarf der vorherigen schriftlichen Einwilligung des Verlages.

Umschlagabbildung: EP-091103B; @ European Union 2019 - Source: EP; Fotografin: Emilie Gomez

Druck und Bindung: CPI books GmbH, Birkstraße 10, D-25917 Leck

Printed in the EU.

Vandenhoeck \& Ruprecht Verlage | www.vandenhoeck-ruprecht-verlage.com

ISSN 2198-1531

ISBN 978-3-7370-1265-2 


\section{Inhalt}

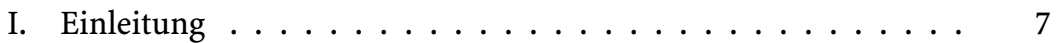

II. Die produktorientierte Teilstudie: Darstellung der Europäischen Union im Politikschulbuch . . . . . . . . . . 17

II.1 Fallauswahl und Analysemethode . . . . . . . . . . 18

II.2 Ergebnisse . . . . . . . . . . . . . . . . . . . 24

II.3 Zusammenfassung . . . . . . . . . . . 38

III. Die prozess- und wirkungsorientierte Teilstudie: Nutzung und Bewertung der EU-Kapitel durch Lehrkräfte sowie Schülerinnen und Schüler . . . . . . . . . . . . . . . . . . 43

III.1 Ziele und Fragestellungen . . . . . . . . . . . . . . . . . . . . 44

III.2 Design der Studie . . . . . . . . . . . . . . . . . . . . . . . 44

III.3 Ergebnisse zur Nutzung . . . . . . . . . . . . . . . . 46

III.4 Ergebnisse zur Bewertung . . . . . . . . . . . . 62

III.5 Detaillierte Bewertung des EU-Kapitels (Lehrkräfte) . . . . 65

III.6 Detaillierte Beurteilung des EU-Kapitels (Schülerinnen und Schüler $\ldots \ldots \ldots \ldots \ldots 6 \ldots$

III.7 Zusammenfassung . . . . . . . . . . . . . . . 68

IV. Fazit und Ausblick $\ldots \ldots \ldots \ldots$. . . . . . . . . 71 
V. Empfehlungen für die Darstellung der Europäischen Union in Politikschulbüchern . . . . . . . . . . . . . . . 77

VI. Literaturangaben zu den untersuchten Schulbüchern (DEUS 


\section{Einleitung}

Ohne Einbeziehung der europäischen Dimension lässt sich Politik in Deutschland heute weder angemessen begreifen noch gestalten. In der globalisierten Welt haben gesellschaftliche Probleme und nationalstaatliche Lösungsfähigkeit an Deckungsgleichheit verloren (vgl. Zürn 2005, 2018). Eine Antwort auf die Entgrenzung der Problemlagen ist die Übertragung nationaler Souveränität auf andere politische Ebenen und neue Akteure. Im Europäischen Mehrebenensystem war in den vergangenen Jahren und Jahrzehnten eine kontinuierliche Übertragung politischer Kompetenzen an die Europäische Union (EU) zu beobachten, mit der Folge, dass heute ca. $30 \%$ der vom Deutschen Bundestag verabschiedeten Gesetze auf einen "europäischen Impuls« (Töller 2008: 9) zurückgehen (vgl. König und Mäder 2008; Töller 2014; Tömmel 2014). Zugleich können Bürgerinnen und Bürger europäische Politik nicht nur über die nationale Ebene mitgestalten, sondern beispielsweise auch durch die Beteiligung an den Europawahlen oder an einer europäischen Bürgerinitiative (vgl. Oberle 2012; Beichelt 2009).

Diesem Wandel muss eine zeitgemäße politische Bildung Rechnung tragen (vgl. Oberle 2012; Oberle und Forstmann 2015a; Schöne und Immerfall 2015; Rappenglück 2014). Da nur die schulische politische Bildung sämtliche angehenden Bürgerinnen und Bürger erreichen kann, trägt sie für die politische Europabildung eine besondere Verantwortung. Bei der Vermittlung der Europäischen Integration haben Politiklehrkräfte auf Grund der hohen Dynamik und Komplexität der EU einen besonderen Bedarf nach Leitmedien (vgl. Detjen 2004; Oberle und Forstmann 2015b). Zugleich ergeben sich für Schulbücher des politischen Fachunterrichts angesichts dieser Dynamik und Komplexität, der empfundenen Bürgerferne der EU 
sowie verbreiteter Vorurteile bzw. Fehlkonzepte besondere Schwierigkeiten und Herausforderungen.

Schulischer Politikunterricht sollte Europa dabei sowohl als themenübergreifendes Unterrichtsprinzip als auch in einem speziellen Block zur Europäischen Integration mit Fokus auf die EU behandeln (vgl. Detjen 2004; Weißeno 2004). Dieses bildungspolitische Ziel lässt sich aus Forderungen der Europäischen Gemeinschaften bzw. der Europäischen Union und des Europarats (vgl. z. B. Mickel 1993; European Parliament Committee on Culture and Education 2016) ableiten und wurde ebenso seitens der Kultusministerkonferenz (KMK) formuliert sowie wiederholt bekräftigt (siehe z.B. KMK 2008). Insbesondere die Neufassung der Empfehlungen "Europa im Unterricht" von 1990 führte zu einer "Europäisierung" der nachfolgend novellierten Lehr- und Bildungspläne sowie der Schulbücher der Länder. Mittlerweile ist die Europäische Integration Bestandteil der Bildungspläne bzw. Kerncurricula für den politischen Fachunterricht in allen deutschen Bundesländern (vgl. Geyr et al. 2007). Die Vermittlung von Kompetenzen hinsichtlich der Europäischen Integration mit Schwerpunkt auf der Europäischen Union ist dabei insbesondere in den Sekundarstufen vorgesehen, in Niedersachsen beispielsweise im politischen Fachunterricht der 9. Klasse Hauptschule, 9./10. Klasse Realschule, 10. Klasse Gymnasium und 11. Klasse Integrative Gesamtschule (vgl. Niedersächsisches Kultusministerium, o. J.).

Die Bedeutung der Lehrpläne für die tatsächlich realisierte schulische politische Bildung muss allerdings relativiert werden: "Die geschriebenen sind nicht identisch mit den im Unterricht realisierten Lehrplänen." (Grammes 2004: 107) Was hier zum Thema EU passiert, liegt großteils noch immer im wissenschaftlich kaum beleuchteten Dunkeln. Wie Trommer (1999: 101) konstatiert: "Unterrichtsanalyen liegen nur vereinzelt vor. Es mag an der Randstellung des Fachs liegen, dass das Unterrichtsgeschehen hier seltener beschrieben wird als in anderen Fächern, zum Beispiel den Naturwissenschaften.« Eine Hilfestellung bei der empirischen Erforschung des tatsächlichen Unterrichtsgeschehens sind Schulbücher, da sie in den meisten Fächern noch immer als das Leitmedium der Unterrichtsgestaltung und v. a. der Inhaltsauswahl der Lehrenden gelten (vgl. Detjen 2001, 2005; Harms und Breit 1990). Ob Schulbücher allerdings tatsächlich (noch) als »eigentliche Großmacht der Schule» (Deutscher Bildungsrat 1969, zit. in 
Doll und Rehfinger 2012: 20) zu bezeichnen sind und welche Rolle dieses "Politicum «, "Informatorium « und »Pädagogicum» (Stein 1977, 1979; vgl. auch Höhne 2003) bei der politischen EU-Vermittlung im Schulunterricht spielt, sind empirisch unzureichend geklärte Fragen. So gibt es keine umfassende Übersicht darüber, welche der zugelassenen Politikbücher in Schulen vorhanden sind und ob und wie sie im politischen Fachunterricht eingesetzt werden. Auch die Aufbereitung der EU-Thematik in zugelassenen Schulbüchern des Politikunterrichts ist, anders als die Vermittlung der Europäischen Einigung in Geschichtsbüchern, empirisch kaum erforscht.

Die Brisanz des gewählten thematischen Fokus' der Schulbuchstudie auf die politische Europabildung wird deutlich, wenn man sich die besonderen Herausforderungen und Schwierigkeiten der spezifischen EU-Didaktik vergegenwärtigt, die Schulbücher für Lehrkräfte einerseits besonders relevant machen, andererseits aber zugleich deren Eignung für die Planung und Gestaltung des Politikunterrichts zur EU gefährden (vgl. Detjen 2004; Rappenglück 2004; Oberle und Forstmann 2015b): Erstens bedeutet die organisatorisch-institutionelle Komplexität der EU eine Hürde für den Erwerb korrekter EU-Kenntnisse. Das gilt insbesondere für ihre Entscheidungsverfahren und Kompetenzordnung, aber auch ihre verwirrenden Begrifflichkeiten. Die mangelnde Übertragbarkeit nationaler politischer Systemspezifika und Prozesse auf die EU erschwert zweitens ebenfalls das Verständnis. Die Komplexität der EU kann nicht "nebenbei« erschlossen werden - vielmehr muss eine Art kognitive politische Landkarte der EU in Form von Wissensnetzen und semantischen Strukturen erarbeitet werden, deren Elaboriertheit - Umfang, Verknüpfungsdichte, Relevanz und Korrektheit - entscheidend für die Anwendung (Transfer) und die Erweiterung (Lernen von Neuem) von Wissen ist (vgl. Kunter und Trautwein 2013; Seel 2003). Es bedarf daher einer didaktisch in besonderer Weise aufgearbeiteten, in angemessenem Maß komplexitätsreduzierten, an Präkonzepte und Vorurteile anknüpfenden, mit hilfreichen und korrekten Vergleichen und Bildern versehenen Aufbereitung des Themas in den entsprechenden Schulbüchern. Hinzu kommt drittens die hohe Dynamik des europäischen Integrationsprojekts, bei dem sich Bezeichnung, Mitglieder, Institutionen, Verfahren und Kompetenzen seit seiner Gründung alle paar Jahre geändert haben. Diese Dynamik bedeutet zugleich eine geringe Halbwertszeit des EUWissens, wovon auch Schulbücher betroffen sind. 
Punktuelle Studien (siehe z.B. Janssen 1995) haben festgestellt, dass Schulbuchdarstellungen der Europäischen Integration teilweise Fehlkonzepte generieren können und Schaubilder, Fakten und Zahlen nicht selten veraltet sind. Natterer (2001) untersuchte beispielsweise Politikschulbücher baden-württembergischer Haupt- und Realschulen und stellte fest, dass bezogen auf die Mitwirkungsrechte des Europäischen Parlamentes deren Entwicklungsdynamik oftmals ausgespart blieb, wobei konkrete Beispiele zum Gesetzgebungsverfahren meist fehlten und trotz bereits gegebener Aktualität des Mitentscheidungsverfahrens noch auf das Verfahren der Zusammenarbeit abgestellt wurde. Dies kann als Hinweis auf ein mehrfaches Defizit von Schulbüchern gewertet werden: erstens eine nicht ausreichende Thematisierung der Dynamik der EU, zweitens eine mangelnde Aktualität bei der Darstellung ihrer Entscheidungsmechanismen und drittens eine Unterbelichtung der Relevanz der Rolle des Europäischen Parlamentes. Natterer untersuchte in ihrer Dissertation darüber hinaus die sachliche Richtigkeit der Schulbuchdarstellungen und stieß dabei auf Defizite: zum einen auf ungenaue, die Leserinnen und Leser irreführende Darstellungen, die bei den Lernenden ein »falsches Bild « (Natterer 2001: 156) entstehen ließen, zum anderen auf fachlich falsche Darstellungen z.B. der Funktion des Europäischen Parlamentes in der Gesetzgebung, die unterschätzt oder widersprüchlich dargestellt wurde bzw. nicht dem jeweils aktuellen Stand entsprach. Auch bei der Anzahl der Mitgliedsstaaten und Angaben zu den Europawahlen fanden sich in den Schulbüchern Fehler bzw. lieferten sie veraltete Informationen.

Dazu kommt, dass die sprichwörtliche Bürgerferne der EU eine besondere Herausforderung für die politische Bildung darstellt, der Schulbücher Rechnung tragen müssen: Die Relevanz für die Lebenswelt der Schülerinnen und Schüler, ihren Alltag, ihre Interessen und ihre Zukunftspläne sollte mit geeigneten Beispielen herausgearbeitet werden. Auch sind politische Partizipationsmöglichkeiten der Bürgerinnen und Bürger in der EU zu thematisieren, wobei zugleich auf deren Grenzen eingegangen werden sollte - eine EU-Propaganda ist für die politische Bildung in Deutschland nicht angemessen und sollte im Sinne des Kontroversitätsprinzips und des Überwältigungsverbotes (Beutelsbacher Konsens, Wehling 1977; vgl. Oberle 2016) vermieden werden (vgl. auch Massing 2004; Sander 2004). Das gilt besonders für brisante Themen wie die Eurokrise, aber auch die Außen- und 
Sicherheits-, Migrations- und Agrarpolitik, bei denen unterschiedliche wissenschaftliche und politische Ansichten zu berücksichtigen sind.

Zusammenfassend lässt sich feststellen, dass Lehrkräfte bei der Vermittlung der Europäischen Integration und Europäischen Union im Politikunterricht einen besonderen Bedarf an Leitmedien haben, dass sich zugleich für Schulbücher aber bei diesem Thema u.a. angesichts der hohen Dynamik der Europäischen Integration, der großen Komplexität der EU, ihrer empfundenen Bürgerferne sowie verbreiteten Vorurteilen bzw. Fehlkonzepten besondere Schwierigkeiten und Herausforderungen ergeben, die bislang nicht zufriedenstellend bewältigt worden sind. Während die Darstellung der rezenten Europäischen Geschichte sowie von Europawahrnehmungen (Stichwort: Europäische Identität) in Geschichtsbüchern schon vergleichsweise gut erforscht ist (siehe z.B. das GEI-Projekt EurViews; Fuchs und Lässig 2009), bilden breit angelegte wissenschaftliche Untersuchungen zur Darstellung der Europäischen Integration in Schulbüchern des politischen Fachunterrichts und Analysen der tatsächlichen Verwendung des Schulbuchs bei der Planung und Gestaltung des Politikunterrichts zur EU wie auch Untersuchungen der Qualität der EU-Vermittlung aus Sicht der zentralen Akteure der Lehr-Lern-Prozesse - der Lehrkräfte und Lernenden - dringende Forschungsdesiderata. Diesem Forschungsbedarf hat sich das hier vorgestellte und durch das Ministerium für Wissenschaft und Kultur (MWK) Niedersachsen im Programm PRO^Niedersachsen geförderte Forschungsprojekt »Schulbücher als Vermittler der Europäischen Integration? Eine Studie zum politischen Fachunterricht« gestellt.

Die empirische Studie will einen Beitrag dazu leisten, die dargestellten eklatanten Forschungslücken bezüglich der Rolle von Schulbüchern bei der Vermittlung der EU im politischen Fachunterricht zu schließen. Basierend auf ausgewählten quantitativen und qualitativen Kriterien (zur Entwicklung von Rastern der Schulbuchanalyse vgl. z. B. Astleitner, Sams und Thonhauser 1998; Bamberger 1995; European Educational Publishers Group; Fritzsche 1995; Hilligen 1981; Laubig, Peters und Weinbrenner 1986; Schiller 1998; sowie die in quantitativen Studien entwickelten Codierungssysteme von Bromley, Meyer und Ramirez 2011a, 2011b) wurde hierfür erstens die Vermittlung der Europäischen Union und Europäischen Integration in Politikschulbüchern analysiert, die in den 16 Bundesländern von 2003 (Zäsur: Inkrafttreten des Vertrages von Nizza) bis 2012 für die 
Sekundarstufen allgemeinbildender Schulen erschienen sind. Dabei wurden auch die während des Untersuchungszeitraums eingetretenen Veränderungen der institutionellen Grundlagen der EU (insbesondere durch das Inkrafttreten des Vertrages von Lissabon 2009) berücksichtigt. Zweitens wurde die tatsächliche Verwendung dieser Bücher durch niedersächsische Politiklehrkräfte in Gymnasien und Gesamtschulen untersucht und dabei den Fragen nachgegangen, welche Bücher in den untersuchten Schulen verwendet werden, ob diese Bücher den Politiklehrkräften tatsächlich wie oftmals behauptet als Leitmedium (oder eher als didaktische Leitlinie oder Ergänzungsmedium, vgl. Typisierung von Thöneböhn 1995) bei der EUVermittlung dienen, welche Chancen und Defizite die Lehrkräfte an den Schulbuchdarstellungen ausmachen und welche weiteren Materialien und Medien gegebenenfalls als Ersatz oder Ergänzung Verwendung finden. Drittens schließlich wurden Klassen bzw. Kurse der befragten Lehrkräfte sowie Schülerinnen und Schüler weiterer niedersächsischer Gymnasien und Gesamtschulen, die das Thema EU vor Kurzem im Politikunterricht behandelt hatten, zur EU-Einheit des dabei verwendeten Schulbuchs befragt (in der Tat kam in allen untersuchten Klassen bei der EU-Behandlung ein Schulbuch zum Einsatz, obwohl dies kein Selektionskriterium darstellte).

Das Forschungsprojekt verbindet damit auf innovative Weise produktorientierte Inhaltsanalysen mit einer prozess- und wirkungsorientierten Schulbuchforschung, deren bisherige Vernachlässigung in der Schulbuchforschung vielfach beklagt wurde (vgl. z. B. Doll und Rehfinger 2012; Fuchs 2011). Diese im Projekt verfolgte Kombination eines produkt-, prozessund wirkungsorientierten Forschungsansatzes (vgl. Weinbrenner 1995) verspricht neuartige und wertvolle Einsichten. Chancen und Defizite von für die Sekundarstufen zugelassenen Politikschulbüchern hinsichtlich des Europathemas werden so offengelegt und Vorschläge für eine angemessenere Vermittlung der Europäischen Integration in künftigen Schulbüchern des politischen Fachunterrichts formuliert, mit dem Ziel, den Nutzen der Bücher für die Lehrerinnen und Lehrer und die Lernenden zu erhöhen. ${ }^{1}$

1 Wir danken Ellen Faust und Franziska Paraknewitz, Wissenschaftliche Hilfskräfte am Lehrstuhl Politikwissenschaft/Didaktik der Politik der Universität Göttingen, für ihre sehr engagierte und sorgfältige Mitwirkung an der Manuskripterstellung sowie Dr. Wibke Westermeyer vom Georg-Eckert-Institut für internationale Schulbuchforschung für ihre großartige Unterstützung im gesamten Lektorats- und Publikationsprozess. 


\section{Literatur}

Astleitner, Hermann, Jörg Sams und Josef Thonhauser. Womit werden wir in Zukunft lernen? Schulbuch und CD-Rom als Unterrichtsmaterialien. Ein kritischer Vergleich, Wien: Österreichischer Bundesverlag, 1998.

Bamberger, Richard. "Methoden und Ergebnisse der internationalen Schulbuchforschung im Überblick», in: Schulbuchforschung, Richard Olechowski (Hg.), Frankfurt/M.: Peter Lang, 1995, 46-94.

Beichelt, Timm. Deutschland und Europa. Die Europäisierung des politischen Systems, Wiesbaden: VS Verlag für Sozialwissenschaften, 2009.

Bromley, Patricia, John W. Meyer und Francisco Ramirez. »Student-Centeredness in Social Science Textbooks, 1970-2008: A Cross-National Study«, in: Social Forces 90 (2), 2011a, 547-570.

Dies. »The Worldwide Spread of Environmental Discourse in Social Studies, History, and Civics Textbooks, 1970-2008«, in: Comparative Education Review 55 (4), 2011b, 517-555.

Detjen, Joachim. "Schulbuchdidaktik. Anmerkungen zu Produktion, Rezeption und Didaktik von Schulbüchern zum Politikunterricht», in: Bildung und Erziehung 54, 2001, 459-482.

Ders. "Europäische Unübersichtlichkeiten «, in: Europa verstehen lernen. Eine Aufgabe des Politikunterrichts, Georg Weißeno (Hg.), Schwalbach/Ts.: Wochenschau Verlag, 2004, 126-143.

Ders. »Politikunterricht mit dem Schulbuch? Zustimmende, aber auch skeptische Gedanken über ein klassisches Unterrichtsmedium«, in: Politik unterrichten 1, 2005, 13-21.

Doll, Jörg und Anna Rehfinger. »Historische Forschungsstränge der Schulbuchforschung und aktuelle Beispiele empirischer Schulbuchwirkungsforschung", in: Schulbücher im Fokus. Nutzungen, Wirkungen und Evaluation, Jörg Doll, Keno Frank, Detlef Fickermann und Kurt Schwippert (Hg.), Münster/New York: Waxmann, 2012, 19-42.

European Educational Publishers Group. »Best European Schoolbook Award. Evaluation criteria", Schoolbook Awards, http://www.schoolbookawards.org/index. php?id=3\&language=uk, o. J., zuletzt geprüft am 26. August 2017.

European Parliament Committee on Culture and Education. »Report on Learning EU at school", https://www.europarl.europa.eu/doceo/document/A-8-2016-0021_ EN.html, 2016, zuletzt geprüft am 30. August 2020.

Fuchs, Eckhardt. »Aktuelle Entwicklungen der schulbuchbezogenen Forschung in Europa«, in: Bildung und Erziehung 64 (1), 2011, 7-22.

Fuchs, Eckhardt, Joachim Kahlert und Uwe Sandfuchs (Hg.). Schulbuch konkret. Kontexte, Produktion und Unterricht, Bad Heilbrunn: Klinkhardt, 2010. 
Fuchs, Eckhardt und Simone Lässig. "Europa im Schulbuch «, in: Geschichte für heute. Zeitschrift für historisch-politische Bildung 1, 2009, 60-66.

Fritzsche, Karl-Peter. »Europa in deutschen Politiklehrbüchern«, in: Macht Europa Schule? Die Darstellung Europas in Schulbüchern der Europäischen Gemeinschaft, Falk Pingel (Hg.), Frankfurt/M.: Diesterweg, 1995, 81-94.

Geyr, Maja von, Lilly Hornung, Friederieke Noack, Jaroslav Sonka und Eckart Stratenschulte. »Die Europäische Dimension in den Lehrplänen der deutschen Bundesländer: Vergleichende Studie im Auftrag der Europäischen Kommission", Europäische Kommission, http://ec.europa.eu/deutschland/pdf/work_study/ eab_studie.pdf, 2007, zuletzt geprüft am 26. August 2017.

Grammes, Tilman. »Architektoniken des Lehrplans - Welche Inhalte umfasst die politische Bildung?«, in: Demokratie braucht politische Bildung, Gotthard Breit und Siegfried Schiele (Hg.), Schwalbach/Ts.: Wochenschau Verlag, 2004, 99-116.

Harms, Hermann und Gotthard Breit. »Zur Situation des Unterrichtsfaches Sozialkunde/Politik und der Didaktik des politischen Unterrichts aus der Sicht von Sozialkundelehrerinnen und -lehrern. Eine Bestandsaufnahme«, in: Zur Theorie und Praxis der politischen Bildung, Bundeszentrale für politische Bildung (Hg.), Bonn: Bundeszentrale für politische Bildung, 1990, 13-167.

Hilligen, Wolfgang. "Kriterien für die Beurteilung von Lehr- und Lernmitteln für den politischen Unterricht in der Sekundarstufe», in: Historisch-politischer Unterricht, 4. Aufl., Hans Süssmuth (Hg.), Göttingen: Klett, 1981.

Höhne, Thomas. Schulbuchwissen: Umrisse einer Wissens- und Medientheorie des Schulbuchs, Frankfurt/M.: Universität Frankfurt/Fachbereich Erziehungswissenschaften, 2003.

Janssen, Bernd (Hg.). Die europäische Dimension in Lehr- und Lernmitteln. Bericht von der dritten Tagung zur europäischen Dimension in Unterricht und Erziehung, Bonn: Europa-Union-Verlag, 1995.

KMK. »Europabildung in der Schule - Beschluss der Kultusministerkonferenz vom 08.06.1978 i.d.F. vom 05.05.2008«, https://www.kmk.org/themen/allgemeinbil dende-schulen/weitere-unterrichtsinhalte/europabildung.html, zuletzt geprüft am 06. September 2020.

König, Thomas und Lars Mäder. "Das Regieren jenseits des Nationalstaates und der Mythos einer 80-Prozent-Europäisierung in Deutschland«, in: Politische Vierteljahresschrift 49 (3), 2008, 438-463.

Kunter, Mareike und Ulrich Trautwein. Psychologie des Unterrichts, Paderborn: Schöningh, 2013.

Laubig, Manfred, Heidrun Peters und Peter Weinbrenner. Methodenprobleme der Schulbuchanalyse, Bielefeld: Universität Bielefeld/Fakultät für Soziologie, 1986. Massing, Peter. »Bürgerleitbilder - Anknüpfungspunkte für eine europazentrierte Didaktik des Politikunterrichts«, in: Europa verstehen lernen. Eine Aufgabe des 
Politikunterrichts, Georg Weißeno (Hg.), Schwalbach/Ts.: Wochenschau Verlag, 2004, 144-157.

Mickel, Wolfgang W. Lernfeld Europa: Didaktische Grundlagen einer europäischen Erziehung, Opladen: Leske + Budrich, 1993.

Natterer, Alexandra. Europa im Schulbuch: Die Darstellung der europäischen Einigung in baden-württembergischen Schulbüchern für Geschichte und Gemeinschaftskunde der Sekundarstufe I, Grevenbroich: Omnia, 2001.

Niedersächsisches Kultusministerium. "Lehrpläne, allgemein bildende Schulen«, http://www.mk.niedersachsen.de/portal/live.php?navigation_id=1966\&article_ $\mathrm{id}=6378 \&$ \&.psmand=8, o. J., zuletzt geprüft am 06. September 2020.

Oberle, Monika. Politisches Wissen über die Europäische Union. Subjektive und objektive Politikkenntnisse von Jugendlichen, Wiesbaden: Springer VS, 2012.

Dies. »Der Beutelsbacher Konsens - eine kritische Würdigung", in: Brauchen wir den Beutelsbacher Konsens? Eine Debatte der politischen Bildung, Benedikt Widmaier und Peter Zorn (Hg.), Bonn: Bundeszentrale für politische Bildung, 2016, 251-259.

Oberle, Monika und Johanna Forstmann. »Förderung EU-bezogener Kompetenzen bei Schüler/innen - zum Einfluss des politischen Fachunterrichts«, in: Die Europäische Union erfolgreich vermitteln. Perspektiven der politischen EU-Bildung heute, Monika Oberle (Hg.), Wiesbaden: Springer VS, 2015a, 81-98.

Dies. "Lehrerfortbildungen zur politischen EU-Bildung - eine empirische Begleitstudie«, in: Die Europäische Union erfolgreich vermitteln. Perspektiven der politischen EU-Bildung heute, Monika Oberle (Hg.), Wiesbaden: Springer VS, 2015b, 193-209.

Rappenglück, Stefan. Europäische Komplexität verstehen lernen, Schwalbach/Ts.: Wochenschau Verlag, 2004.

Ders. »Europabezogenes Lernen«, in: Handbuch politische Bildung, 4. Aufl., Wolfgang Sander (Hg.), Schwalbach/Ts.: Wochenschau Verlag, 2014, 392-400.

Sander, Wolfgang. "Europa denken lernen«, in: Europa verstehen lernen. Eine Aufgabe des Politikunterrichts, Georg Weißeno (Hg.), Schwalbach/Ts.: Wochenschau Verlag, 2004, 158-171.

Seel, Norbert. Psychologie des Lernens, 2. Aufl., München: Reinhardt, 2003.

Schiller, Günter. »Methoden der Evaluation von Schulbüchern in Wirtschafts- und Arbeitslehre«, in: Erziehungswissenschaften und Beruf 46 (4), 1998, 427-438.

Schöne, Helmar und Stefan Immerfall. »EU-Bildung in der Schule - Erfahrungen und Desiderate«, in: Die Europäische Union erfolgreich vermitteln. Perspektiven der politischen EU-Bildung heute, Monika Oberle (Hg.), Wiesbaden: Springer VS, 2015, 67-80.

Stein, Gerd. Schulbuchwissen, Politik und Pädagogik. Untersuchungen zu einer praxisbezogenen und theoriegeleiteten Schulbuchforschung, Kastellaun: Henn, 1977. 
Ders. »Das Schulbuch im Spannungsfeld von pädagogischem Zweck, verlegerischer Investition und öffentlicher Kontrolle«, in: Schulbuch und Politik, Bernard Tewes (Hg.), Paderborn: Schöningh, 1979, 25-50.

Thöneböhn, Franz Heinrich. Rezeption und Verwendung des geographischen Schulbuchs in der Sekundarstufe I. Bochum: Universität Bochum, 1995.

Töller, Annette Elisabeth. »Mythen und Methoden. Zur Messung der Europäisierung der Gesetzgebung des Deutschen Bundestages jenseits des 80-Prozent-Mythos", in: Zeitschrift für Parlamentsfragen 39 (1), 2008, 3-17, https://doi.org/10.5771/ 0340-1758-2008-1-3, 2008, zuletzt geprüft am 06. September 2020.

Dies. »Europäisierung der deutschen Gesetzgebung. Wissenschaftliches Kurzgutachten «, https://ub-deposit.fernuni-hagen.de/servlets/MCRFileNodeServlet/mir_ derivate_00000959/T\%C3\%B61ler_Kurzgutachten_Europ\%C3\%A4isierung_Ge setzgebung_2014.pdf, 2014, zuletzt geprüft am 30. August 2020.

Tömmel, Ingeborg. Das politische System der EU, 4. Aufl., München/Wien: Oldenbourg, 2014.

Trommer, Luitgard. »Eine Analyse der Lehrpläne zur Sozialkunde in der Sekundarstufe I", in: Aufgaben politischer Bildung in der Sekundarstufe I: Studien aus dem Projekt Civic Education, Christa Händle, Detlev Oesterreich und Luitgard Trommer (Hg.), Opladen: Leske + Budrich, 1999, 69-129.

Wehling, Hans-Georg. »Konsens à la Beutelsbach? Nachlese zu einem Expertengespräch", in: Das Konsensproblem in der politischen Bildung, Siegfried Schiele und Herbert Schneider (Hg.), Stuttgart: Klett, 1977, 173-184.

Weinbrenner, Peter. "Grundlagen und Methodenprobleme sozialwissenschaftlicher Schulbuchforschung", in: Schulbuchforschung, Richard Olechowski (Hg.), Frankfurt/M.: Peter Lang, 1995, 21-45.

Weißeno, Georg. »Konturen einer europazentrierten Politikdidaktik - Europäische Zusammenhänge verstehen lernen", in: Europa verstehen lernen. Eine Aufgabe des Politikunterrichts, ders. (Hg.), Schwalbach/Ts.: Wochenschau Verlag, 2004, 108-125.

Wiater, Werner. Schulbuchforschung in Europa - Bestandsaufnahme und Zukunftsperspektive, Bad Heilbrunn: Klinkhardt, 2003.

Zürn, Michael. Regieren jenseits des Nationalstaates. Globalisierung und Denationalisierung als Chance, 2. Aufl., Frankfurt/M.: Suhrkamp, 2005.

Ders. A Theory of Global Governance. Authority, Legitimacy, and Contestation, Oxford: Oxford Univ. Press, 2018. 


\section{Die produktorientierte Teilstudie: Darstellung der Europäischen Union im Politikschulbuch}

Dieses Kapitel untersucht, wie die Europäische Union in deutschen Politikschulbüchern zwischen 2003 und 2012/13 dargestellt wird, wobei ein besonderer Fokus der gemischt-methodischen Analyse auf den in Niedersachsen zugelassenen Schulbüchern liegt. Die interdisziplinär angelegte Analyse verbindet Perspektiven der Politikwissenschaft, der Politikdidaktik und der Schulbuchforschung. Das Schulbuch als Untersuchungsgegenstand wird dabei nicht nur als didaktisches Mittel, sondern auch als kulturelles und politisches Medium verstanden. Schulbuchtexte stehen im Kontext gesellschaftlich-politischer Aushandlungsprozesse (vgl. Höhne 2003; Lässig 2009), ebenso wie die politische Bildung im Kontext gesellschaftlicher Entwicklungen steht (vgl. Eis 2010: 139).

Während es zahlreiche Studien gibt, die sich mit der Darstellung von Europa insbesondere in Geschichtsschulbüchern beschäftigen (vgl. Challand 2009; Karayianni 2011; Pingel 1995, 2009; Pingel und Boitsev 2000), mangelt es an systematischen Untersuchungen, die sich mit der Vermittlung des politischen Systems der EU in Politikschulbüchern befassen. Die letzte vergleichbare Studie von Natterer schließt Schulbücher für Geschichte und Gemeinschaftskunde in Baden-Württemberg bis zum Jahre 2001 ein, so dass direkt an diese Ergebnisse angeknüpft werden kann. Sowohl Natterer (2001: 264) als auch Mellies, Migdalski und Kühberger (2007) haben in ihren Untersuchungen festgestellt, dass die Europäische Integration in Schulbüchern oftmals als reibungsloser Prozess dargestellt wird und historische Krisen und Konflikte im Einigungsprozess teilweise ausgeblendet werden. Aus politikdidaktischer Perspektive haben Weißeno (2004) und Detjen (2004) kritisiert, dass die EU in den Schulbüchern oft als isoliertes Thema behandelt und aus rein nationaler 
Perspektive betrachtet wird. Eine weitere Herausforderung, die sich für die Darstellung der EU in Politikschulbüchern stellt, ist die Dynamik der Integration (besonders in den letzten Dekaden), durch die im Unterricht eingesetzte Schulbücher selten auf dem aktuellsten Stand sind und besondere Ansprüche an die Darstellung der Offenheit des Integrationsprozesses bestehen.

Die Studie möchte einen Beitrag leisten, die bestehende Forschungslücke in Bezug auf die Darstellung der EU in deutschen Politikschulbüchern zu schließen. Dabei interessiert unter anderem, welche Inhalte in den Kapiteln zur EU überhaupt vorkommen, wie sich die Darstellung bestimmter Aspekte zwischen Verlagen und Schulformen unterscheidet und wie sich diese im Zeitverlauf verändert. Im Sinne der kontextualisierenden Schulbuchforschung ist der gewählte Untersuchungszeitraum eine entscheidende Größe bei der Betrachtung des Gegenstandes Schulbuch (vgl. Mahamud 2015). Der Zeitraum, in dem die untersuchten Schulbücher veröffentlicht wurden, war von kontroversen Debatten und phasenweiser Stagnation des europäischen Integrationsprozesses geprägt, sowohl durch die Ablehnung der Verfassung für Europa in den Volksabstimmungen in Frankreich und den Niederlanden (2005) als auch durch die Finanz-, Banken- und Schuldenkrise (ab 2008). Von besonderem Interesse ist daher, wie in den Schulbuchtexten mit derartigen politischen Transformationsprozessen, der Dynamik des Integrationsprozesses, der Komplexität des Mehrebenensystems und der Distanz zwischen "Brüssel« und den EU-Bürgerinnen und -Bürgern umgegangen wird.

Zunächst wird das Forschungsdesign der Studie erläutert, anschließend werden ausgewählte Ergebnisse der Untersuchung vorgestellt. Diese betreffen folgende Forschungsfragen: Welche Inhalte zur EU kommen in den Schulbüchern vor? Wie aktuell, offen und kontrovers wird der europäische Integrationsprozess dargestellt? Wie werden die politikdidaktischen Prinzipien Schülerorientierung und Lebensweltbezug in den Schulbüchern umgesetzt?

\section{II.1 Fallauswahl und Analysemethode}

Für die Untersuchtung wurden zunächst alle Schulbücher für den Politikunterricht ${ }^{2}$ identifiziert, die zwischen 2003 und 2012 in Deutschland neu

2 Politikunterricht wird hier und im Folgenden als Bezeichnung für das Leitfach der 
erschienen sind. Ergänzend wurden Politikschulbücher einbezogen, die 2013 für Niedersachen erschienen sind, um die Anschlussfähigkeit an die hier durchgeführte Nutzungs- und Wirkungsstudie (siehe Kapitel III in diesem Band) zu erhöhen. Insgesamt sind dies knapp 450 Bücher. Davon waren etwa 170 Bücher für den Politikunterricht in den verschiedenen Bundesländern der 9./10. Klassen zugelassen, ${ }^{3}$ die auch ein Kapitel zu Europa enthalten. ${ }^{4}$ Ausgehend von den Schulbüchern, die Europa nicht nur als geografischen Raum oder aus historischer Perspektive, sondern auch als politisch-wirtschaftliches System thematisieren, wurde für die Untersuchung eine Auswahl von 87 Schulbuchkapiteln aus ganz Deutschland getroffen (deutschlandweites Sample: DEUS), wobei 30 davon für Niedersachsen (bzw. Niedersachsen und weitere Bundesländer) zugelassen waren ${ }^{5}$ (niedersächsisches Sample: NIS). ${ }^{6}$ Bei der Auswahl spielten die Variablen Erscheinungsjahr, Verlag und Schulform eine wichtige Rolle, um die Grundgesamtheit möglichst angemessen zu repräsentieren und Vergleiche zwischen Verlagen und Schulformen und über die Zeit zu ermöglichen. In Bezug auf die Bundesländer sind alle Regionen (mit Zulassungsverfahren) abgedeckt, allerdings ist das DEUS-Sample zu Gunsten von Niedersachsen verzerrt, da aus diesem Bundesland sämtliche im Untersuchungszeitraum zugelassenen Politikschulbücher mit EU-Kapitel aufgenommen wurden, während aus den anderen Bundesländern jeweils eine Auswahl der Bücher getroffen wurde.

Dass einige Verlage mehr Neuausgaben einer Reihe auf den Markt bringen als andere und es zudem Jahre gibt, in denen es mehr Neuerscheinungen gibt als in anderen, wurde bei der Auswahl berücksichtigt. Im DEUS $(n=87)$

politischen Bildung an Schulen verwendet, dessen Benennung deutschlandweit zwischen den Bundesländern und verschiedenen Schulformen stark variiert (z.B. Gemeinschaftskunde, Sozialkunde, Sozialwissenschaften, Politik/Wirtschaft, Gesellschaftslehre; vgl. Oberle und Pohl 2020).

3 Ausnahme NRW, da hier die EU in der 8. Klasse unterrichtet wird.

4 Die Identifikation der relevanten Schulbücher war möglich durch eine Sichtung der bundesweiten Curricula (teils über die GEI-Plattform Curricula Workstation), der Listen der zugelassenen Schulbücher (teils über die GEI Homepage edumeres.net) und eine Sichtung der Inhaltsverzeichnisse (als PDF vorhanden im Bibliothekskatalog des GEI).

5 Berufsschulen ausgenommen.

6 Zur Samplezusammensetzung siehe Literaturangaben aller untersuchten Schulbücher auf S. 85-93. 


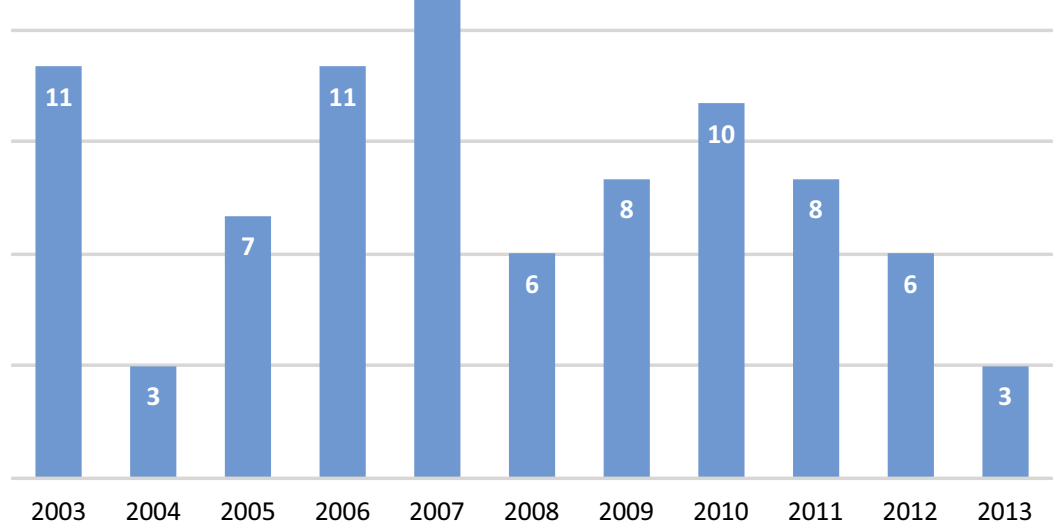

Abbildung 1: Verteilung der Erscheinungsjahre im Sample DEUS (Häufigkeiten)

stammen daher besonders viele Bücher aus den Jahren 2003 und 2006 (je 12,6 \%, elf Bücher) sowie 2007 (16,1\%, 14 Bücher), bei den anderen Jahrgängen liegt die Anzahl zwischen drei und zehn Büchern pro Jahr (3,4-11,5\%; vgl. Abbildung 1). Im NIS ( $n=30)$ gibt es kein Buch aus dem Jahr 2004, das im untersuchten Zeitraum zugelassen war. Die meisten Bücher stammen hier aus den Jahren 2007 (23,3\%, sieben Bücher) und 2010 (20\%, sechs Bücher), in den übrigen Jahren liegt die Zahl zwischen ein und drei neuerschienenen und in Niedersachsen zugelassenen Büchern pro Jahr (3,3-10 \%).

Im DEUS wurden alle Verlage, die in der Erhebung identifiziert wurden, in der Fallauswahl berücksichtigt (vgl. Abbildung 2). So wurde gewährleistet, dass auch kleine Verlage mit wenigen Neuauflagen oder Länderausgaben analysiert wurden. $\mathrm{Zu}$ den kleinen Verlagen, die mit ein bis zwei Büchern im DEUS vertreten sind, gehören Auer, Bildungsverlag Eins, Duden-Paetec, Militzke und Oldenbourg. Die größeren Verlage C. C. Buchner, Cornelsen, Klett, Schöningh und Westermann sind mit je neun bis dreizehn Büchern im DEUS abgebildet. Die weitaus höchste Anzahl an Ausgaben stellt jedoch - entsprechend der hohen Anzahl an Neuausgaben zwischen 2003 und 2012 - Schroedel mit 26 Büchern (29,9\%). In Niedersachsen waren, bis auf ein Buch von Duden-Paetec, keine Bücher aus den kleineren Verlagen zugelassen. Im NIS stammen die meisten zugelassenen Bücher 
von Klett, Schroedel und Westermann (je $20 \%$ ), gefolgt von Schöningh und Cornelsen (je 13,3\%) sowie C.C. Buchner (10 \%).

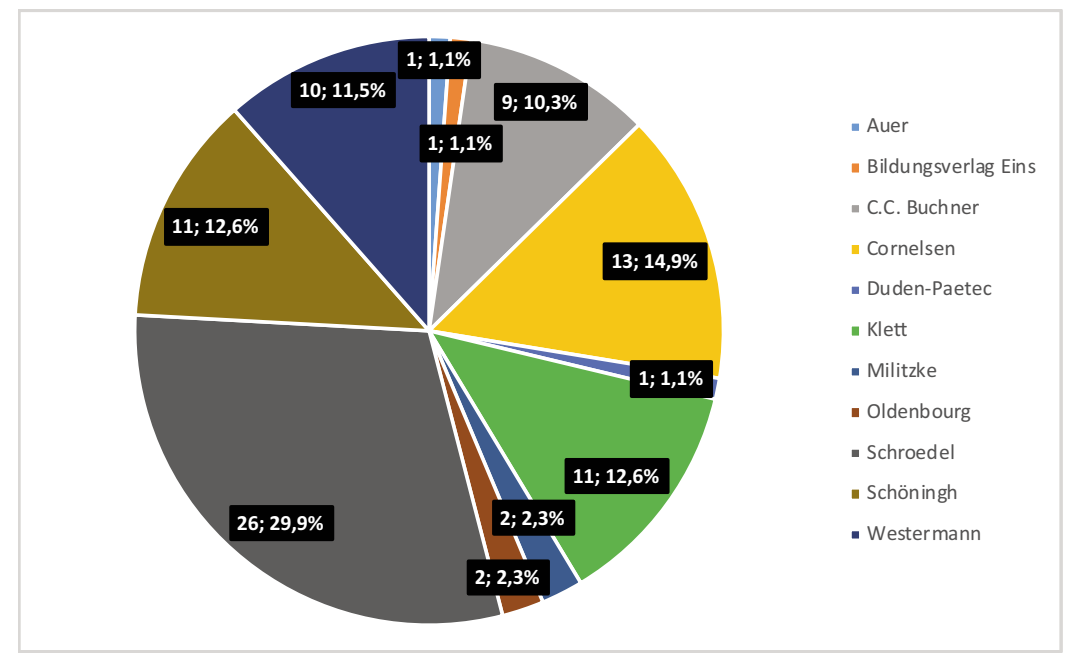

Abbildung 2: Verteilung der Verlage im Sample DEUS (Häufigkeiten und Prozentwerte)

Was die Schulformen betrifft, so wurden diese zur besseren Vergleichbarkeit teilweise in Gruppen zusammengefasst, in dem Bewusstsein, dass die Fülle an unterschiedlichen Schulformen in Deutschland so nur oberflächlich dargestellt wird. ${ }^{7}$ Die Zuordnung zu Schulform-Gruppen war auch deswegen notwendig, da ein Schulbuch in unterschiedlichen Bundesländern durchaus in verschiedenen Schulformen zugelassen sein kann. Interessanterweise gab es kaum Schulbücher, die ausschließlich für die Gesamtschule zugelassen waren - dies spiegelt sich insbesondere im NIS wider. Im DEUS waren die meisten Bücher ausschließlich für das Gymna-

7 Folgende Kategorien der Variable »Schulform» wurden gewählt:

- Hauptschule

- Realschule

- Gymnasium

- Gesamtschule

- Verschiedene Schulformen mit Gymnasium (VSmG)

- Verschiedene Schulformen ohne Gymnasium (VSoG)

- Ohne Angabe 
sium zugelassen (27 Bücher, $31 \%$ ). 18,4 \% der Bücher waren für die Realschule konzipiert (16 Bücher) und 12,6 \% für die Haupt- bzw. Mittelschule (elf Bücher). Weitere 30 Bücher (34,5\%) waren für verschiedene Schulformen zugelassen, zehn davon (11,5\%) auch für das Gymnasium. Im NIS waren ebenfalls die meisten Bücher ausschließlich für das Gymnasium zugelassen (elf Bücher, 36,7 \%), sechs Bücher (20\%) für die Realschule und zwei für die Hauptschule (6,7 \%). Bücher, die für verschiedene Schulformen ohne Gymnasium zugelassen waren, nehmen in Niedersachsen $20 \%$ ein (sechs Bücher), weitere 16,7 \% (fünf Bücher) waren zudem für VSmG zugelassen.

Zehn der 30 in Niedersachsen zugelassenen Schulbücher waren auch in anderen Bundesländern (z. B. Hamburg oder Bremen) zur Nutzung im Politikunterricht zugelassen. Abgesehen von der Verzerrung zu Gunsten von Niedersachsen sind Nordrhein-Westfalen (12,6\%), Bayern und BadenWürttemberg (mit Saarland) (je $8 \%$ ) sowie Hessen und Rheinland-Pfalz (mit Saarland) (je 6,9 \%) mit den meisten Länderausgaben im Sample vertreten. Die übrigen Bundesländer (mit Zulassungsverfahren) sind mit je ein bis vier Büchern (1,2-4,6 \%) im Sample vertreten. Zwei Ausgaben für Berlin wurden aufgenommen, auch wenn der Stadtstaat seit 2004 keine Zulassungspflicht mehr vorsieht und so keine Angaben zur Schulform gemacht werden können.

Als Untersuchungsmethode wurde ein gemischt-methodisches Vorgehen gewählt: Alle 87 Fälle (DEUS) wurden mit der Methode der automatisierten, wörterbuchbasierten, quantitativen Inhaltsanalyse im Sinne einer Breitenanalyse untersucht. Die 30 niedersächsischen Schulbücher (NIS) wurden zudem mit der Methode der qualitativ-strukturierenden Inhaltsanalyse nach Kuckartz (2014) zwecks Tiefenanalyse erforscht. Generell zeichnet sich die Methode der Inhaltsanalyse dadurch aus, dass sie sich von »der freien und spontanen Deutung « von Kommunikationsinhalten, wie sie im Alltag vorkommt, abgrenzt, durch eine Interpretation, die »systematisch, regelgeleitet und theoriebasiert erfolgt « (Lamnek 2000: 323). Während qualitative, inhaltsanalytische Verfahren in der Schulbuchforschung etabliert sind, sind quantitative Inhaltsanalysen, wie sie in dieser Studie zur Anwendung kommen, seltener vertreten (Schmit 2015: 53). Die Analyse des Materials erfolgte jeweils computergestützt (Software MAXQDA11), eine Vorgehensweise, die in der Schulbuchforschung bisher nicht stark vertreten 
ist, obwohl sie die Vorzüge der besseren Nachvollziehbarkeit der (qualitativen) Forschung und der leichten Überprüfbarkeit der (quantitativen) Ergebnisse bietet (Slopinski und Selck 2014: 130). Die Kombination von qualitativer und quantitativer Inhaltsanalyse hat den Vorteil, dass sie sich gegenseitig sinnvoll ergänzen können:

Quantitative Inhaltsanalysen reißen erfasste Textmerkmale zunächst aus ihrem Zusammenhang, um sie anschließend mittels Datenaggregation wieder zu rekontextualisieren. [...] Während quantitative Inhaltsanalysen zwangsläufig an der Textoberfläche bleiben (müssen), da sie sich hauptsächlich auf den propositionalen Gehalt des Textes beziehen, sind qualitative Analysen in der Lage, latente Kommunikationsinhalte zu erhellen. (Baros und Wilke 2015: 316)

In der vorliegenden Studie stellte die qualitative Inhaltsanalyse des Materials (NIS) den Startpunkt dar. Bei der inhaltlich-strukturierenden Inhaltsanalyse stehen thematische Kategorien im Mittelpunkt, mit denen für die Forschungsfragen relevante Inhalte im Material erfasst und anschließend interpretiert werden. Zu diesem Zweck wurden in der MAXQDAProjektdatei ein Kategorien- bzw. Codesystem angelegt, ein Codierleitfaden erstellt und das Material entsprechend analysiert (vgl. Mayring 2002: 119f.). Dabei wurde ein deduktiv-induktives Vorgehen bei der Kategorienentwicklung angewandt, welches den Vorteil bietet, offen für die Kategorienbildung aus dem Material heraus zu sein und gleichzeitig (durch die deduktive Herangehensweise) mögliche Leerstellen im Material offenlegen zu können. Das vollständige Kategoriensystem der qualitativen Untersuchung umfasst 32 Hauptkategorien (Codes) und 312 Unterkategorien (Subcodes). Dabei wurden inhaltlich-thematische Aspekte wie z. B. Politikbereiche, Institutionen und Verträge der EU, der historische Integrationsprozess und Gesetzgebungsprozesse, aber auch Alltags-, Regionalbezug und Schülerorientierung sowie strukturelle und didaktische Elemente wie Bilder (Fotos, Karten, Karikaturen, schematische Darstellungen etc.), Überschriften, Aufgaben, Methoden und Unterrichtsprinzipien erfasst. In der qualitativen Auswertung der codierten Textstellen wurden Fallanalysen und Vergleiche der Darstellungsweisen der Themen in Bezug auf Verlage, Schulformen und den Zeitpunkt der Veröffentlichung (vor oder nach dem Vertrag von Lissabon 2009) erstellt. 
Ausgehend von den Kategorien der qualitativen Analyse, wurde ein Schlagwortraster (Diktionär) für die wörterbuchbasierte quantitative Inhaltsanalyse entworfen, mit dem das gesamte Material (DEUS) analysiert und einer automatischen Codierung ${ }^{8}$ unterzogen wurde. ${ }^{9}$ Dieses Vorgehen lässt sich als »halbautomatische Inhaltsanalyse« klassifizieren, in der "parallel zur manuellen Inhaltsanalyse, welche sich auf die inhaltliche Bedeutung von Texten beschränkt, eine automatische Analyse [...], welche computerlesbare Eigenschaften derselben Texte [z. B. Länge der Texte oder das Vorkommen bestimmter Begriffe] erhebt«, durchgeführt wurde (Wettstein 2014: 18). Die automatische Codierung erfolgte mit Hilfe des Tools MAXdictio in MAXQDA11, die Auswertung im Anschluss mit der Software SPSS. Mithilfe deskriptiver Statistik und Häufigkeitsanalysen kann so veranschaulicht werden, wie sich die absoluten und relativen Häufigkeiten der thematischen Kategorien im Zeitverlauf verändern und ob es Unterschiede in der Häufigkeit in Bezug auf Verlage, Schulformen und Erscheinungsjahre gibt.

\section{II.2 Ergebnisse}

Im Folgenden werden die Ergebnisse der quantitativen und qualitativen Inhaltsanalysen gemeinsam anhand der identifizierten thematischen Kategorien vorgestellt. Der erste Abschnitt gibt einen kurzen Überblick über die zentralen Themen und Inhalte zur EU in den Politikschulbüchern, so die Häufigkeit thematisierter Politikbereiche und Organe sowie die Darstellung des Mehrebenensystems der EU. Der zweite Abschnitt beantwortet die Frage nach der Aktualität und Kontroversität der Schulbuchtexte. Der dritte Abschnitt geht auf die Aspekte »EU im Alltag«, Lebensweltbezug und Schülerorientierung ein.

8 Hier verstanden als die automatische Auszählung von Schlagworten, die einer thematischen Kategorie zugeordnet sind.

9 Begriffe mit Mehrfachbedeutungen müssen ausgeschlossen werden. Außerdem muss das Diktionär ans Material angepasst sein, dies kann beispielsweise bestimmte Fachbegriffe (z.B. aus einem Politikbereich) als Suchworte ausschließen, da sie im Schulbuchtext keine Treffer generieren (dies wurde in der Entwicklung der quantitativen Kategorien berücksichtigt). 
Nur EU-Institutionenkunde? Die Themen und Inhalte zur EU in den untersuchten Schulbüchern

Der Raum, den das Thema EU (und Europa) in den Politikschulbüchern einnimmt, ist in den vergangenen Jahren etwas größer geworden, wie der gestiegene Umfang der EU-Kapitel. So lag der durchschnittliche Umfang der EU-Kapitel zwischen 2003 und 2008 bei 29 Seiten und zwischen 2009 und 2012/13 bei 33 Seiten. Während das Minimum an Seitenzahlen eines EUKapitels zwischen 2003 und 2008 bei acht Seiten lag, waren es in der Zeit von 2009 bis 2012/13 bereits mindestens fünfzehn Seiten, auf denen die EU thematisiert wurde. Allerdings hat der Seitenumfang der Politikschulbücher auch generell im Untersuchungszeitraum zugenommen, von durchschnittlich 240 Seiten für den Zeitraum 2003 bis 2008 auf 273 Seiten für den Zeitraum von 2009 bis 2012/13. Das Maximum an Seitenzahlen erhöhte sich von 376 auf 549 Seiten, während der geringste Umfang in beiden Zeiträumen bei 128 Seiten lag.

Die folgenden Säulendiagramme (vgl. Abbildungen 3 und 4) zeigen die Mittelwerte der Anteile der thematischen Kategorien »Politikbereiche« und "EU-Organe« im Vergleich der Zeitperioden 2003 bis 2008 und 2009 bis 2012/13. Der Anteil der Kategorien bezieht sich dabei jeweils auf die Anzahl der Treffer (also gefundenen Schlagworte) einer Kategorie im Verhältnis zur Gesamtwörterzahl des jeweiligen Falles (Kapitels). Die Diagramme veranschaulichen, dass sich die Anteile der meisten Themenbereiche im Vergleich der Zeitabschnitte kaum verändern. Auffällig sind lediglich der Anstieg im Politikbereich »Wirtschaft/Finanzen/Steuern« und »Justiz/Inneres«, wie auch der Zuwachs beim Anteil »Europäische Zentralbank«. Empirisch lässt sich dies mit der Thematisierung der Finanz-, Banken- und Schuldenkrise der EU in den Schulbüchern sowie dem Aufleben der Debatte um die EU-Asyl- und Flüchtlingspolitik in den letzten Jahren erklären. Neben diesen inhaltlichen Veränderungen, die sich quantitativ nachweisen lassen, zeigt Abbildung 3 auch, dass nicht nur der Anteil aus dem Politikbereich »Beschäftigung/Soziales « gesunken ist, sondern dass der Anteil aus dem Politikbereich "Kultur/Bildung/Jugend" gleichbleibend gering den drittletzten Rang einnimmt. Zwar hat die EU in den Bereichen Kultur sowie Jugend, (allgemeine und berufliche) Bildung und Sport nur ergänzende und koordinierende Zuständigkeiten (Art. 6 AEUV, vgl. Europäische Union 
2010), dennoch weist dieses quantitative Ergebnis bereits auf die Vernachlässigung eines Themenbereiches in den Schulbüchern hin, über den ein Lebensweltbezug für die Schülerinnen und Schüler hergestellt werden könnte.

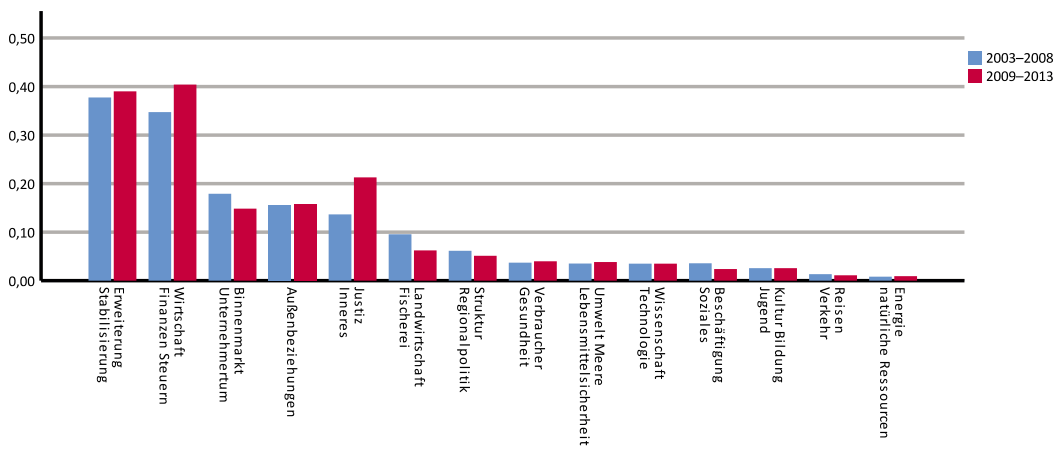

Abbildung 3: Anteile der Kategorien „Politikbereiche“ im Vergleich der Zeitperioden (Mittelwerte für 2003-2008 versus 2009-2013)

Das Säulendiagramm zu den Anteilen der EU-Organe (vgl. Abbildung 4) verdeutlicht, dass die Europäische Kommission etwa doppelt so häufig in den EU-Kapiteln vorkommt wie das Europäische Parlament und der Rat der EU - und dies, obwohl das EU-Parlament mit dem Vertrag von Lissabon (2009) an Kompetenzen hinzugewonnen hat, was eine stärkere Thematisierung des Organs in den Schulbüchern rechtfertigen würde. Dass es sich bei der Darstellung der EU in den Schulbüchern jedoch um reine »Institutionenkunde" handelt, wie etwa von Detjen (2004) und Weißeno (2004) kritisiert wurde, lässt sich mit einem Blick in die absoluten Häufigkeiten nicht unbedingt bestätigen. So finden sich zwischen 2003 und 2008 durchschnittlich 27,5 Schlagworte zur Kommission in den Kapiteln. Im selben Zeitraum sind es allein für den Bereich »Erweiterung und Stabilisierung" jedoch 39 Schlagworte. Zwischen 2009 und 2012/13 steigt der durchschnittliche Wert der absoluten Nennungen zur Kommission auf 35, beim Thema Erweiterung und Stabilisierung sind es dagegen 52 Schlagworte.

Relevant zu erwähnen ist an dieser Stelle, dass es vor 2009 noch Fälle gibt, in denen trotz der Bedeutung der Europäischen Kommission und des Europäischen Parlaments beide Institutionen gar nicht in den Kapiteln vor- 


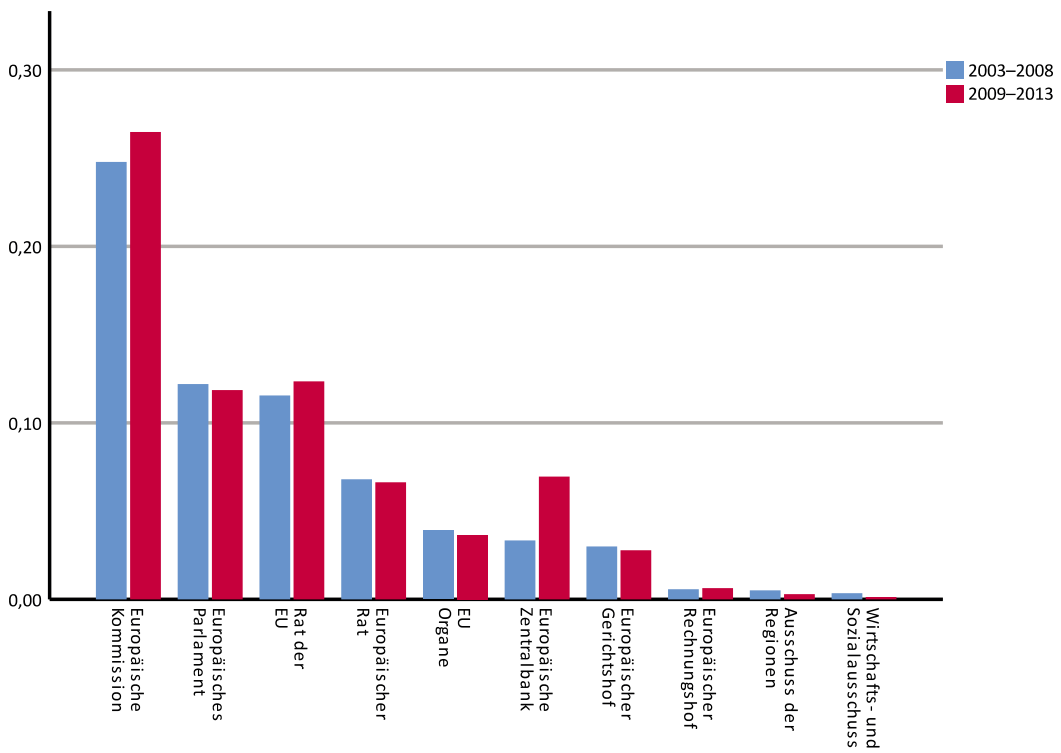

Abbildung 4: Anteile der Kategorie „EU-Organe“ im Vergleich der Zeitperioden (Mittelwerte für 2003-2008 versus 2009-2013)

kommen, während dies ab 2009 nicht mehr der Fall ist. Die qualitative Analyse zeigt außerdem, dass der Kompetenzzuwachs des Europäischen Parlaments im Rahmen des EU-Reformprozesses in den niedersächsischen Schulbüchern durchaus abgebildet wird, wenn auch in unterschiedlicher Tiefe (vgl. Bischewski 2020).

Nach diesem kurzen allgemeinen Überblick über die Themen in den EUKapiteln wird im Folgenden auf die Darstellung der EU als politisches System anhand konkreter Beispiele näher eingegangen. Dass die Komplexität des EU-Mehrebenensystems in den Politikschulbüchern unzureichend dargestellt würde, bemängeln bereits Weißeno (2004) und Detjen (2004). Diese Kritik lässt sich auch mit den Ergebnissen dieser Untersuchung nicht entkräften. Die Kategorie "Mehrebenensystem" wurde qualitativ und quantitativ analysiert. Mit der qualitativen Inhaltsanalyse nach Kuckartz (2014) wurden im NIS alle Abschnitte erfasst, die die EU als "System sui generis«, »Bundesstaat oder Staatenbund « erläutern oder das DemokratieDefizit der EU erwähnen. Die Schwierigkeit bei der Entwicklung dieser 
Kategorie bestand darin, dass der in der Politikwissenschaft etablierte Begriff »Mehrebenensystem» in den niedersächsischen Schulbüchern kaum genutzt wird, um das politische System der EU zu beschreiben. Tatsächlich kommt er nur in drei der 30 niedersächsischen Bücher vor, wobei er in zweien davon lediglich als Überschrift für eine schematische Darstellung fungiert und im Fließtext nicht weiter aufgegriffen wird. Bei diesen drei Büchern (Mensch und Politik 2007, Westermann; Politik-Wirtschaft 2007, Schöningh; Politik-Wirtschaft 2007, Duden-Paetec) handelt es sich auch nicht um die aktuellsten Bücher des Samples, sondern um Ausgaben von 2007. Dass es sich bei der EU um ein politisches System besonderer Art handelt, wird zwar in fast allen niedersächsischen Büchern erwähnt, eine Erläuterung oder Beschreibung der verschiedenen Ebenen erfolgt jedoch nicht. Die quantitative Analyse zeigt außerdem, dass eine Thematisierung des »Mehrebenensystems" (erfasst mit Begriffen wie "sui generis", »intergouvernemental « oder "supranational«) in den Schulbüchern für das Gymnasium am häufigsten vorkommt (gesamter Zeitraum $M=0,02$ ) und in den Schulbüchern, die für verschiedene Schulformen ohne das Gymnasium gedacht sind, am seltensten $(M=0,01)$.

Die unzureichende Erwähnung und Darstellung des Mehrebenensystems ist ein Beispiel für die Problematik, dass Fachterminologie in den untersuchten Politikschulbüchern kaum genutzt wird (zur Relevanz der Verwendung von Fachsprache im Politikunterricht vgl. Weißeno 2013). Vielmehr werden etablierte Begriffe oder Bezeichnungen umschrieben. Nur wenige Bücher nutzen die Möglichkeit eines Glossars oder der Definition von Fachbegriffen am Seitenrand, was bei der »verwirrenden Terminologie» der EU sicherlich eine ungenutzte Chance der verständlichen Vermittlung ist (Detjen 2004: 126). Dies gilt umso mehr, da Tatje (2017) in seiner Studie herausgefunden hat, dass gerade Schülerinnen und Schüler mit Migrationshintergrund die Politikschulbücher zum Nachschlagen benutzen.

Aktuell, offen und kontrovers? Der Integrationsprozess der EU

Der folgende Abschnitt widmet sich den Fragen, wie Veränderungen im Kontext der EU in den Schulbuchinhalten abgebildet werden und inwiefern die Dynamik der Integration ein Problem für die Aktualität der Schulbücher 
darstellt. Dabei interessiert auch die Vermittlung der Offenheit des Integrationsprozesses, der als nicht abgeschlossen gilt. Des Weiteren wird erläutert und problematisiert, wie der historische Prozess der Europäischen Integration dargestellt wird. Abschließend werden in diesem Abschnitt Vorurteile zur EU aufgegriffen, die in den Politikschulbüchern abgebildet werden.

Wie bereits im ersten Abschnitt der Ergebnisdarstellung gezeigt, lassen sich Veränderungen in den Anteilen und Häufigkeiten bestimmter Themen im Vergleich der Zeitperioden feststellen. Abbildung 5 zeigt als Liniendiagramm die Entwicklung der Kategorie "Wirtschaft/Finanzen/Steuern« im Zeitverlauf. Der Verlauf der Linie weist einen kontinuierlichen Anstieg ab dem Jahr 2007 auf. Es liegt nahe, dass dieser Anstieg auf die "Eurokrise» zurückzuführen ist.

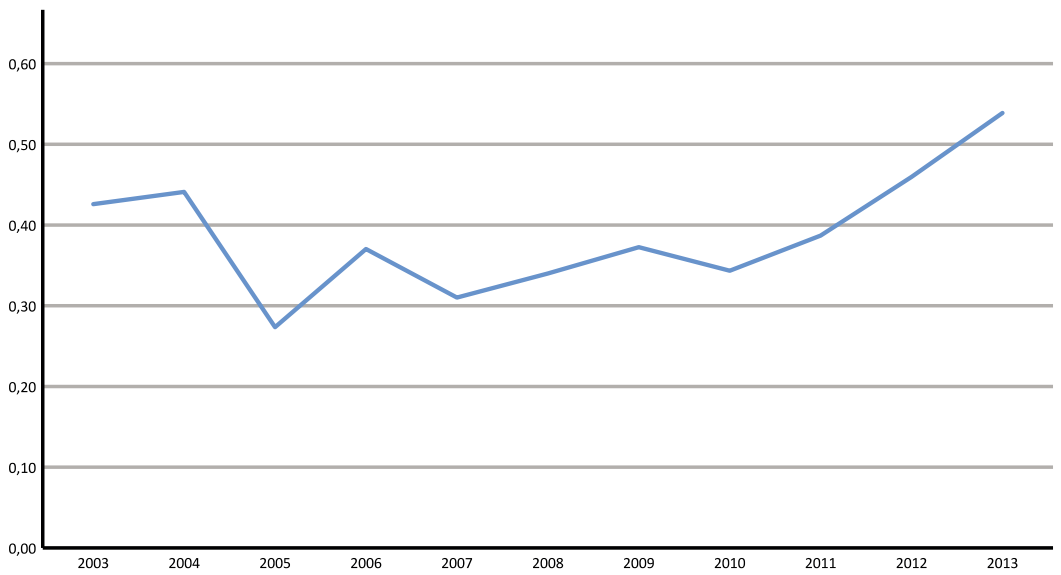

Abbildung 5: Entwicklung des Anteils der Kategorie „Wirtschaft/Finanzen/Steuern“ im Zeitverlauf (2003-2013)

Die quantitativen Auswertungen weisen darauf hin, dass Schulbuchinhalte rascher aktualisiert werden, als ihr Ruf vermuten lässt (»Kein Schulbuch veraltet so schnell wie ein Politikschulbuch«, Pohl 2014: 187). So werden den Ergebnissen der qualitativen und quantitativen Inhaltsanalyse zufolge beispielsweise wichtige Ereignisse auf europäischer Ebene - wie das Scheitern der geplanten Verfassung für Europa oder die Ratifizierung des 
Vertrages von Lissabon - rasch in die Schulbuchtexte integriert. Der Vertrag von Lissabon etwa wird bereits 2007 in vier Büchern thematisiert, ab 2009 (also nach der Ratifizierung) fehlen nur in vier Büchern Hinweise darauf. Die Mehrzahl der Bücher ist somit in Bezug auf die vertraglichen Änderungen durchaus auf dem aktuellen Stand.

Was die Umsetzung des Kontroversitätsprinzips und die Darstellung von Krisen und Dynamik der Europäischen Integration angeht, ergibt die Analyse kein eindeutiges Bild. Auf der einen Seite benutzen z. B. alle großen Verlage (mit Ausnahme von Cornelsen) in insgesamt 22 der 87 Ausgaben (zehn davon aus Niedersachsen) eine Karikatur, die Europa als überforderte Mutter darstellt, die jedem ihrer jammernden Kleinkinder (den Mitgliedstaaten) ein eigenes, landestypisches Gericht zubereiten soll. Mit dieser Karikatur wird auf das grundsätzliche Problem der Eigeninteressen der Mitgliedstaaten im Integrationsprozess hingewiesen, welche eine Herausforderung für die Gemeinschaft darstellen. Auf der anderen Seite zeigt insbesondere die qualitative Analyse, dass die Darstellung konkreter Hürden und der Offenheit der weiteren Integration bei der Formulierung der Schulbuchtexte teilweise missverständlich ist. Beispielsweise wird auf einer Seite eines Schulbuches festgestellt, dass es zukünftig eine Verfassung für Europa geben werde, wenige Seiten später wird jedoch auf das Scheitern der Verfassung verwiesen (Politik.21 2012: 15). Besonders problematisch ist ein Autorentext aus dem Jahr 2010, in dem auf irreführende Weise der Vertrag von Lissabon als Verfassung bezeichnet wird:

Der Vertrag von Lissabon (Verfassung) trat am 1. Dezember 2009 in Kraft. Allerdings sind sich die Mitgliedsstaaten uneins darüber, ob es in der Zukunft die "Vereinigten Staaten von Europa " geben soll. Die Verfassung darf deshalb offiziell nicht "Verfassung« heißen. Welche Zukunft hat Europa? (Anstöße 2 2010: 48)

Das Scheitern der Verfassung und die daraus resultierende Entwicklung des Vertrages von Lissabon stehen für die Stagnation und Sinnkrisen, die die EU in den vergangenen Jahrzehnten der politischen Integration immer wieder erlebt hat. Doch wie bereits von Natterer (2001) und Mellies, Migdalski und Kühberger (2007) kritisiert wurde, werden Konflikte im Integrationsprozess selten in den Schulbüchern thematisiert und verschwinden aus den Schulbüchern, je länger sie zurückliegen - so auch die Verfassungskrise (vgl. Bischewski 2020). Den Integrationsprozess der EU als reibungslos und 
nahezu konfliktfrei darzustellen, ist sicher einer notwendigen didaktischen Reduktion geschuldet, allerdings birgt dies die Gefahr, Abläufe »unterkomplex« darzustellen (Weißeno 2004: 120). Der historische Verlauf der Einigung wird beispielsweise in den Verlagen Cornelsen und Schroedel mit einer Bildmetapher in Form eines illustrierten Zeitstrahls veranschaulicht, was eine solche Wahrnehmung der europäischen Einigung als reibungsloser Prozess unterstützt. Hier werden (meist zu Beginn der Kapitel) die "Stationen auf dem Weg der Einigung" als Radtour (Cornelsen, in allen 13 Büchern) oder "Der Weg der Europäischen Union" als Wanderung (Schroedel, in 14 von 26 Büchern) dargestellt. Dabei sind die wichtigsten Integrationsschritte auf Wegweisern aufgeführt, die von einer Gruppe von Radfahrern bzw. Wanderern passiert werden. Die Gruppe der Personen wächst dabei im Verlauf des Integrationsprozesses/Weges an. Platz für Hinweise auf Krisen oder Uneinigkeiten bietet eine solch kontinuierlich anmutende Darstellung nicht. Auch aus der Darstellung des Gesetzgebungsprozesses gehen die Konflikthaftigkeit und die unterschiedlichen Positionen der beteiligten Institutionen - zumindest in den niedersächsischen Büchern - kaum hervor (vgl. Bischewski 2020).

In Hinblick auf die Darstellung aktueller und zukünftiger Krisen, wie dem Ausstieg Großbritanniens aus der EU, ist es zudem wünschenswert, auch die Gründe für die Ablehnung der EU und für Missverständnisse zwischen den europapolitischen Eliten und den EU-Bürgerinnen und -Bürgern bezüglich der Entwicklung der EU zu thematisieren. Denn in der Darstellung der Verfassungsdebatte wurden diese - sofern überhaupt genannt - meist pauschalisiert und nicht erläutert (vgl. Bischewski 2020). In der Vermittlung der EU im Unterricht sollte dementsprechend darauf geachtet werden, dass diese augenscheinliche Reibungslosigkeit - je nach Anforderungsniveau - dekonstruiert bzw. auf gegensätzliche Textabschnitte im Schulbuch (wenn vorhanden) hingewiesen wird.

Trotz der generellen Einigkeit über die Vorteile der europäischen Einigung und der konfliktfreien Darstellung des Integrationsprozesses, die in den Büchern vorherrschen, werden auch Vorurteile über den aktuellen Zustand der EU in den Büchern aufgegriffen. Kritik an der europäischen Bürokratie, "Eurokratie« oder »Technokratie« stehen dabei im Vordergrund und kommen unabhängig von Verlag und Schulform vor. Die als Sinnbild der europäischen "Regelungswut» kritisierte, aber 2009 abge- 
schaffte "Gurkenverordnung" der EU, wird in drei der niedersächsischen Bücher thematisiert. In Mensch und Politik (2007) wird das Beispiel der "Gurkenverordnung" genutzt, um die Vorurteile über scheinbar sinnlose Verordnungen und Richtlinien der EU aufzubrechen. Diese auf einer Doppelseite abgedruckten Dekonstruktionen von bekannten Vorurteilen zur EU sind einmalig in den untersuchten Büchern für Niedersachsen. In Durchblick (2010) findet diese Dekonstruktion dagegen nicht statt, sondern es wird neben einem Foto einer Kiste Gurken auf die Verordnung hingewiesen. Auch im IGL-Buch (2011) wird die Gurkenrichtlinie als Aufhänger verwendet, und zwar mit einer Karikatur, auf der gekrümmte Gurken in Anzügen zu sehen sind. Zwar wird im Autorentext erläutert, dass die besagte Richtlinie nicht mehr existiert, mehr Raum (und daher potentiell mehr Aufmerksamkeit) nimmt jedoch die Karikatur ein.

Auf die europäische Bürokratie gehen insgesamt 18 der 30 niedersächsischen Bücher ein. Dabei wird in vielen Fällen das Vorurteil bedient, dass es sich bei der EU um ein "Bürokratiemonster" handelt, in dem die Regelungswut europäischer Bürokraten herrsche. Karikaturen zeigen Bilder der EU als mächtigem »Bürokrator«, aber auch das Bild überforderter EU-Beamter. Die bürokratischen "Superstrukturen« werden als Grund für die Distanz der EU zu den Bürgerinnen und Bürgern angeführt, die sich mit den Entscheidungen nicht identifizieren können. Einige Bücher greifen das Vorurteil auf, dass die EU lebensferne Entscheidungen träfe, und entkräften es mit Beispielen für EU-Richtlinien und Verordnungen, die den Alltag der Menschen direkt betreffen und so deren Relevanz verdeutlichen. Allerdings wird in keinem der niedersächsischen Bücher darauf eingegangen, dass die Kosten für die EU-Bürokratie tatsächlich viel geringer sind als die der einzelnen Nationalstaaten.

Weniger häufig, aber dennoch in den niedersächsischen Büchern präsent, ist das Vorurteil, dass Deutschland als Nettozahler für die EU herhalten muss, meist untermalt mit der Karikatur eines Esels, der Geldscheine vertilgt. Allerdings wird das Nettozahler-Vorurteil stets mit einem Fragezeichen versehen und im Text durch Verweise auf die notwendige Solidarität zwischen den Mitgliedstaaten (Durchblick 2005: 174) und die Information über die verhältnismäßig höheren Zahlungen anderer Mitgliedstaaten wie etwa Schweden (IGL-Buch 2011: 188) entkräftet. 
Region, Nation, EU - ich? Lebensweltbezug und Schülerorientierung

Der folgende Abschnitt widmet sich den Aspekten Lebensweltbezug und Schülerorientierung. Diese wurden in der Befragung von Tatje (2017) sowohl von den Schülerinnen und Schülern als auch von den Lehrkräften in den Schulbüchern für das Gymnasium und die integrierte Gesamtschule negativ bewertet. Daher soll an dieser Stelle ein Einblick in die Inhalte der Schulbücher gegeben werden, als Grundlage für die Entwicklung möglicher Empfehlungen zur Verbesserung. Dafür wird in den Blick genommen, wie in den niedersächsischen Büchern das Thema "EU im Alltag" präsentiert wird und wie regionale Beispiele genutzt werden. Außerdem wird auf die Darstellungen von Jugendlichen in den EU-Kapiteln eingegangen: Werden ihre Perspektiven und Meinungen zur EU deutlich? Werden EU-Maßnahmen im Bereich Jugend und Bildung vorgestellt? Gibt es Fotos von (weiblichen und männlichen) Jugendlichen? Und letztlich: Wie werden den Lernenden die Möglichkeiten der politischen Partizipation auf europäischer Ebene nahegebracht?

Bei der Behandlung des Themas »Europa im Alltag" wird in den Büchern Team (2010), Politik (2003) sowie Politik-Wirtschaft (2007) von Schöningh das Beispiel des fiktiven »Herrn Kleinschmidt« genutzt, der starke Vorurteile gegenüber der EU hegt und die Behauptung aufstellt, er habe mit der EU nichts zu tun. Im Autorentext wird dagegen erläutert, dass die EU viele bedeutsame Regelungen trifft, die den Alltag einer jeden Bürgerin und eines jeden Bürgers direkt berühren. Ein weiterer Zugang, um die Relevanz der EU im Alltag zu veranschaulichen, ist das Beispiel des Einkaufens im Supermarkt und der Verfügbarkeit von Produkten aus ganz Europa (Trio 2003, Entdecken und Verstehen 2006, Demokratie heute 2009). Diese Thematik wird oftmals verbunden mit einer Aufgabenstellung, die die Schülerinnen und Schüler auffordert, eine eigene Recherche in den Supermärkten vor Ort vorzunehmen. In Büchern für die Hauptschule und Gesamtschule kommt dieser Zugang häufiger vor als in den Büchern für das Gymnasium, was auch mit den jeweiligen curricularen Vorgaben zusammenhängt (vgl. Bischewski 2020). In den Gymnasialbüchern wird hingegen eher die hohe Anzahl an EU-Richtlinien betont, die den Alltag der EUBürgerinnen und -Bürger betreffen. In mehreren Büchern werden die Vorteile der EU aufgegriffen und dabei auf die vier Grundfreiheiten und den 
EU-Binnenmarkt hingewiesen (z. B. Demokratie heute 2009, Politik.21 2012, Politik 2003, Politik-Wirtschaft 2013, Mensch und Politik 2007 und 2012). Eine weitere Möglichkeit, den Bezug der EU zum Alltag zu verdeutlichen, ist die Abbildung von Umfrageergebnissen (meist als Balkendiagramme) zu der Frage »Was bedeutet die EU für Sie?« (z.B. Politik \& Co. 2007 und Anstöße 22010 und 2013). Die niedersächsischen Schulbücher enthalten zur Konkretisierung durchaus regionale Beispiele aus dem Bundesland. Den größten Regionalbezug haben jedoch die Bücher aus Sachsen, in denen dem Thema "Sachsen in der EU« jeweils ein eigener thematischer Abschnitt vorbehalten ist.

Die Darstellung von Jugendlichen und deren Bezug zu Europa wurde ebenfalls qualitativ und quantitativ untersucht. Der Blick in die Inhaltsverzeichnisse der EU-Kapitel (Grundlage: NIS) verrät bereits, dass es zwischen 2003 und 2013 nur wenige Bücher gab, die dem Thema »Jugend und Europa « einen eigenen thematischen Abschnitt widmen, von den aktuelleren Büchern ab 2009 sind es sogar nur noch zwei (Anstöße 22010 und Demokratie heute 2009). In der qualitativen Untersuchung wurden unter dem Ober-Code »Schülerorientierung « unter anderem Meinungen zur EU und Perspektiven von Jugendlichen auf Europa codiert, die in der Mehrzahl der niedersächsischen Schulbücher nachgewiesen werden konnten (23 von 30). Darunter fallen Beiträge fiktiver Art, die in Form von Zitaten oder in einer Sprechblase abgedruckt sind und zum Beispiel durch die Angabe des Alters oder des Hinzufügens eines Fotos als Äußerung eines jungen Menschen identifizierbar sind. Diese kommen besonders häufig in den niedersächsischen Büchern für die Realschule, die Hauptschule und die Gesamtschule vor. Auffällig ist, dass in den Gymnasialbüchern deutlich weniger Textstellen codiert wurden, in denen Meinungen von Jugendlichen präsentiert werden. Inhaltlich handelt es sich bei den Beiträgen hauptsächlich um Erfahrungsberichte oder kurze Schilderungen, die veranschaulichen, welche Erlebnisse die Jugendlichen selber (oder auch ihre Verwandten) mit den vier Grundfreiheiten der EU gemacht haben. In den Büchern für die Hauptschule und Gesamtschule kommen außerdem Berichte zum Thema Berufsausbildung vor, die teils in einem anderen Land der EU absolviert wird. Neben diesen Erfahrungsberichten gibt es aber auch tatsächliche Meinungsäußerungen von Jugendlichen zur EU und deren zukünftiger Entwicklung, wenn auch seltener. Etwas elaborierter fallen Materialien aus, 
die Meinungsumfragen unter Jugendlichen zu ihrer Einstellung zur EU behandeln, wie etwa die Shell Jugendstudie; derartiges kommt aber nur in wenigen Büchern vor. Neben den Meinungen und Perspektiven von Jugendlichen wurden zum Thema "Schülerorientierung" auch Textstellen erfasst, die sich auf EU-Jugendprogramme, EU-Wettbewerbe und EU-Informationsportale sowie Jugendbegegnungen, Austausch und Exkursionen (z.B. nach Brüssel) beziehen. Zwei der vier niedersächsischen Bücher, in denen keiner der Subcodes der Kategorie "Schülerorientierung" vergeben wurde, waren 2015 für das Gymnasium in Niedersachsen zugelassen (Politik-Wirtschaft 2013 und Politik \& Co. 2013). Diese unzureichende Darstellung des Themas "Jugend und Europa« könnte eine Erklärung für die schlechten Bewertungen des Lebensweltbezugs und der Schülerorientierung in der Wirkungsstudie von Tatje sein (siehe Kapitel III).

Auf quantitativer Ebene wurde die thematische Kategorie »Kultur/Bildung/Jugend " (im Sinne des Politikbereiches) erfasst. Zwischen 2003 und 2008 lassen sich in 43,8 \% der Schulbücher quantitativ keine Inhalte zum Thema nachweisen, von 2009 bis 2012/13 sind es $41 \%$, die ohne Begriffe wie etwa "Comenius", "Leonardo", "Weiterbildung", "Jugendaustausch", "Bildungsprogramm« oder »Kulturhauptstadt» auskommen - durchschnittlich werden nur ca. drei Begriffe pro EU-Kapitel genannt. Im Gruppenvergleich der Schulformen zeigen sich dabei keine großen Unterschiede in den Anteilen zum Thema "Kultur/Bildung/Jugend", mit Ausnahme der Schulbücher, die in verschiedenen Schulformen mit Gymnasium zugelassen sind und die anteilsmäßig etwas mehr Schlagworte aufweisen als die Bücher der anderen Schulformen. Bei den Verlagen C.C. Buchner, Klett und Cornelsen finden sich in jeweils knapp der Hälfte der Bücher Schlagworte aus dieser Kategorie, die höchsten Anteile an Büchern mit Schlagworten zum Thema haben Schroedel (77 \%) und Westermann (70 \%). Dagegen gibt es in nur drei der elf untersuchten EU-Kapitel von Schöningh Hinweise auf den Politikbereich »Kultur/Bildung/Jugend « (27\%).

Die Verwendung von Fotos, auf denen Jugendliche abgebildet sind, wird von den Schülerinnen und Schülern keineswegs als "albern" empfunden, sondern eher begrüßt, so ein Ergebnis der Studie von Tatje (2017). Daher kann die Verwendung von entsprechenden Fotos als Mittel zur Verbesserung der Schülerorientierung und des Lebensweltbezuges in den EUKapiteln angesehen werden. Generell kommen (im DEUS) Fotos von Ju- 
gendlichen in einer gemischt-geschlechtlichen Gruppe in den EU-Kapiteln etwas häufiger vor als Bilder, auf denen ausschließlich Mädchen oder Jungen zu sehen sind. Dabei erhöht sich die durchschnittliche Anzahl der abgedruckten Bilder leicht ab 2009 (vgl. Abbildung 6), was damit zu erklären ist, dass nun generell in mehr Ausgaben Bilder von Jugendlichen abgedruckt werden. So gibt es ab 2009 nur noch in $26 \%$ der Bücher keine Fotos von Jugendlichen (in einer gemischt-geschlechtlichen Gruppe), während es vor 2009 noch $46 \%$ waren.

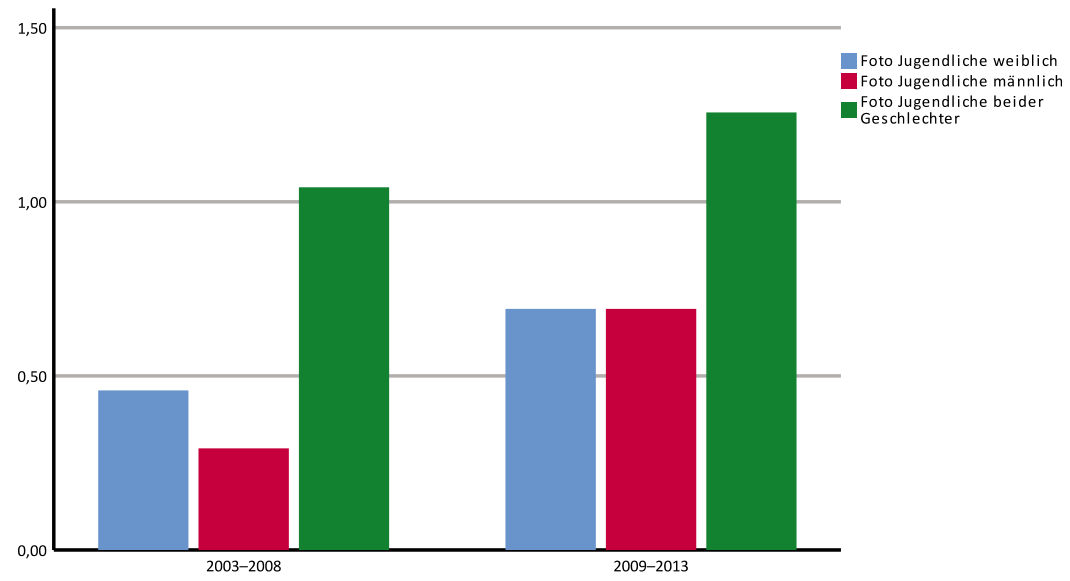

Abbildung 6: Fotos von Jugendlichen nach Geschlecht im Zeitvergleich (2003-2008 versus 2009-2013)

Fasst man die Kategorien zusammen, so kommen bei den größeren Verlagen (Cornelsen, Klett, Schroedel und Schöningh) pro Ausgabe durchschnittlich 2,5 Fotos von Jugendlichen vor. ${ }^{10}$ Bei den Ausgaben der kleinen Verlage (Auer, Oldenbourg und Militzke) fällt auf, dass in ihnen keine Fotos von Jugendlichen zu finden sind. Klett ist der Verlag mit dem größten Anteil an Ausgaben, in denen Fotos von Jugendlichen im EU-Kapitel abgebildet sind, nämlich $91 \%$. Der Gruppenvergleich nach Schulformen zeigt, dass Fotos von Jugendlichen in $52 \%$ der Bücher für das Gymnasium vorkommen, dieser Anteil ist bei den anderen Schulformen deutlich größer.

10 Standardabweichungen: Cornelsen: 1,1; Klett: 1,4; Schroedel: 2,8; Schöningh: 2,9. 
Während in den Realschulbüchern durchschnittlich 3,3 Fotos (SD = 2,9) abgebildet sind, sind es in denen für das Gymnasium nur 1,6 ( $\mathrm{SD}=2,2)$.

Abschließend soll diskutiert werden, wie die Möglichkeiten der politischen Partizipation auf europäischer Ebene in den Schulbuchtexten dargestellt werden. Die geringe Beteiligung der Bevölkerung an den Wahlen zum Europäischen Parlament wird seit Jahren kritisiert. Dabei stellt die Direktwahl des Europäischen Parlaments die zentrale Möglichkeit der politischen Teilhabe für die EU-Bürgerinnen und -Bürger dar und ist notwendiges Mittel der europäischen Input-Legitimierung. Voraussetzung für die Ausbildung der von der politischen Bildung gewünschten Handlungsfähigkeit ist zunächst das Wissen um die bestehenden Partizipationsmöglichkeiten (vgl. Engartner et al. 2015). Daher ist es überraschend, wie gering der Anteil in den EU-Kapiteln zu den Themen "Europawahl« und »Bürgerpartizipation« ausfällt. Die quantitative Inhaltsanalyse des DEUS zeigt deutlich, dass das Thema »Europawahl« in den Kapiteln zur EU nur gering vertreten ist und dass sich sein Anteil im Zeitverlauf zudem etwas verringert. Unter den aktuellen Schulbüchern gibt es nur wenige Ausgaben, die sich dieser Thematik stärker widmen. Die Ergebnisse der quantitativen Analyse bestätigen die Beobachtungen der qualitativen Analyse, dass dem Thema "Europawahl« in den EU-Kapiteln nur ein geringer Raum beigemessen und teilweise sogar nur indirekt auf die Wahl des Europäischen Parlaments verwiesen wird (vgl. Bischewski 2020). Positiv hervorzuheben ist jedoch, dass der Anteil der Bücher, die die Europawahl gar nicht thematisieren, von 20,8 \% (zehn Fälle) zwischen 2003 und 2008 auf 5,1 \% (zwei Fälle) zwischen 2009 und 2012/13 gesunken ist. Wie häufig bzw. ausführlich die Europawahl thematisiert wird, ist je nach Verlag unterschiedlich. Die Verlage mit den höchsten Mittelwerten in der Kategorie "Anteil Europawahl" sind Militzke $(M=0,10)$, Bildungsverlag Eins $(M=0,10$; nur ein Buch), Cornelsen $(M=0,08)$. Die Werte der anderen, größeren Verlage liegen deutlich darunter. Auch beim Thema »Bürgerpartizipation« (erfasst mit Schlagworten wie »Demonstration «, »Referendum «, »Bürgerinitiative« etc.) sind es insbesondere die kleineren Verlage und Cornelsen, die höhere Mittelwerte aufweisen. Allerdings muss insbesondere bei Verlagen mit vielen Ausgaben (wie Schroedel) die hohe Spannweite beachtet werden, die bedeutet, dass es durchaus Ausgaben mit Schwerpunkten zum Thema »Europawahl» gibt, diese jedoch nicht die Masse der Bücher ausmachen. 
Was die Schulformen betrifft, so sind die Anteile zur "Europawahl « in den Büchern für die Realschule $(M=0,05)$ und das Gymnasium $(M=0,04)$ höher als in den anderen Schulformen. Zu dem Thema "Bürgerpartizipation" findet sich in den Büchern für das Gymnasium $(M=0,02)$ jedoch weniger als in den Büchern für die Realschule $(M=0,04)$, die Hauptschule $(M=0,04)$, oder verschiedene Schulformen ohne Gymnasium $(M=0,04)$.

\section{II.3 Zusammenfassung}

Die Befunde der produktorientierten Teilstudie zeichnen ein sehr heterogenes Bild der Darstellung der EU in deutschen Politikschulbüchern zwischen 2003 und 2012/13, wobei sich die Inhalte in Schulbüchern insgesamt bereits innerhalb einer Dekade deutlich verändert haben. Ereignisse, die einen besonderen Einfluss auf eine Veränderung der Gewichtung (Häufigkeit) und der inhaltlichen Darstellung bestimmter Themen hatten, waren zum einen die Ratifizierung des Vertrags von Lissabon und zum anderen die Einführung von neuen Kerncurricula. Dabei zeigen die quantitativen und qualitativen Analysen, dass sowohl die Häufigkeit als auch die inhaltliche Aufbereitung von Themen nach Verlagen, Bundesländern und Schulformen variiert. Doch auch innerhalb der Verlage sind die Unterschiede in der Qualität der Darstellung und der Quantität der Gegenstände hoch. Insgesamt verdeutlicht die Untersuchung, dass in vielen Schulbüchern eine "Reibungslosigkeit" des europäischen Integrationsprozesses suggeriert wird und ein generelles »Nicht-in-Frage-stellen« der Europäischen Union vorherrscht. Auch der Charakter des europäischen Mehrebenensystems wird nicht hinreichend deutlich und Fachbegriffe werden zu wenig genutzt bzw. unzureichend erläutert. Schließlich werden die auf europäischer Ebene bestehenden Partizipationsmöglichkeiten, inklusive der Wahlen zum Europäischen Parlament, überraschend selten näher behandelt. 


\section{Literatur}

Baros, Wassilios und Eva Wilke. »Die Europäische Union im Schulbuch aus der Sicht von Schülerinnen und Schülern im internationalen Vergleich - eine Latent-ClassAnalyse«, in: Methodologie und Methoden der Schulbuch- und Lehrmittelforschung, Eva Matthes und Sylvia Schütze (Hg.), Bad Heilbrunn: Klinkhardt, 2015.

Bischewski, Marret. Von Laeken bis Lissabon: Der Reformprozess der Europäischen Union in deutschen Politikschulbüchern. Eine computergestützte Inhaltsanalyse, Dissertation, Göttingen: Georg-August-Universität Göttingen, 2020.

Challand, Benoît. »European Identity and External Others in History Textbooks (1950-2005)«, in: Journal of Educational Media, Memory, and Society 1 (2), 2009, 60-96, doi:10.3167/jemms.2009.010204.

Detjen, Joachim. ")Europäische Unübersichtlichkeiten`. Wie soll die politische Bildung mit der Kompliziertheit und Intransparenz der Europäischen Union umgehen?«, in: Europa verstehen lernen. Eine Aufgabe des Politikunterrichts, Georg Weißeno (Hg.), Bonn: Bundeszentrale für Politische Bildung, 2004, 126143.

Eis, Andreas. Europäische Bürgerschaftsbildung. Die Neukonstruktion der Bürgerrolle im europäischen Mehrebenensystem, Schwalbach/Ts.: Wochenschau Verlag, 2010.

Engartner, Tim, Markus B. Siewert, Maria Theresa Meßner und Christiane Borchert. "Politische Partizipation sspielend fördern? Charakteristika von Planspielen als didaktisch-methodische Arrangements handlungsorientierten Lernens«, in: Zeitschrift für Politikwissenschaft 25 (2), 2015, 189-217, doi:10.5771/ 1430-6387-2015-2-189.

Europäische Union. »Konsolidierte Fassung des Vertrags über die Europäische Union und des Vertrags über die Arbeitsweise der Europäischen Union«, in: Amtsblatt der Europäischen Union 53 (C 83), 2010, http://eur-lex.europa.eu/legalcontent/DE/TXT/PDF/?uri=OJ:C:2010:083:FULL\&from=DE, zuletzt geprüft am 05. September 2020.

Höhne, Thomas. Schulbuchwissen. Umrisse einer Wissens- und Medientheorie des Schulbuches, Frankfurt/M.: Fachbereich Erziehungswissenschaft der Johann-Wolfgang-Goethe-Universität, 2003.

Karayianni, Eleni. "The place of Europe in English history textbooks«, in: Representations of otherness. The Eleventh International Conference on Research on Textbooks and Educational Media, Natalija Mazeikiene, Mike Horsley und Susanne V. Knudsen (Hg.), Noosa: Central Queensland University, 2011, 59-68.

Kuckartz, Udo. Qualitative Inhaltsanalyse. Methoden, Praxis, Computerunterstützung, 2. Aufl., Weinheim: Beltz Juventa, 2014. 
Lässig, Simone. "Textbooks and Beyond: Educational Media in Context(s)«, in: Journal of Educational Media, Memory, and Society 1 (1), 2009, 1-20, doi:10.3167/ jemms.2009.010101.

Lamnek, Siegfried. "Qualitative und quantitative Inhaltsanalyse: Forschungsmethoden im Kontext von Schulbuchanalysen zum Geschichtsunterricht", in: Die religiöse Dimension im Geschichtsunterricht an Europas Schulen. Ein interdisziplinäres Forschungsprojekt, Waltraud Schreiber (Hg.), Neuried: ars una, 2000, 319-348.

Mahamud, Kira. »Contexts, Texts and Representativeness. A Methodological Approach to School Textbook Research", in: Methodologie und Methoden der Schulbuch- und Lehrmittelforschung, Eva Matthes und Sylvia Schütze (Hg.), Bad Heilbrunn: Klinkhardt, 2015.

Mayring, Philipp. Einführung in die qualitative Sozialforschung. Eine Anleitung zu qualitativem Denken, 5. Aufl., Weinheim: Beltz, 2002.

Mellies, Dirk, Pawel Migdalski, Christoph Kühberger u. a. Empfehlungen zum Umgang mit Geschichte anhand von Schulbuchkapiteln zur Geschichte der Europäischen Union, 2007, http://www.demokratiezentrum.org/fileadmin/media/pdf/ku ehberger_eu.pdf, zuletzt geprüft am 05. September 2020.

Natterer, Alexandra. Europa im Schulbuch. Die Darstellung der europäischen Einigung in baden-württembergischen Schulbüchern für Geschichte und Gemeinschaftskunde der Sekundarstufe I, Grevenbroich: Omnia, 2001.

Oberle, Monika und Kerstin Pohl. »Politik in der Lehrerinnen- und Lehrerbildung Professionalisierung für ein vielgestaltiges Unterrichtsfach «, in: Handbuch Lehrerinnen- und Lehrerbildung, Colin Cramer, Johannes König, Martin Rothland und Sigrid Blömeke (Hg.), Bad Heilbrunn/Stuttgart: Klinkhardt/UTB.

Pingel, Falk. Macht Europa Schule? Die Darstellung Europas in Schulbüchern der Europäischen Gemeinschaft, Frankfurt/M.: M. Diesterweg, 1995.

Ders. "How to approach Europe? The European Dimension in History Textbooks«, in: Grenzgänger/Transcending Boundaries, ders., (Hg.), Göttingen: V\&R unipress, 2009, 358-402.

Pingel, Falk und Michail Boitsev. The European home. Representations of 20th century Europe in history textbooks, Straßburg: Council of Europe Pub., 2000.

Pohl, Kerstin. "Schulischer Fachunterricht«, in: Handbuch politische Bildung, Bd. 69, 4. Aufl., Wolfgang Sander (Hg.), Schwalbach/Ts.: Wochenschau Verlag, 2014, 186-193.

Schmit, Stefan. »Heuristischer Entwurf eines basalen Untersuchungsdesigns für die Analyse von Schulbüchern und Anwendung bei der Untersuchung der Eignung von Physikschulbüchern als Lernmaterialien«, in: Methodologie und Methoden der Schulbuch- und Lehrmittelforschung, Eva Matthes und Sylvia Schütze (Hg.), Bad Heilbrunn: Klinkhardt, 2015, 50-62. 
Slopinski, Andreas und Torsten J. Selck. "Wie lassen sich Wertaussagen in Schulbüchern aufspüren? Ein politikwissenschaftlicher Vorschlag zur quantitativen Schulbuchanalyse am Beispiel des Themenkomplexes der europäischen Integration «, in: Journal of Educational Media, Memory, and Society, 6 (1), 2014, 124-141, https://doi.org/10.3167/jemms.2014.060107.

Tatje, Christian. Die Rolle des Schulbuchs bei der Vermittlung der Europäischen Union. Nutzung und Wirkung im politischen Fachunterricht, Wiesbaden: Springer VS, 2017.

Weißeno, Georg. "Konturen einer europazentrierten Politikdidaktik - Europäische Zusammenhänge verstehen lernen«, in: Europa verstehen lernen. Eine Aufgabe des Politikunterrichts, ders. (Hg.), Bonn: Bundeszentrale für Politische Bildung, 2004, 108-125.

Ders. »Fachsprache in Schulbüchern für Politik/Sozialkunde: eine empirische Studie", in: Demokratischer Verfassungsstaat und politische Bildung, Peter Massing und Georg Weißeno (Hg.), Schwalbach/Ts.: Wochenschau Verlag, 2013, 151-170.

Wettstein, Martin. "Best of both worlds. Die halbautomatische Inhaltsanalyse», in: Automatisierung in der Inhaltsanalyse, Bd. 11, Katharina Sommer, Martin Wettstein, Werner Wirth und Jörg Matthes (Hg.), Köln: von Halem, 2014, 16-39. 
Open-Access-Publikation im Sinne der CC-Lizenz BY 4.0

(C) 2021, Vandenhoeck \& Ruprecht $\mathrm{GmbH} \&$ Co. KG, Göttingen ISBN Print: 9783847112655 - ISBN E-Lib: 9783737012652 


\section{Die prozess- und wirkungsorientierte Teilstudie: Nutzung und Bewertung der EU-Kapitel durch Lehrkräfte sowie Schülerinnen und Schüler}

Wie eingangs dargestellt, mangelt es an systematischen Analysen der tatsächlichen Verwendung des Schulbuchs bei der Planung und Gestaltung des Politikunterrichts im Allgemeinen und des EU-Unterricht im Speziellen. Auch fehlen bislang Untersuchungen der Qualität der Politik-Vermittlung in Schulbüchern bzw. in den EU-Kapiteln aus Sicht von Lehrkräften sowie Schülerinnen und Schülern. Diesen Desiderata begegnet die im Folgenden vorgestellte zweite Teilstudie des Forschungsprojekts. Untersucht wurde die tatsächliche Nutzung (Häufigkeit und Funktionen) des Politikschulbuchs und seiner Bestandteile durch Lehrkräfte und Lernende vor, während und nach dem Unterricht. Weinbrenner (1995) differenziert drei verschiedene Ansätze der Schulbuchforschung: die prozessorientierte Schulbuchforschung, die sich mit dem Lebenszyklus des Schulbuches befasst (Entwicklung, Zulassung, Vermarktung sowie die Art und Weise der Verwendung); die produktorientierte Schulbuchforschung, welche in der Regel inhaltsanalytisch vorgeht, sowie die wirkungsorientierte Schulbuchforschung, die als Schul- bzw. Unterrichtsforschung oder auch als Medien-, Kommunikations- und Rezeptionsforschung verstanden werden kann. Hier wird unterschieden zwischen der Wirkung auf Lernende (z. B. ob die Darstellung von Themen für die Verständnisebene der Schülerinnen und Schüler angemessen ist) und der Wirkung auf Lehrkräfte (z. B. ob das Buch der fachlichen bzw. didaktisch-methodischen Intention der Lehrperson entspricht). Die vorliegende Teilstudie ist damit der prozessorientierten und der wirkungsorientierten Schulbuchforschung zuzuordnen. 


\section{III.1 Ziele und Fragestellungen}

Ziel dieser Teilstudie ist es, belastbare Erkenntnisse hinsichtlich der Nutzung und Beurteilung von Schulbüchern des politischen Fachunterrichts und deren EU-Kapitel zu erlangen. Erstmals soll damit ein systematischer Einblick in das tatsächliche Nutzungsverhalten von Schülerinnen und Schülern sowie Lehrkräften in Bezug auf das Politikschulbuch und dessen Inhalte ermöglicht werden. Dabei wird insbesondere den Fragen nachgegangen, wie intensiv und wofür die Bücher seitens der Lernenden und Lehrenden verwendet werden. Von Relevanz ist außerdem, welche Bücher eingesetzt werden. Des Weiteren soll beleuchtet werden, wie die Politikschulbücher von den beiden untersuchten Gruppen beurteilt werden. Schließlich wird überprüft, inwiefern verschiedene potenzielle Einflussfaktoren wie sozio-demografische Hintergrundvariablen (z.B. Geschlecht, Migrationshintergrund) der Lernenden und Lehrenden sowie die lehr-lerntheoretischen Überzeugungen der Lehrkräfte Einfluss auf die Nutzung und Beurteilung des Politikschulbuchs haben.

\section{III.2 Design der Studie}

Die Hauptstudie bestand aus einer teilstandardisierten schriftlichen Befragung von Schülerinnen und Schülern $(\mathrm{n}=1076)$ und (unter anderem deren) Politiklehrkräften $(\mathrm{n}=123)$ der Sekundarstufe I an Gymnasien und Gesamtschulen, die von geschulten Versuchsleiterinnen und Versuchsleitern durchgeführt wurde. Zur Erstellung der Lehrerfragebögen erfolgte zunächst eine qualitative Vorstudie in Form von leitfadengestützten Interviews (Herbst 2014, $\mathrm{n}=16$ ). Der Schülerfragebogen wurde in einer Vorstudie (Frühjahr 2014, $\mathrm{n}=282$ ) getestet, die durch zahlreiche offene Fragen die Generierung weiterer Hypothesen und Items ermöglichte. Die Gesamtstudie folgt damit einem Mixed-Methods-Ansatz im sequenziellen Design (vgl. Abbildung 7) und trianguliert Schüler- und Lehrerangaben zur Nutzung des Politikschulbuchs (vgl. Flick 2011).

Die Datenerhebung der Hauptstudie erfolgte 2015. In der Schülerstudie wurden $\mathrm{n}=1076$ Schülerinnen und Schüler aus 52 Klassen an 18 niedersächsischen Sekundarschulen befragt: 51,9 \% weiblich, 48,1 \% männlich; 


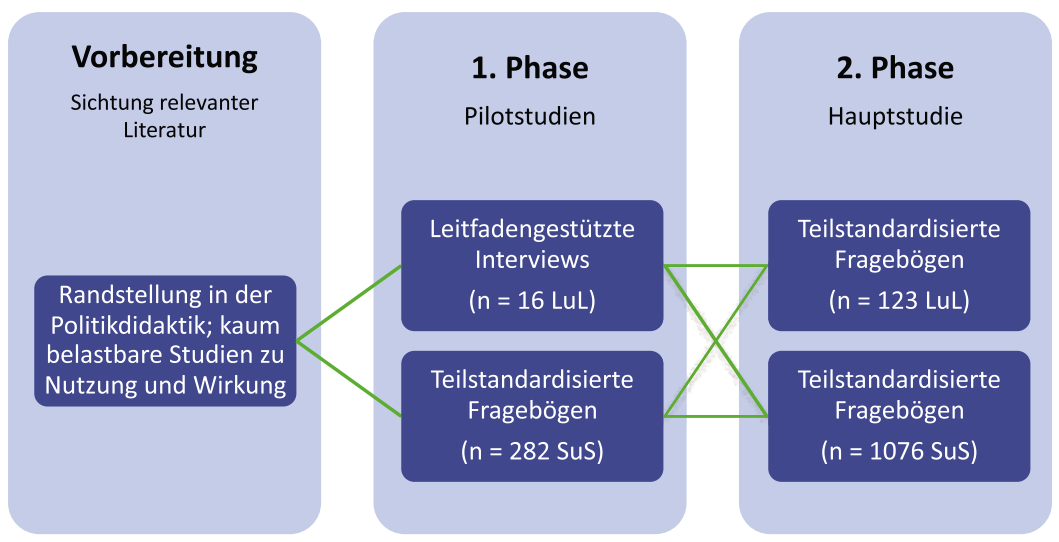

Abbildung 7: Design der Studie

Durchschnittsalter 16,08 Jahre; 21,4 \% mit Migrationshintergrund (Kind oder mind. ein Elternteil im Ausland geboren); 84,6 \% aus der 10. Klasse eines Gymnasiums, $15,4 \%$ aus der 11. Klasse einer Gesamtschule. ${ }^{11}$ In allen Klassen wurde die EU zuvor im politischen Fachunterricht behandelt. Die Nutzung eines Schulbuchs bzw. der Umfang einer solchen Nutzung war kein Auswahlkriterium. Jede Schülerin und jeder Schüler hatte während der Befragung (ca. 40-50 Min.) das an der Schule verwendete Politikbuch vorliegen. So konnten Detailfragen zielführender beantwortet werden, selbst bei geringer Nutzung des Buchs im Unterricht.

$\mathrm{Zu}$ jeder befragten Schulklasse liegt auch ein Lehrerfragebogen vor. Insgesamt besteht das Sample der Lehrerstudie aus $\mathrm{n}=123$ Politiklehrkräften von 42 Schulen in Niedersachsen (Schulform: 84,4\% Gymnasium, 9,0 \% Kooperierende Gesamtschule, 2,5 \% Integrierende Gesamtschule; Geschlecht: 57,4 \% männlich, 39,3\% weiblich, 3,3\% k.A.; Altersdurchschnitt: ca. 42 Jahre; Studienfächer: 85,2 \% Politik(-wissenschaft) bzw. Politik-Wirtschaft, 9,0 \% Sozialwissenschaften, 5,8 \% keine fachliche Bezugsdisziplin; Ausbildungsstatus: 13,1 \% im Referendariat, ansonsten

11 Die Zielgruppe ergibt sich daraus, dass das Gesamtprojekt EU-bezogene Fragestellungen fokussiert und die niedersächsischen Kerncurricula zum Erhebungszeitpunkt die Behandlung der EU an Gymnasien (G8) und Gesamtschulen (G9) in den genannten Jahrgangstufen vorsahen. 
praktizierende Lehrkräfte; durchschnittliche Berufserfahrung: 6-10 Jahre). Neben den genannten sozio-demografischen Hintergrundvariablen wurden mit insgesamt neun 4-stufig Likert-skalierten Items (nach Oberle, Weschenfelder und Weißeno 2014) auch lehr-lern-theoretische Überzeugungen (beliefs) der Lehrkräfte erhoben. Diese gelten als Facette der Professionskompetenz von Lehrpersonen, der eine handlungsleitende Funktion attestiert wird (vgl. Oberle, Weschenfelder und Weißeno 2014; Baumert und Kunter 2006). Sie können sich eher an transmissiven (Lernen als Weitergabe von Wissen durch Übung und verstärkendes Feedback) oder eher an konstruktivistischen Lerntheorien (Lernen als aktiver und weitgehend selbstgesteuerter Prozess; vgl. Dubberke et al. 2008 sowie Hartinger, Kleickmann und Hawelka 2006) orientieren, wobei sich die beiden Ausprägungen nicht unbedingt ausschließen. Das vorliegende Modell besteht aus den beiden mit $\mathrm{r}=-.32^{\star * \star}$ negativ korrelierten Faktoren transmissiv (fünf Items, $\alpha=.71$ ) und konstruktiv (vier Items, $\alpha=.70$ ) und weist einen guten Modellfit auf $\left(\mathrm{x}^{2}=32.30(26) \mathrm{ns}, \mathrm{CFI} / \mathrm{TLI}=.98 / .97, \mathrm{RMSEA}=.05\right.$, WRMR $\left.=.64\right)$.

Das Schulbuchnutzungsverhalten sowie die Beurteilungen der Schulbücher von Lernenden und Lehrerkräften wurde mit 4-stufigen Likert-skalierten Items erfragt (Antwortoptionen zur Nutzung: sehr häufig, häufig, selten, nie; Antwortoptionen zur Beurteilung z. B.: sehr gut, eher gut, eher schlecht, sehr schlecht). Die Items wurden eigens für die vorliegende Studie entwickelt (unter anderem mittels der beiden Vorstudien, siehe oben), da eine umfassende Sichtung der Literatur hinsichtlich der Erhebungsinstrumente wenig ergiebig war. Lediglich von Borries (2005) bot in seiner Studie zum Geschichtsschulbuch zumindest eine grobe erste Orientierung für die Formulierung von politikschulbuchbezogenen Items. Mess- und Regressionsmodelle wurden latent in Mplus 7.4 modelliert, wobei Likert-skalierte Items als kategoriale Variablen behandelt wurden und die hierarchische Struktur der Daten berücksichtigt wurde.

\section{III.3 Ergebnisse zur Nutzung}

Bezüglich der Frage, welche Politikschulbücher an niedersächsischen Gymnasien und Gesamtschulen eingesetzt werden, zeigten die Schüler- und die Lehrerstudie sehr ähnliche Ergebnisse. Der Vergleich ist insofern relevant, 
als die Lehrerstichprobe deutlich mehr niedersächsische Schulen repräsentiert (42 Schulen) als die Schülerstichprobe (18 Schulen) - aus den Angaben der Lehrkräfte aber dennoch eine nahezu gleiche Verteilung der Schulbuchverlage abgeleitet werden konnte wie aus den Schülerdaten. Die Abbildung 8 basiert deshalb auf den Angaben der 123 Lehrpersonen aus 42 Schulen, verteilt über ganz Niedersachsen. Zu erkennen ist eine klare Dominanz von C.C. Buchner mit 57,1 \% (Politik \& Co.), gefolgt von Schroedel mit 25,1\% (Mensch \& Politik). Klett (Anstöße 2), Westermann (Kompetenz Politik) und Schöningh (Politik-Wirtschaft) sind jeweils nur an ca. $6 \%$ der befragten Schulen zu finden.

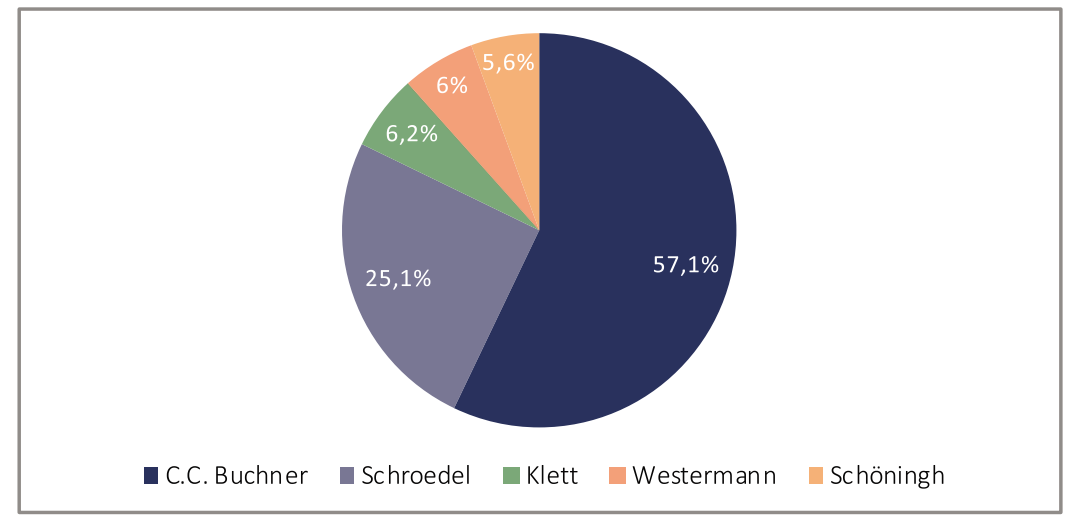

Abbildung 8: Im politischen Fachunterricht niedersächsischer Gymnasien (10. Klasse) und Gesamtschulen (11. Klasse) genutzte Schulbücher nach Verlagen in Prozent (Lehrkräfte, $\mathrm{n}=123$ )

Aufschluss über die tatsächliche Nutzung des Schulbuchs im politischen Fachunterricht und im Unterricht zur EU geben die Antworten der Lernenden und Lehrkräfte auf die Frage, wie häufig sie das Schulbuch im Politikunterricht generell benutzen. Die Angaben erfolgen in Prozent, wobei zunächst nur die Ergebnisse der Lehrkräfte dargestellt sind (vgl. Abbildung 9). Nach eigener Einschätzung nutzen ca. $27 \%$ der Lehrkräfte das Schulbuch im politischen Fachunterricht generell in jeder oder fast jeder Stunde und ca. $44 \%$ in etwa der Hälfte der Stunden. Unter Hinzunahme der dritten Antwortoption »in weniger als der Hälfte der Stunden« (aber öfter als 1-2 Mal pro Schuljahr) wird deutlich, dass mehr als $90 \%$ der Lehrkräfte 
das Politikschulbuch regelmäßig im politischen Fachunterricht einsetzen. Nur eine von 123 Lehrkräften gibt an, gänzlich auf das Schulbuch zu verzichten. Werden die Ergebnisse zum Politikunterricht insgesamt mit der Nutzung des Schulbuchs speziell im EU-Unterricht kontrastiert, zeigt sich, dass erheblich mehr Lehrkräfte es im Unterricht zur EU in jeder oder fast jeder Stunde einsetzen (ca. 38 \%). Somit weist zumindest ein Teil der befragten Personen offensichtlich einen höheren Bedarf am Schulbuch als Leitmedium im Unterricht zur EU auf als im Politikunterricht generell. Insgesamt ist der Anteil der Lehrpersonen, die das Politikschulbuch regelmäßig im EU-Unterricht einsetzen - verglichen mit den Angaben zur generellen Nutzung - mit ca. 84 \% jedoch etwas geringer, da es wiederum einen höheren Anteil an Lehrkräften gibt, die das Politikschulbuch während der EU-Einheit nur 1-2 Mal (ca. 14 \%) oder sogar nie (ca. 3 \%) nutzen.

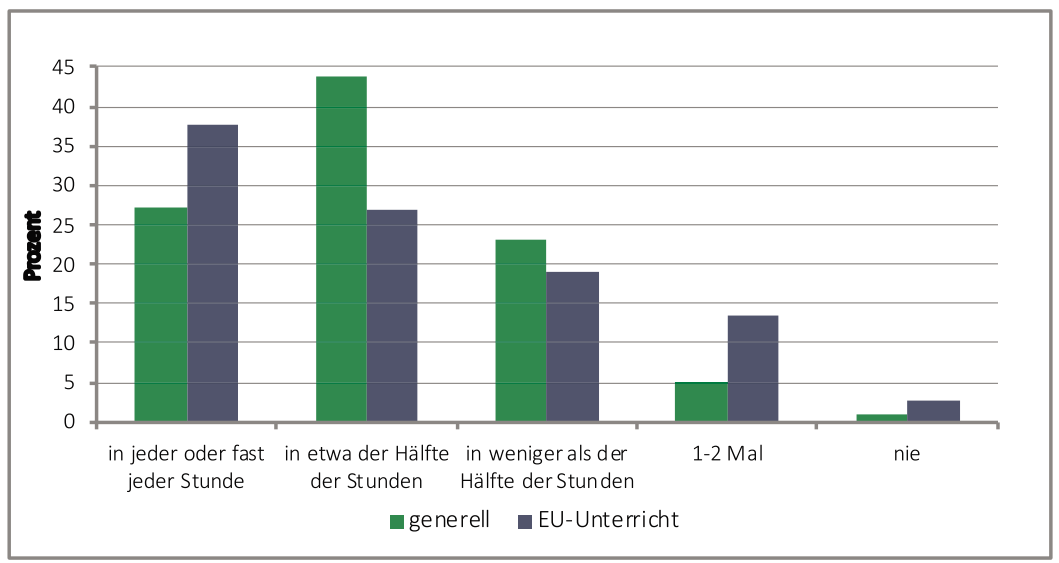

Abbildung 9: Nutzung des Politikschulbuchs generell und im EU-Unterricht (Lehrkräfte, $\mathrm{n}=123)$

Latente multiple Regressionen ergeben, dass Politiklehrerinnen und -lehrer mit eher transmissiven lehr-lerntheoretischen Überzeugungen das Schulbuch häufiger als "Leitmedium« nutzen (Rauch und Tomaschewski 1986), also für die Planung von Unterrichtseinheiten und/oder einzelnen Stunden, während Lehrkräfte mit eher konstruktivistischen bzw. konstruktionsorientierten Überzeugungen das Politikschulbuch tendenziell weniger einsetzen (vgl. auch Oberle und Tatje 2017). 
Die Angaben der befragten Schülerinnen und Schüler zur Nutzung des Politikschulbuchs (vgl. Abbildung 10) zeigen interessanterweise eine geringere Intensität der Nutzung. So geben nur 18,3\% der Lernenden an, das Buch in jeder oder fast jeder Stunde und ca. $30 \%$ es in etwa der Hälfte der Stunden zu benutzen. Unter Hinzunahme der dritten Option ergibt sich somit ein Anteil von $80 \%$, die es nach eigenen Angaben regelmäßig (also öfter als 1-2 Mal) im allgemeinen Politikunterricht nutzen. Im Vergleich zu den Lehrerangaben (ca. $93 \%$ ) fallen die Angaben der Schülerinnen und Schüler also etwas geringer aus. Keine der 52 befragten Klassen arbeitet jedoch ohne das Politikschulbuch. Da die befragten Klassen und Lehrpersonen nicht nach ihrem Schulbuch-Nutzungsverhalten ausgewählt wurden, ist davon auszugehen, dass ein vollständiger Verzicht auf das Lehrwerk im Politikunterricht an niedersächsischen Gymnasien und Gesamtschulen nur äußerst selten vorkommt.

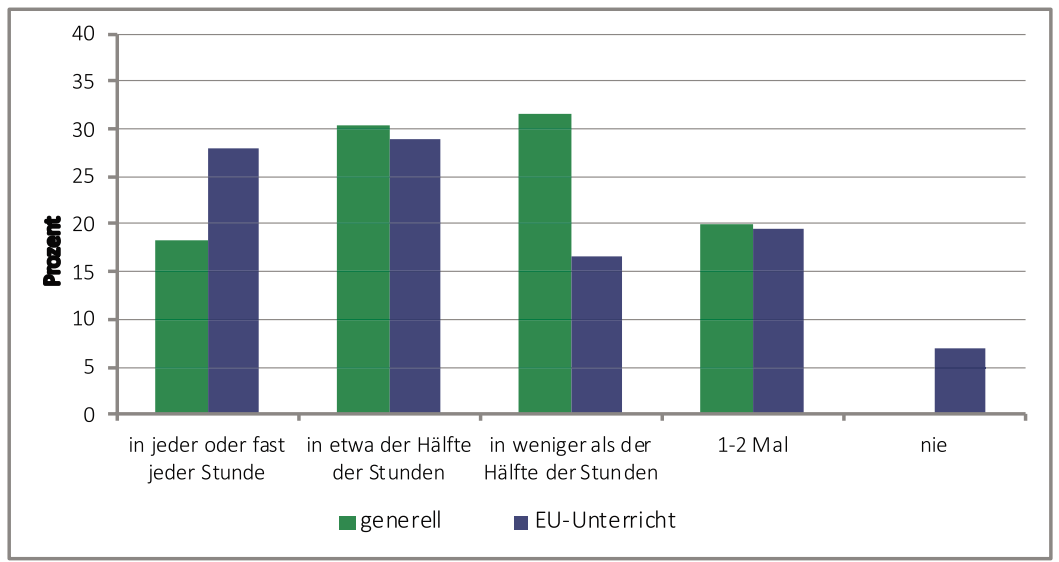

Abbildung 10: Nutzung des Politikschulbuchs generell und im EU-Unterricht (Schülerinnen und Schüler, $\mathrm{n}=1076$ )

Die bei den Lehrkräften bereits ersichtliche Tendenz einer häufigeren Nutzung des Lehrwerks in jeder oder fast jeder Unterrichtsstunde im Kontext des EU-Unterrichts (vgl. Abbildung 9) wird durch die Schülerinnen und Schüler bestätigt (27,9\%). Die daraus resultierende geringere Anzahl derer, die angeben, das Schulbuch in etwa der Hälfte der EU-Stunden zu nutzen $(29,1 \%)$, und der deutliche Abfall in der dritten Antwortkategorie bestäti- 
gen ebenfalls die oben genannten Antworten der Lehrkräfte. Im Gegensatz zum generellen Unterricht gibt es drei Klassen, die das Schulbuch nie im Unterricht zur EU verwendeten.

Grundsätzlich scheinen die Schülerinnen und Schüler den Einsatz des Schulbuchs als weniger häufig zu empfinden als die Lehrkräfte, was unter anderem darauf zurückgeführt werden könnte, dass die Lehrkräfte trotz der Frage nach der Nutzung im Unterricht auch die Vorbereitung bzw. die Verwendung des Schulbuchs als Kopiervorlage bei ihrer Antwort miteinbezogen und die Lernenden nur die für sie im Unterricht wahrnehmbare Nutzungszeit angaben.

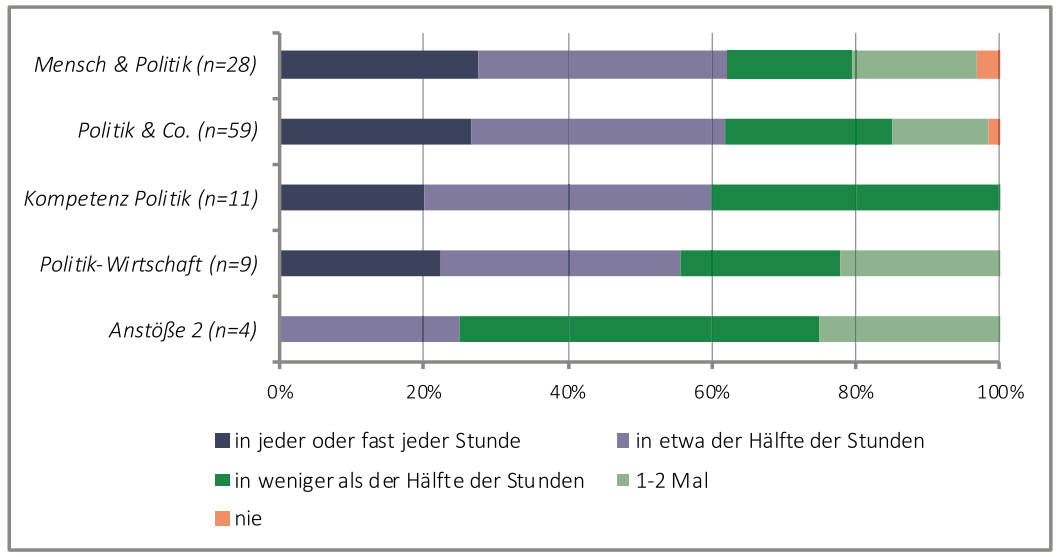

Abbildung 11: Häufigkeit der Nutzung je Politikschulbuch im EU-Unterricht (Lehrkräfte, $\mathrm{n}=123$ )

In Abbildung 11 sind die oben genannten Antwortoptionen zur Nutzungshäufigkeit je Politikschulbuch aufgeführt. Dies soll Aufschluss darüber geben, ob die Nutzung des Schulbuchs im Unterricht zur EU auch davon abhängt, welches Lehrwerk den Unterrichtenden zur Verfügung steht. Die Angaben erfolgen in Prozent und sind aus Perspektive der Lehrkräfte nur für die fünf in dieser Stichprobe am häufigsten vorkommenden Politikschulbücher dargestellt (z. B. wurde Votum nur von einer Person genutzt). Mensch \& Politik sowie Politik \& Co. führen dabei unter Kombination der ersten beiden Antwortoptionen (»in jeder oder fast jeder Stunde « und »in etwa der Hälfte der Stunden«) die Rangliste an. Trotz dieser 
hohen Werte weisen beide Werke jedoch auch als einzige Bücher Werte in der Kategorie "nie» auf. Hier ist anzumerken, dass die beiden Lehrwerke von über der Hälfte der Stichprobe eingesetzt wurde $(n=87)$ und daher die Wahrscheinlichkeit größer ist, dass sich innerhalb dieser Gruppe auch Lehrkräfte befinden, die das Buch sehr selten bzw. gar nicht nutzen. Kompetenz Politik wird seltener in jeder oder fast jeder Stunde eingesetzt, aber unter Hinzunahme der zweiten Antwortoption sind die Werte kaum geringer. Ähnlich verhält es sich mit Politik-Wirtschaft. Das Buch Anstöße 2 wird als einziges Lehrwerk von keiner Person in jeder oder fast jeder Stunde genutzt. Aufgrund der divergierenden Gruppengröße (von $n=4$ bis $n=59$ ) ist diese Darstellung eher als Tendenz zu interpretieren, die bestätigt, dass Politik \& Co. und Mensch \& Politik die höchsten Werte in der häufigsten Nutzungsoption haben.

Zusammenfassend kann zur Nutzung des Politikschulbuchs festgestellt werden, dass das Schulbuch nach wie vor als favorisiertes Unterrichtsmaterial für den Politikunterricht gilt. Ergänzt werden diese Angaben nun noch durch die aus Lehrerperspektive empfundene Wichtigkeit dieses Lehrwerks (vgl. Abbildung 12). So hat das Schulbuch für die Lehrkräfte insbesondere im Kontext der Vorbereitung des politischen Fachunterrichts eine hohe Bedeutung: 26,2 \% empfinden das Buch dabei als sehr wichtig, 43,4 \% als eher wichtig, $28,7 \%$ als eher unwichtig und nur 1,6\% als sehr unwichtig. Während des Unterrichts messen die Politiklehrerinnen und -lehrer dem Schulbuch eine etwas geringere, aber noch immer hohe Bedeutung bei: 20,5 \% empfinden es dabei als sehr wichtig, 47,5\% als eher wichtig, 30,3\% als eher unwichtig und wiederum $1,6 \%$ als sehr unwichtig.

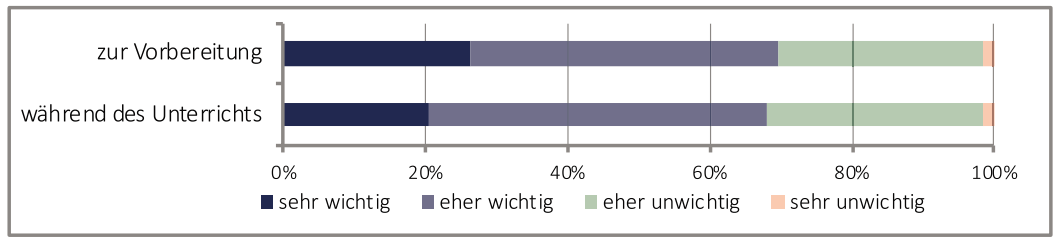

Abbildung 12: Wichtigkeit des Politikschulbuchs (Lehrkräfte, $\mathrm{n}=123$ )

Bei der etwas ausdifferenzierteren Frage nach den Nutzungsarten (vgl. Abbildung 13) lässt sich erkennen, dass mehr als drei Viertel der Lehrkräfte das Schulbuch sehr oder eher häufig für die Planung sowohl der einzelnen 
EU-Stunde als auch der gesamten Unterrichtseinheit nutzen. Etwa die Hälfte nutzt es regelmäßig auch ergänzend und etwa ein Viertel regelmäßig für die Auffrischung der eigenen Kenntnisse.

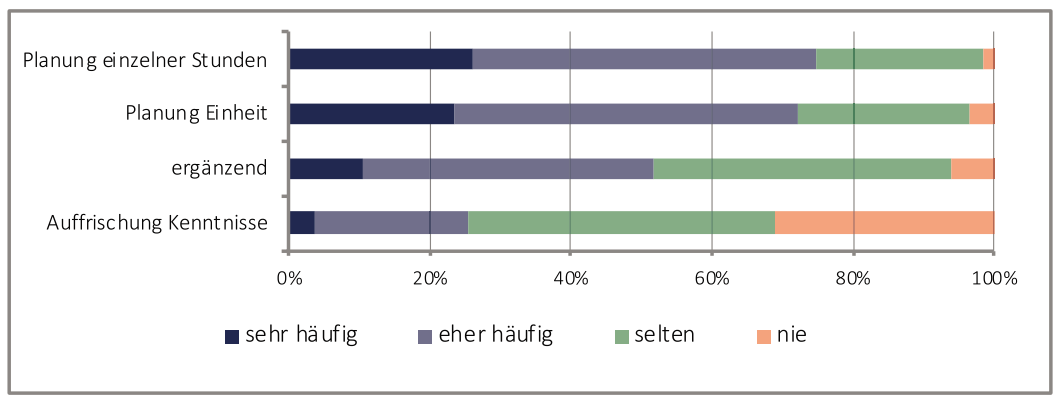

Abbildung 13: Nutzungsarten des EU-Kapitels (Lehrkräfte, $n=123$ )

Ebenfalls wurde nach den einzelnen Bestandteilen des EU-Kapitels und deren Verwendungshäufigkeiten im Unterricht gefragt. Die unten abgebildete Darstellung (vgl. Abbildung 14) zeigt die Rangfolge der eingesetzten Kapitelbestandteile. Die Sortierung erfolgt unter Orientierung an den ersten beiden Antwortoptionen (sehr häufig und eher häufig). Es zeigt sich, dass mehr als $90 \%$ der Lehrerinnen und Lehrer Karikaturen, Schaubilder und Grafiken sehr häufig oder eher häufig einsetzen. Weniger als die Hälfte nutzen z.B. die Arbeitsaufträge und Methodenvorschläge im Kapitel. Die geringsten Angaben wurden zur Nutzung des Glossars gemacht; dies ist aber insofern schwierig zu bewerten, als nicht alle Schulbücher über ein solches Verzeichnis verfügen. Weiterhin interessant ist, dass nur wenige Lehrkräfte die genuine Eigenarbeit der Autorinnen und Autoren sehr häufig verwenden. So liegen die Werte für »sehr häufig« bei Zusammenfassungen, Autorentexten und Arbeitsaufträgen bei etwa $10 \%$ oder darunter.

Ob die Häufigkeit der Nutzung einzelner Bestandteile des Kapitels davon abhängt, welches Schulbuch den Lehrkräften zur Verfügung steht, ist - für die fünf in dieser Stichprobe am häufigsten vorkommenden Lehrwerke der folgenden Tabelle (vgl. Tabelle 1) zu entnehmen. Die Darstellung erfolgt aus Gründen der Übersichtlichkeit mit Mittelwerten ( $1=$ sehr häufig, 2 = eher häufig, 3 = selten, $4=$ nie). Es zeigen sich große Unterschiede zwischen Politik \& Co. (C.C. Buchner) bzw. Mensch \& Politik (Schroedel) 


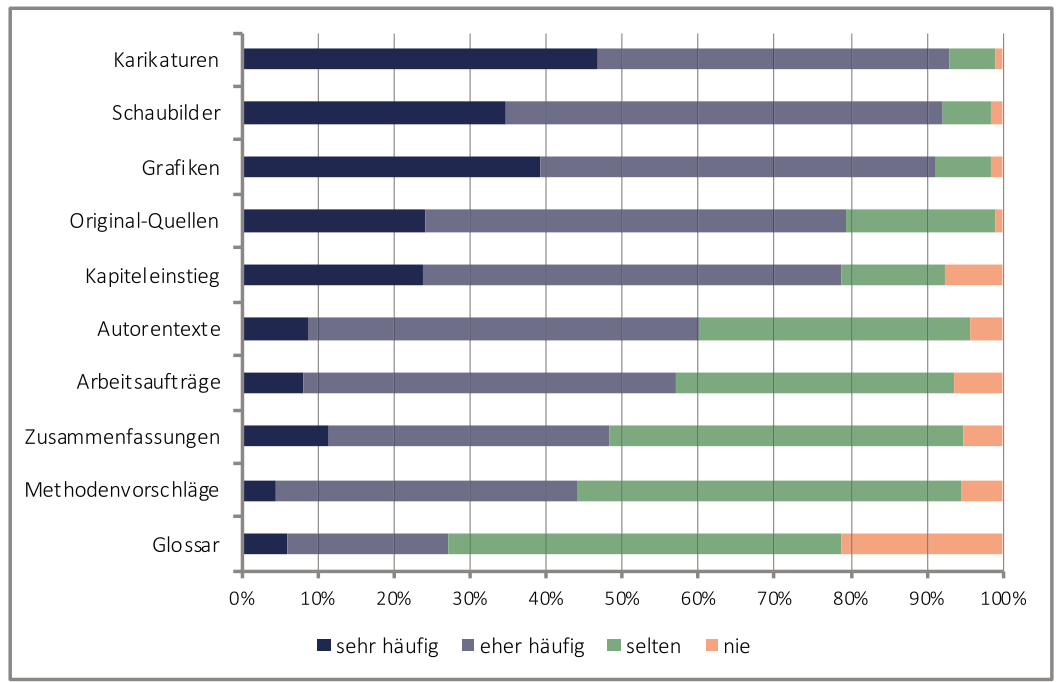

Abbildung 14: Nutzungshäufigkeit einzelner Kapitelbestandteile (Lehrkräfte, $n=123$ )

und z. B. Kompetenz Politik (Westermann) und Politik-Wirtschaft (Schöningh) im Hinblick auf die Nutzung von visuellen Inhalten wie Karikaturen, Schaubilder oder Grafiken. Auch hinsichtlich der Autorentexte, Arbeitsaufträge und Methodenvorschläge ist ein intensiverer Einsatz der Nutzerinnen und Nutzer von Politik \& Co. oder Mensch \& Politik festzustellen.

Tabelle 1: Nutzung von unterschiedlichen Kapitelbestandteilen in Abhängigkeit vom Politikschulbuch (Lehrkräfte, $\mathrm{n}=123$ )

\begin{tabular}{|l|l|l|l|l|l|}
\hline & $\begin{array}{l}\text { Politik \& } \\
\text { Co. }\end{array}$ & $\begin{array}{l}\text { Mensch \& } \\
\text { Politik }\end{array}$ & $\begin{array}{l}\text { Kompetenz } \\
\text { Politik }\end{array}$ & $\begin{array}{l}\text { Politik- } \\
\text { Wirtschaft }\end{array}$ & Anstöße 2 \\
\hline Karikaturen & $1,47(.60)$ & $1,50(.64)$ & $1,90(.57)$ & $2,00(.54)$ & $1,67(.58)$ \\
\hline Schaubilder & $1,69(.62)$ & $1,64(.68)$ & $1,80(.42)$ & $2,00(.54)$ & $2,00(.00)$ \\
\hline Grafiken & $1,62(.67)$ & $1,71(.72)$ & $1,80(.42)$ & $1,88(.64)$ & $2,00(.00)$ \\
\hline Original-Quellen & $1,96(.60)$ & $1,75(.75)$ & $2,20(.63)$ & $2,13(.84)$ & $2,50(.71)$ \\
\hline Kapiteleinstieg & $2,29(.88)$ & $2,39(.67)$ & $2,50(.71)$ & $2,50(.76)$ & $2,50(.71)$ \\
\hline Autorentexte & $2,67(.67)$ & $2,29(.76)$ & $2,40(.70)$ & $2,78(.97)$ & $3,00(.00)$ \\
\hline Arbeitsaufträge & $2,33(.74)$ & $2,36(.62)$ & $2,60(.84)$ & $2,88(.84)$ & $2,00(.00)$ \\
\hline
\end{tabular}


(Fortsetzung)

\begin{tabular}{|l|l|l|l|l|l|}
\hline & $\begin{array}{l}\text { Politik \& } \\
\text { Co. }\end{array}$ & $\begin{array}{l}\text { Mensch \& } \\
\text { Politik }\end{array}$ & $\begin{array}{l}\text { Kompetenz } \\
\text { Politik }\end{array}$ & $\begin{array}{l}\text { Politik- } \\
\text { Wirtschaft }\end{array}$ & Anstöße 2 \\
\hline Zusammenfassung & $2,40(.75)$ & $2,46(.79)$ & $2,50(.97)$ & $2,88(.64)$ & $2,50(.71)$ \\
\hline Methodenaufträge & $2,45(.69)$ & $2,78(.58)$ & $2,90(.74)$ & $2,85(.46)$ & $2,67(.58)$ \\
\hline Glossar & $2,81(.78)$ & $3,04(.74)$ & $2,80(.92)$ & $2,88(1.24)$ & - \\
\hline
\end{tabular}

Vierstufige Likert-Skala: 1 = sehr häufig, 4 = nie, Mittelwerte und Standardabweichung (in Klammern)

Für welche Unterrichtsphase der EU-Vermittlung bzw. zu welchem Zweck die Lehrkräfte das Kapitel nutzen, ist Abbildung 15 zu entnehmen. Insbesondere bei der Grundlagenvermittlung, aber auch bei den Institutionen und der Geschichte wird auf das Kapitel zurückgegriffen. Eher weniger wird es für die Erteilung von Hausaufgaben genutzt und der überwiegende Anteil der Lehrkräfte nutzt es selten oder nie zur Erstellung von Klassenarbeiten. Dennoch sagten immerhin ca. $15 \%$ der Befragten, dass sie das Politikschulbuch sehr häufig oder eher häufig dafür nutzen.

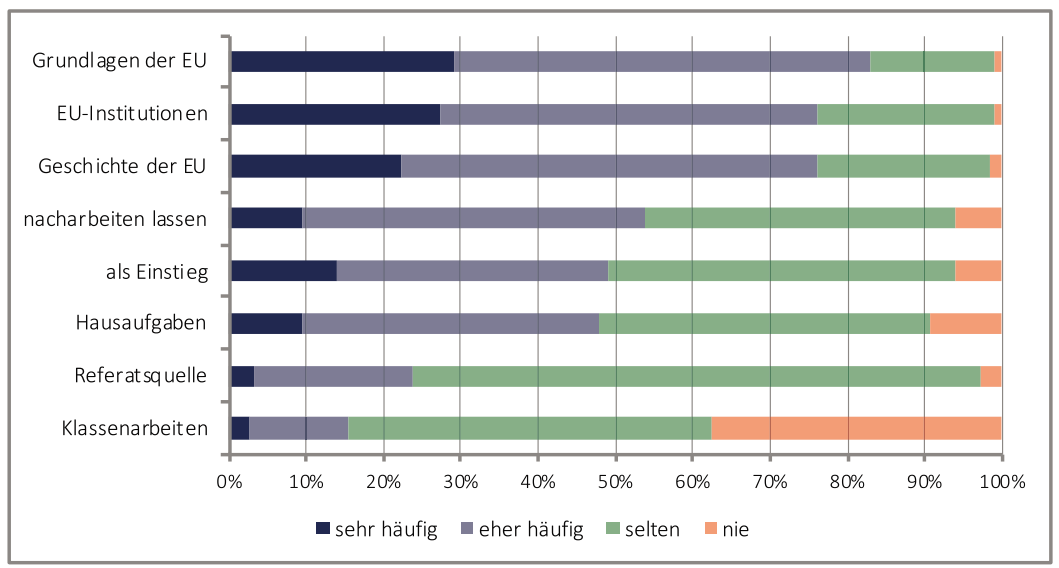

Abbildung 15: Zweck der EU-Kapitel-Nutzung (Lehrkräfte, $\mathrm{n}=123$ )

Aus den verschiedenen Angaben zur Nutzung lassen sich drei Nutzungstypen in Bezug auf das EU-Kapitel voneinander abgrenzen: erstens die Nutzung des Schulbuchs als Leitmedium, zweitens die Nutzung zur regel- 
mäßigen Orientierung (mittlere Nutzung) sowie drittens die Nutzung als sogenannter Steinbruch (gemeint ist eine eher ergänzende Verwendung; vgl. Rauch und Tomaschewski 1986). Dabei kann festgestellt werden, dass fachfremd unterrichtende Lehrkräfte signifikant häufiger Nutzungstyp 1 (Leitmedium) zuzuordnen sind und ältere Lehrkräfte sowie Gymnasiallehrkräfte signifikant seltener im Nutzungstyp 3 (Steinbruch) vorkommen (vgl. hierzu Tatje 2017). Im anschließenden Nutzungsmodell kann zwischen vier Dimensionen der Nutzung differenziert werden (Planung und Vermittlung von Grundlagen [1], Einsatz von Abbildungen und Texten [2], Verwendung von Arbeitsaufträgen und Zusammenfassungen [3] sowie Hausaufgaben [4]). Ältere Lehrkräfte nutzen es häufiger, um Hausaufgaben aufzugeben und Inhalte nacharbeiten zu lassen; am Gymnasium wird deutlich häufiger mit dem Schulbuch geplant und hier werden auch die darin angebotenen Materialien häufiger genutzt. Lehrkräfte, die über sich selbst sagen, viel über die EU zu wissen, setzen diese Materialien eher ein. Personen, die von ihrer Selbstwirksamkeit im EU-Unterricht überzeugt sind, benötigen das Buch hingegen weniger für die generelle Planung und Vermittlung der Grundlagen. Tendenziell reflektieren die Lehrkräfte die Qualität des Buches und nutzten positiv beurteile Bücher häufiger.

Im Rahmen dieser Teilstudie galt es weiterhin, eine Vorstellung von der Gestaltung des EU-Unterrichts insgesamt zu bekommen. So wurde nach weiteren - d.h. neben dem Politikschulbuch eingesetzten - Materialien und Medien gefragt und verglichen, wie gerne die Lehrkräfte mit diesen arbeiten. Die Schülerinnen und Schüler wurden darüber hinaus explizit nach den von ihnen favorisierten Materialien und Medien gefragt und wie häufig sie diese für die Vorbereitung des Unterrichts zur EU nutzen.

Zunächst folgt eine Darstellung der von Lehrkräften angegebenen Häufigkeiten zum Einsatz ausgewählter Materialien, die nicht aus dem aktuell genutzten Politikschulbuch entnommen werden (vgl. Abbildung 16). Diese zeigt, dass grundsätzlich visuelle Materialien wie Grafiken, Schaubilder und Karikaturen sehr beliebt sind - jeweils mehr als $90 \%$ der Lehrkräfte nutzen diese sehr häufig oder eher häufig. Doch auch aktuelles Material wird von ca. $93 \%$ regelmäßig genutzt. Arbeitsblätter (90\%) und Folien bzw. Tafelbilder (88 \%) spielen nach wie vor eine wichtige Rolle im Unterricht zur EU. Videomaterial, das Internet generell und digitale Medien wie CD-ROMs werden deutlich seltener - von einigen sogar nie - benutzt. 


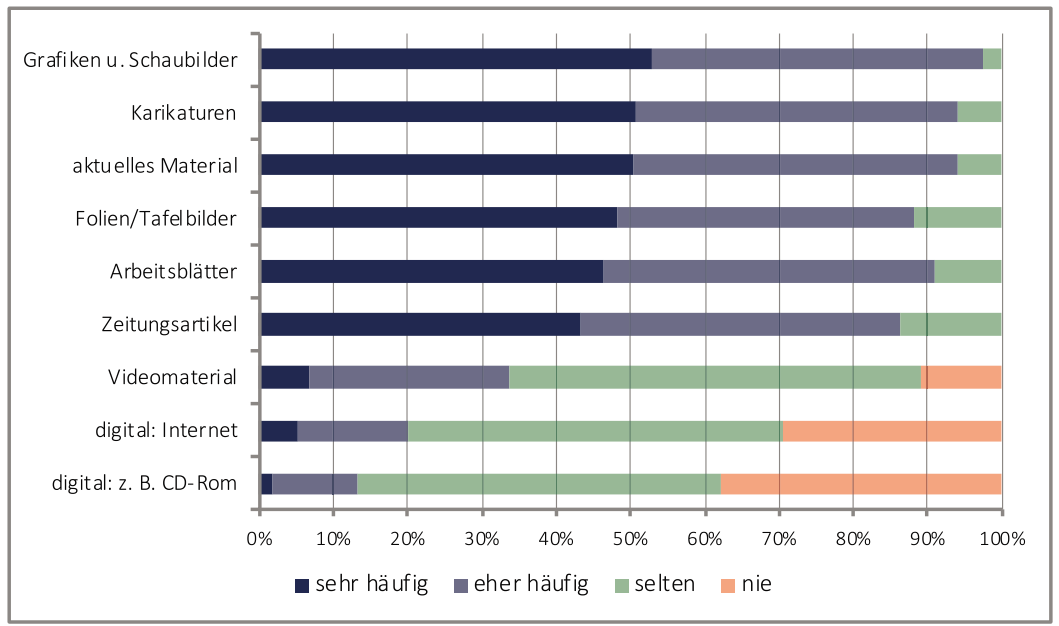

Abbildung 16: Im EU-Unterricht eingesetzte Materialien, die nicht dem Buch entnommen wurden (Lehrkräfte, $\mathrm{n}=123$ )

Zusätzlich wurde erhoben, mit welchen der eingesetzten Materialien am liebsten im EU-Unterricht gearbeitet wird (vgl. Abbildung 17). Dabei stellt sich heraus, dass 45,8 \% der Lehrkräfte Karikaturen allen anderen Materialien vorziehen. Erst mit deutlichem Abstand folgen Arbeitsblätter (35,2 \%), Zeitungsartikel (34,2 \%) und aktuelles Material (33,4 \%). Obwohl das Schulbuch, wie oben gezeigt, von einer großen Mehrheit der Lehrkräfte häufig eingesetzt wird, geben nur $22,9 \%$ an, es im Unterricht zur EU am liebsten zu benutzen. Mit 19,6 \% folgen Grafiken und Schaubilder, die vermutlich i.d.R. aus dem Schulbuch entnommen werden. Digitale Materialien $(9,0 \%)$ und Videos (6,5\%) finden eher wenig Zustimmung; mit CDROMs arbeitet niemand am liebsten. Diese Angaben der Lehrkräfte sind besonders interessant, wenn sie mit den Angaben der Schülerinnen und Schüler verglichen werden. In der Studie wurden im Lehrer- wie auch im Schülerfragebogen dieselben Batterien und Formulierungen der Fragen zu den eingesetzten Materialien genutzt. Dabei kann festgestellt werden, dass es eine erhebliche Diskrepanz in der Vorliebe für Videomaterial, Grafiken und Schaubilder (wie auch Folien bzw. Tafelbilder) gibt. Hier geben deutlich mehr Lernende an, diese Materialien am liebsten im EU-Unterricht zu nutzen. Umgekehrt schätzen Lehrkräfte ihre Arbeitsblätter, Zeitungsartikel 
und generell aktuelles Material deutlich mehr als Schülerinnen und Schüler. Für das Schulbuch zeigt sich zwar insgesamt eine eher geringe Wertschätzung, doch weichen die Angaben beider Gruppen nicht so stark voneinander $\mathrm{ab}$ wie bei anderen Materialien.

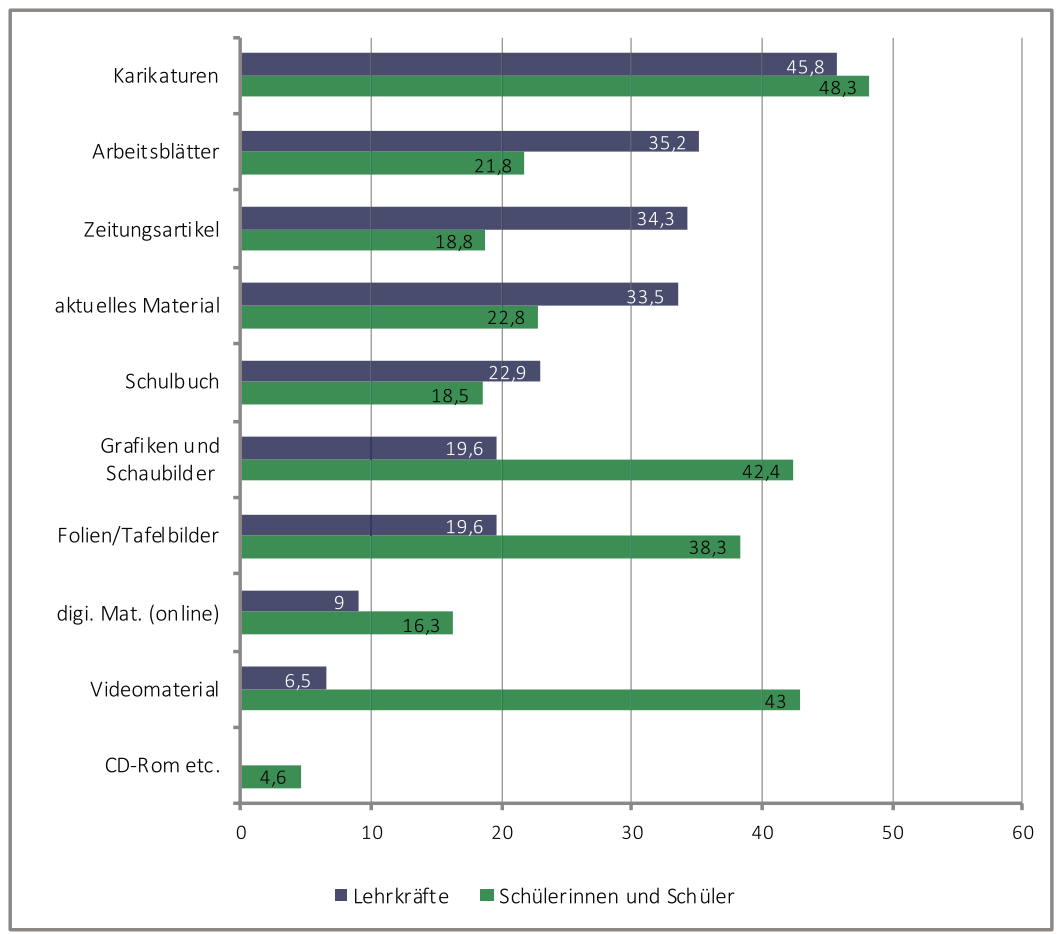

Abbildung 17: Vergleich Lieblingsmaterial im EU-Unterricht (Lehrkräfte vs. Schülerinnen und Schüler)

Die Schülerinnen und Schüler wurden außerdem gefragt, welche Materialien oder Medien sie wie häufig zur Vorbereitung auf den EU-Unterricht verwenden. Die deskriptive Auswertung zeigt unter anderem, dass Nachrichten im Fernsehen sowie das Internet (Suchmaschinen und Lexika) von mehr als 50 \% regelmäßig genutzt werden, während das Politikschulbuch immerhin noch von ca. $30 \%$ der Lernenden zur Vorbereitung eingesetzt wird (vgl. Abbildung 18). 


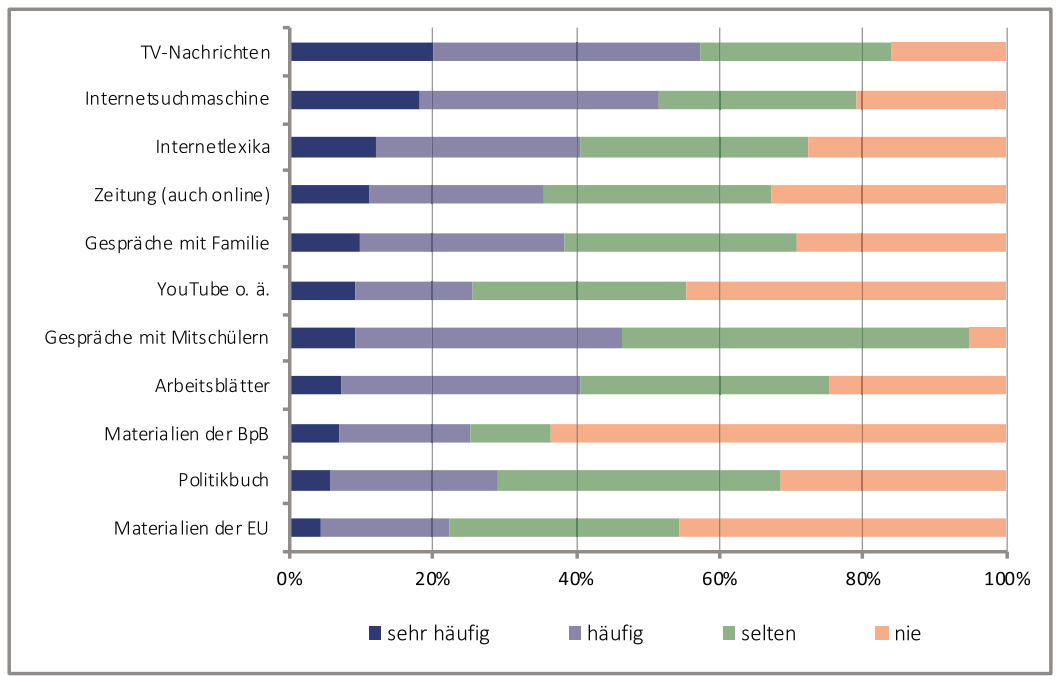

Abbildung 18: Eingesetzte Materialien und Medien zur Vorbereitung auf den EU-Unterricht (Schülerinnen und Schüler, $\mathrm{n}=1076$ )

Das folgende Balkendiagramm zeigt auf, welche weiteren Materialien neben dem Schulbuch im Unterricht zur EU eingesetzt werden (vgl. Abbildung 19). Den höchsten Wert (ca. 90 \% bei »sehr häufig" und »eher häufig«) erreichten hier die Zeitungsartikel, was mit der oben bereits vorgestellten Beliebtheit von aktuellem Material einhergeht. Ebenfalls recht häufig werden Materialien der Bundeszentrale für politische Bildung (BpB), der EU selbst sowie die Praxis-Politik-Hefte eingesetzt. Im digitalen Bereich werden (neben der $\mathrm{BpB}$ ) die Internetseiten der EU(-Institutionen) mit ca. 50 \% sehr häufig oder eher häufig angegeben; auch die Internetseiten der öffentlichen Fernsehsender (z.B. ARD.de) scheinen zumindest von einem Teil der Lehrkräfte regelmäßig genutzt zu werden.

In der Schülerstudie konnte das generelle Politikschulbuch-Nutzungsverhalten der Lernenden als sechs-faktorielles Konstrukt modelliert werden, mit gutem Modellfit $\left(x^{2}=511.33(194)^{\star * *}\right.$, CFI/TLI $=.98 / .97$, RMSEA $=.03$, WRMR $=1.31$ ); für einen Überblick der Dimensionen sowie der dazugehörigen Beispielitems und Cronbach's Alpha ${ }^{12}$ siehe Abbil-

12 Cronbach's Alpha $(\alpha)$ ist ein Maß für die interne Konsistenz einer Skala aus mehreren 


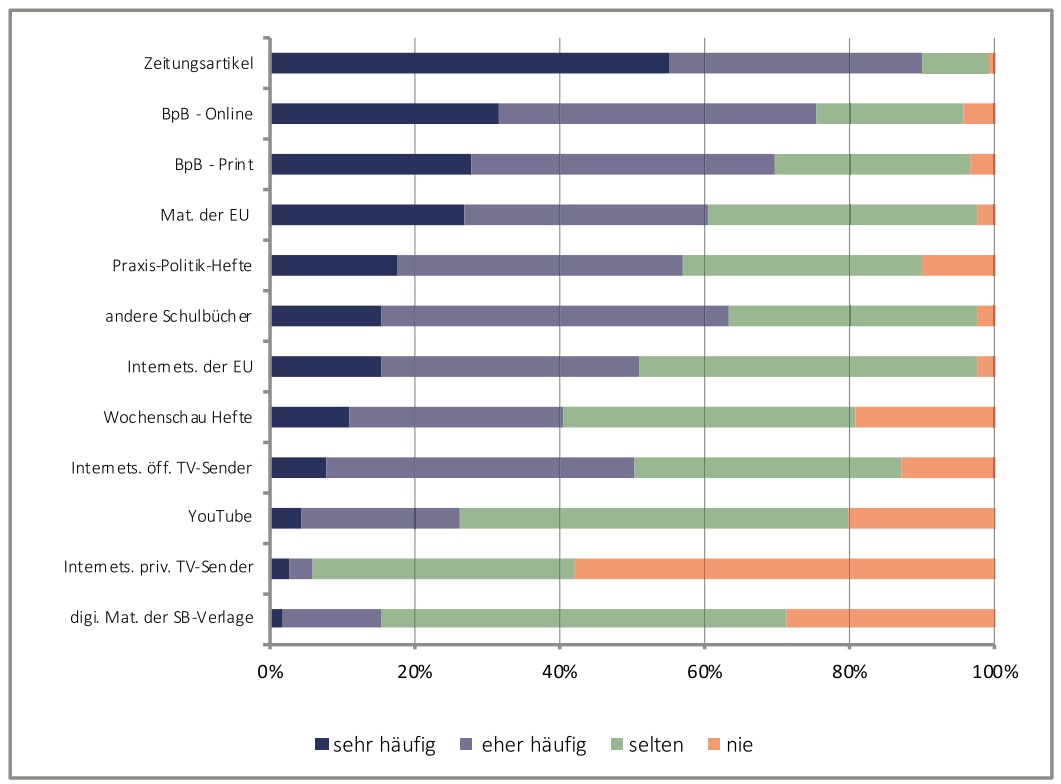

Abbildung 19: Weitere genutzte Materialien und Medien im EU-Unterricht (Lehrkräfte, $\mathrm{n}=123$ )

dung 20 und Tabelle 2. Die durchweg höchst signifikanten Korrelationen der Facetten untereinander liegen zwischen $r=.22$ und $r=.72$. Ein Vorläufermodell verfügte über eine siebte Facette »unkonventionelle Nutzung des Schulbuchs im Unterricht « (z. B. »um mein Handy zu verstecken« oder "um der Lehrkraft vorzuspielen, dass ich aufpasse $).{ }^{13}$ Allerdings war der Modellfit dem hier vorgestellten sechs-faktoriellen Modell unterlegen und die Facette "unkonventionelle Nutzung" teilweise nicht mit den übrigen Facetten korreliert. Die »unkonventionelle Nutzung» findet im Folgenden daher keine weitere Berücksichtigung.

Items und gibt an, wie zuverlässig (reliabel) diese Items ein eindimensionales Konstrukt erfassen. Werte ab 0,7 gelten als akzeptabel, ab 0,8 spricht man von einer hohen Reliabilität.

$1312,7 \%$ der Schülerinnen und Schüler gaben an, mit dem Schulbuch sehr häufig oder häufig ihr Handy zu verstecken. 26,7 \% nutzen das Politikschulbuch sehr häufig oder häufig, um der Lehrkraft Konzentration vorzuspielen. Mädchen äußerten ein geringeres unkonventionelles Nutzungsverhalten als Jungen. 


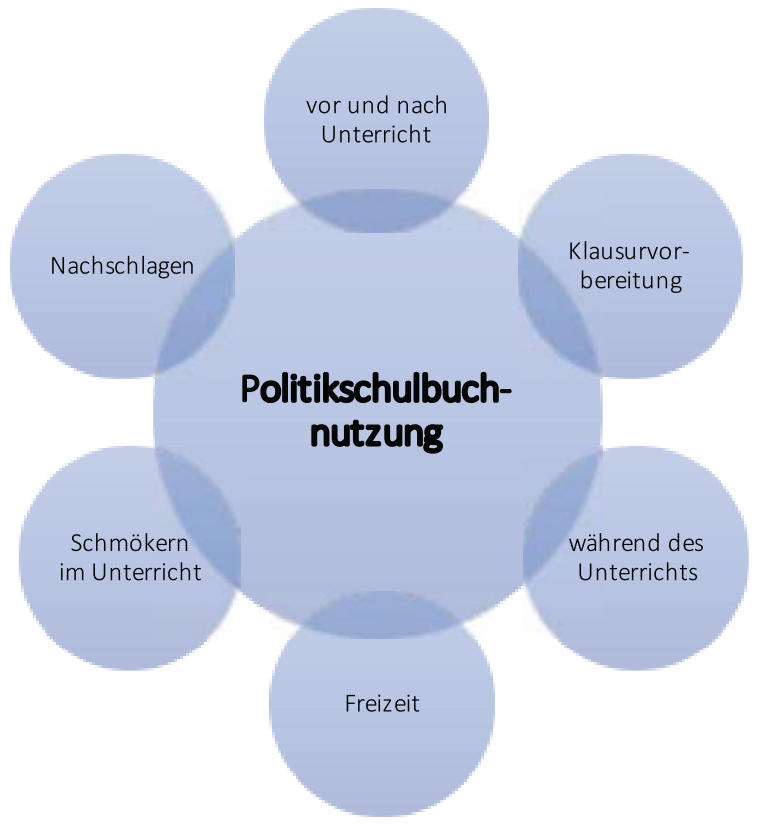

Abbildung 20: Sechs-faktorielles Schulbuchnutzungs-Modell (Schülerinnen und Schüler, $\mathrm{n}=1076)$

Einen Überblick über das Nutzungsverhalten der Schülerinnen und Schüler geben die Mittelwerte (M) und Standardabweichungen (SD) der einzelnen Dimensionen in Tabelle 2. Im Durchschnitt setzen die Schülerinnen und Schüler ihr Politikschulbuch am häufigsten während des Unterrichts $(M=2,17)$ und zum Lernen vor einer Klausur ein $(M=2,39)$, außerdem zum Nachschlagen von Begriffen $(M=2,81)$. Eher selten wird es zur Unterrichtsvor- und -nachbereitung eingesetzt $(M=3,37)$ sowie zum Schmökern im Unterricht $(M=3,11)$. Eine Nutzung des Politikschulbuchs in der Freizeit kommt vor, erwartungsgemäß allerdings selten $(M=3,69)$. 
Tabelle 2: Faktoren der Schulbuchnutzung von Schülerinnen und Schülern: Cronbach's Alpha, Mittelwerte, Standardabweichung (in Klammern) und Beispielitems

\begin{tabular}{|l|l|l|l|l|}
\hline Faktor & $\begin{array}{l}\text { Item- } \\
\text { Anzahl }\end{array}$ & $\boldsymbol{\alpha}$ & $\begin{array}{l}\text { M } \\
(\text { SD })\end{array}$ & $\begin{array}{l}\text { Beispielitem } \\
\text { »ch nutze das Politikschulbuch, } \\
\text { um ... }\end{array}$ \\
\hline $\begin{array}{l}\text { vor/nach } \\
\text { Unterricht }\end{array}$ & 7 & .88 & $\begin{array}{l}3.37 \\
(.56)\end{array}$ & $\begin{array}{l}\text { mich kurz in das Thema der Stunde } \\
\text { einzulesen.» }\end{array}$ \\
\hline Klausurvorbereitung & 4 & .89 & $\begin{array}{l}2.39 \\
(.71)\end{array}$ & $\begin{array}{l}\text { wichtige Inhalte auswendig zu } \\
\text { lernen.» }\end{array}$ \\
\hline $\begin{array}{l}\text { während des } \\
\text { Unterrichts }\end{array}$ & 4 & .77 & $\begin{array}{l}2.17 \\
(.72)\end{array}$ & $\begin{array}{l}\text { mit der Klasse Buchinhalte zu } \\
\text { besprechen.» }\end{array}$ \\
\hline $\begin{array}{l}\text { Schmökern im } \\
\text { Unterricht }\end{array}$ & 3 & .70 & $\begin{array}{l}3.11 \\
(.65)\end{array}$ & $\begin{array}{l}\text { mich zu beschäftigen, weil der } \\
\text { Unterricht langweilig ist.» }\end{array}$ \\
\hline Nachschlagen & 2 & .67 & $\begin{array}{l}2.81 \\
(.77)\end{array}$ & Fachbegriffe nachzuschlagen.» \\
\hline Freizeit & 4 & .72 & $\begin{array}{l}3.69 \\
(.50)\end{array}$ & $\begin{array}{l}\text { in der Freizeit Karikaturen } \\
\text { anzusehen.» }\end{array}$ \\
\hline
\end{tabular}

Vierstufige Likert-Skala: 1 = sehr häufig, 2 = eher häufig, 3 = selten, $4=$ nie

Latente multiple Regressionen (vgl. Tabelle 3) zeigen den Einfluss verschiedener Schülerdispositionen auf die sechs Facetten der Schulbuchnutzung. Hier fällt zum einen auf, dass Schülerinnen es häufiger in lernrelevanten Kontexten (z.B. zur Klausurvorbereitung) nutzen und weniger zum Schmökern (aus Langerweile im Unterricht) als ihre männlichen Mitschüler. Zum anderen wirkt sich der Migrationshintergrund - unter Kontrolle des kulturellen Kapitals (Proxy-Indikator: Bücherfrage) - positiv auf die Nutzung des Politikschulbuchs zur Vor- und Nachbereitung des Unterrichts sowie zum Nachschlagen von Fachbegriffen oder Fremdwörtern aus. Auch in der Freizeit nutzen Schülerinnen und Schüler mit Migrationshintergrund das Politikschulbuch signifikant häufiger. 
Tabelle 3: Latente multiple Regression zur Politikschulbuch-Nutzung von Schülerinnen und Schülern

\begin{tabular}{|l|l|l|l|l|l|l|}
\hline & $\begin{array}{l}\text { vor/ } \\
\text { nach } \\
\text { PU }\end{array}$ & $\begin{array}{l}\text { vor } \\
\text { Klausur }\end{array}$ & $\begin{array}{l}\text { während } \\
\text { des PU }\end{array}$ & Freizeit & „Schmökern“ & Nachschlagen \\
\hline $\begin{array}{l}\text { Geschlecht } \\
(\mathrm{w})\end{array}$ & $.20^{\star}$ & $.42^{* * *}$ & $\mathrm{~ns}$ & $\mathrm{~ns}$ & $-.49^{* * *}$ & $.31^{\star * \star}$ \\
\hline Alter & $.09^{\star}$ & $\mathrm{ns}$ & $\mathrm{ns}$ & $.09^{\star}$ & $\mathrm{ns}$ & $.08^{\star}$ \\
\hline $\begin{array}{l}\text { kult. } \\
\text { Kapital }\end{array}$ & $\mathrm{ns}$ & $\mathrm{ns}$ & $\mathrm{ns}$ & $.08^{\star}$ & $.12^{\star *}$ & $.09^{\star}$ \\
\hline Migration & $.43^{* * \star}$ & $\mathrm{ns}$ & $\mathrm{ns}$ & $.19^{\star}$ & $\mathrm{ns}$ & $.30^{\star *}$ \\
\hline $\mathrm{R}^{2}$ & .05 & .05 & .01 & .02 & .07 & .06 \\
\hline
\end{tabular}

${ }^{* * *} \mathrm{p} \leq .001,{ }^{* *} \mathrm{p} \leq .01,{ }^{*} \mathrm{p} \leq .05 ; \mathrm{R}^{2}=$ Anteil der aufgeklärten Varianz; Koeffizienten im Falle kontinuierlicher Prädiktoren standardisiert, für dichotome Prädiktoren (Geschlecht, Migration) unstandardisiert

Für die Lehrkräfte hat das Schulbuch insbesondere im Kontext der Vorbereitung des politischen Fachunterrichts eine hohe Bedeutung: 26,2 \% empfinden das Buch dabei als sehr wichtig, 43,4 \% als eher wichtig, 28,7 \% als eher unwichtig und nur 1,6 \% als sehr unwichtig. Während des Unterrichts messen die Politiklehrerinnen und -lehrer dem Schulbuch eine etwas geringere, aber noch immer hohe Bedeutung bei: 20,5\% empfinden es dabei als sehr wichtig, 47,5\% als eher wichtig, 30,3\% als eher unwichtig und wiederum 1,6\% als sehr unwichtig. Für vertiefende Analysen und weitere latent modellierte Modelle zur Nutzung der Politikschulbücher bzw. der EU-bezogenen Kapitel aus Schüler- und Lehrerperspektive vgl. Tatje (2017).

\section{III.4 Ergebnisse zur Bewertung}

Das unten abgebildete Balkendiagramm (vgl. Abbildung 21) zeigt die generelle Zufriedenheit der Lehrkräfte mit dem Politikschulbuch insgesamt sowie dem EU-Kapitel des jeweiligen Buches im Speziellen. So lassen sich die einzelnen Ergebnisse sinnvoller einordnen. Mithilfe der vierstufigen Antwortoptionen "sehr zufrieden «, "eher zufrieden «, »eher unzufrieden" und "sehr unzufrieden« ergibt sich ein erster Eindruck von der Beurteilung der Schulbücher aus Lehrerperspektive. Über die höchsten Mittelwerte verfügt 
Votum (Schöningh); hier ist jedoch anzumerken, dass lediglich eine Lehrkraft dieses - zum Zeitpunkt der Erhebung neu erschienene - Werk genutzt hat. Es folgen Mensch \& Politik (Schroedel) sowie Politik \& Co. (C.C. Buchner), während Kompetenz Politik (Westermann) und Politik-Wirtschaft (Schöningh) im Gesamteindruck am schlechtesten abschneiden. Auffällig sind die bei fast allen Schulbüchern leicht negativeren Mittelwerte der $\mathrm{Zu}$ friedenheit mit dem EU-Kapitel im Vergleich zum Schulbuch insgesamt. Nur beim Lehrwerk Politik-Wirtschaft schneidet das EU-Kapitel etwas besser ab $(M=3,13)$ als das Gesamtwerk $(M=3,22)$, wobei anzumerken ist, dass hier beide Werte jeweils das schlechteste Bewertungsergebnis im Vergleich zu den anderen Büchern darstellen.

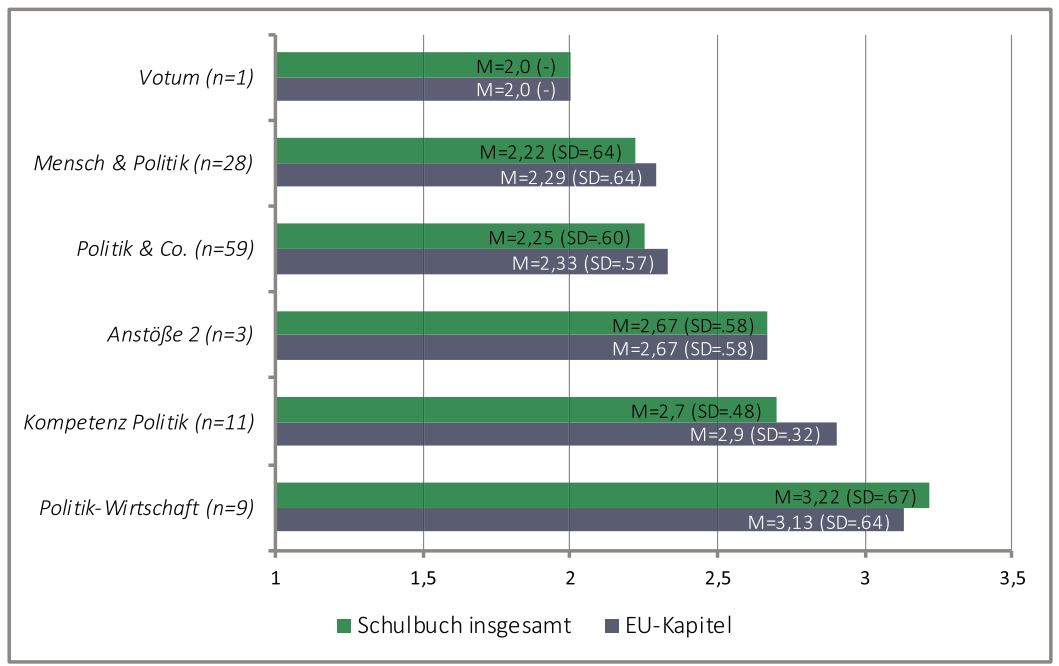

Abbildung 21: Zufriedenheit Lehrkräfte: Schulbuch und EU-Kapitel; Mittelwert (M) und Standardabweichung (SD), 1 = sehr zufrieden, $4=$ sehr unzufrieden

Ergänzt werden können die Angaben der Lehrkräfte durch den abgefragten Wunsch, lieber mit einem anderen Schulbuch im Politikunterricht zu arbeiten. Während bei Votum kein Wunsch nach einem Austausch besteht, wollen bei Politik \& Co. (21,3 \%) sowie Mensch \& Politik (25,9\%) nur wenige Personen tauschen. Anders verhält es sich bei Nutzerinnen und Nutzern von Anstöße 2 (50,0 \%) Kompetenz Politik (mehr als $66 \%$ ) und von 
Politik - Wirtschaft (mehr als $83 \%$ ), die lieber mit einem anderen Lehrwerk arbeiten möchten. Mit welchem Buch die Lehrkräfte stattdessen lieber arbeiten würden, haben sie allerdings nicht angegeben. Für einen Vergleich von Schüler- und Lehrerangaben folgt nun die Gegenüberstellung der Ergebnisse der Mittelwerte aus Schülerperspektive.

Auch am folgenden Balkendiagramm (vgl. Abbildung 22) zur Beurteilung aus Schülerperspektive wird deutlich, dass Votum (hier einschränkend die geringe Schülerzahl von $n=18$ ) die höchsten Mittelwerte für das Buch insgesamt $(M=2,12)$ wie auch für das EU-Kapitel $(M=2,33)$ aufweist, wenn auch etwas schwächer als bei der dazugehörigen Lehrkraft. Die Rangfolge stimmt weitgehend mit der Einschätzung der Lehrkräfte überein, wobei die Schülerinnen und Schüler Kompetenz Politik am schlechtesten bewerten und Politik-Wirtschaft etwas besser beurteilen. Die Schülerinnen und Schüler sehen jedoch größere Unterschiede zwischen dem Politikschulbuch insgesamt und dem EU-Kapitel, welches schulbuchübergreifend besser bewertet wird als das Buch.

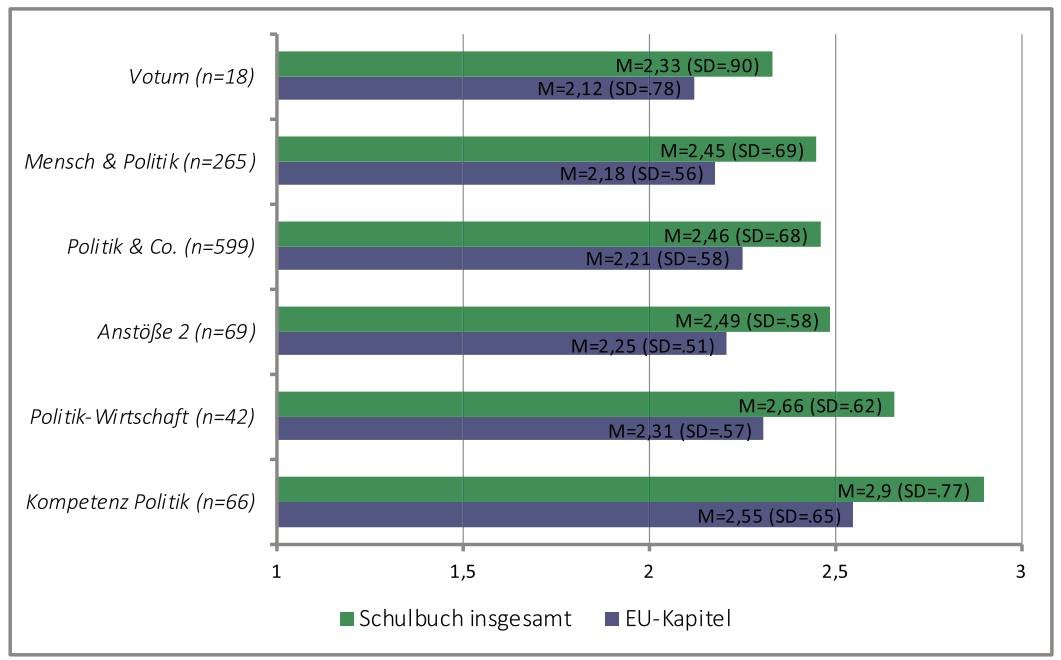

Abbildung 22: Zufriedenheit Schülerinnen und Schüler: Schulbuch und EU-Kapitel; Mittelwert (M) und Standardabweichung (SD), $1=$ sehr zufrieden, $4=$ sehr unzufrieden 


\section{III.5 Detaillierte Bewertung des EU-Kapitels (Lehrkräfte)}

Eine detaillierte Beurteilung des EU-Kapitels durch Lehrkräfte ist Abbildung $23 \mathrm{zu}$ entnehmen. Mithilfe von vier Antwortoptionen (sehr gut, eher gut, eher schlecht, sehr schlecht) konnten Aspekte des Kapitels bewertet werden. Auffällig ist zunächst, dass es nur recht wenige Lehrkräfte gibt, die die Bestandteile bzw. Eigenschaften des Kapitels mit sehr gut bewerten (nur bei Korrektheit sowie bei Grafiken und Schaubildern wird die $10 \%$-Marke überschritten). Insgesamt liegen diese beiden Facetten unter Hinzunahme der Kategorie »eher gut « allerdings bei fast $90 \%$; lediglich eine Lehrkraft empfindet die Korrektheit als sehr schlecht. Diese Person nutzt Gesellschaft bewusst (Westermann). Zwischen ca. $70 \%$ und $80 \%$ der Lehrkräfte bewerten die Geschichte und die Darstellung der Grundlagen der EU, die Kohärenz zwischen den Texten, Grafiken und Aufgabenstellungen sowie den Aufbau als sehr gut oder eher gut. Methodenvorschläge, Schülerorientierung, Aktualität, Thematisierung der Europawahlen und ferner der Lebensweltbezug werden tendenziell negativer beurteilt (d.h. von mindestens der Hälfte der Teilnehmerinnen und Teilnehmer als eher schlecht oder sehr schlecht bewertet). Der Bezug zur Lebenswelt wird als einziger Aspekt von keiner Person als sehr gut bezeichnet: über $60 \%$ schätzen dieses Attribut als eher schlecht oder sogar sehr schlecht ein. Der mit Abstand höchste Wert (ca. $23 \%$ ) bei der Antwortoption »sehr schlecht« kann hinsichtlich der Aktualität der EU-Kapitel gemessen werden. Unter Hinzunahme der Option »eher schlecht « empfinden etwa $65 \%$ der Lehrkräfte die (mangelnde) Aktualität als Schwäche der EU-Kapitel. Vergleichbar negative Werte sind auch in Bezug auf die Thematisierung der Europawahlen, den Lebensweltbezug und die Schülerorientierung festzustellen. Bemerkenswert ist, dass transmissive lehr-lern-theoretische Überzeugungen der Lehrkräfte nicht nur zu einer häufigeren Nutzung, sondern auch zu einer positiveren Bewertung des verwendeten EU-Kapitels führen. Stärker konstruktivistische lehr-lern-theoretische Überzeugungen gehen dagegen mit einer kritischeren Bewertung der Schülerorientierung des EU-Kapitels einher. 


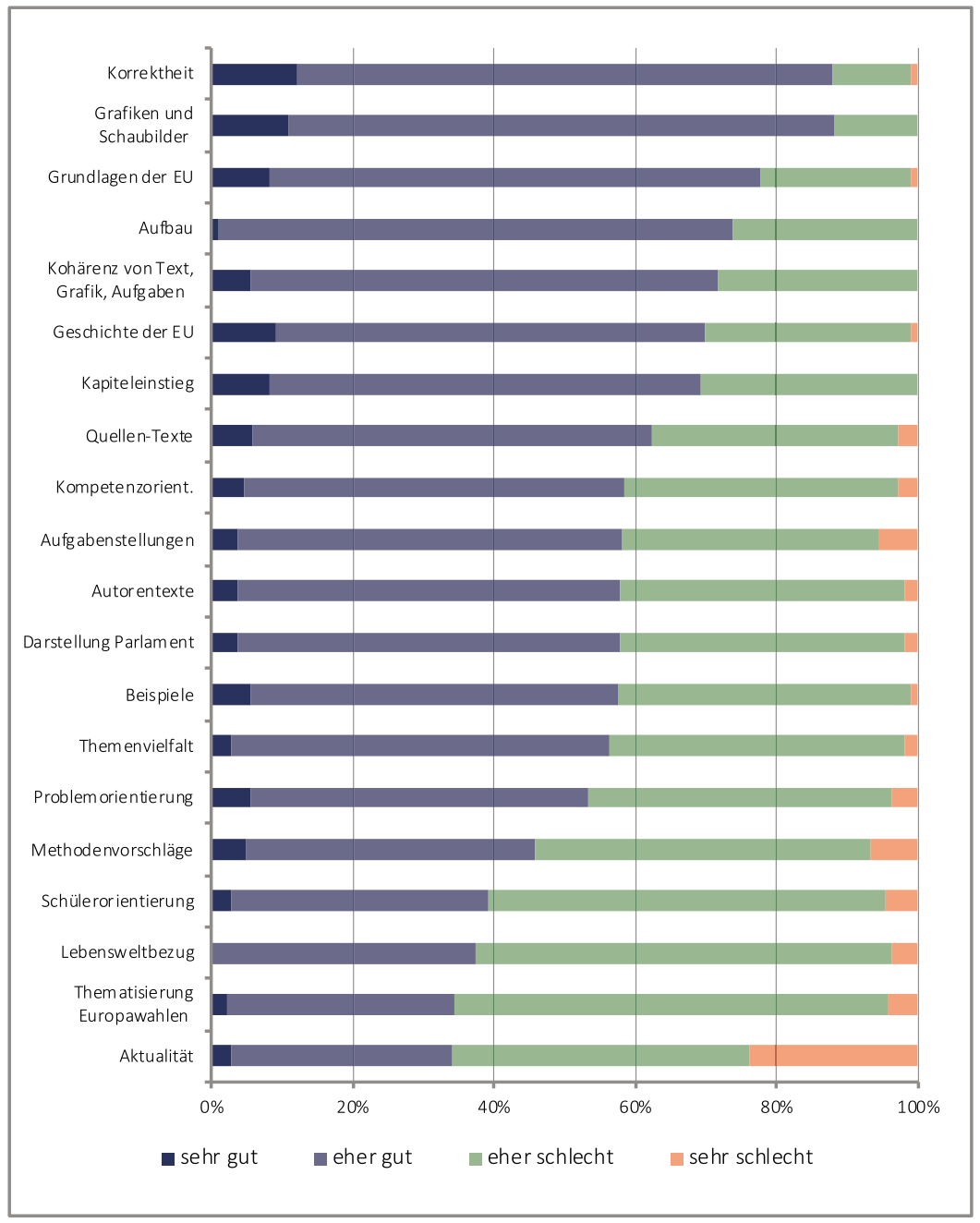

Abbildung 23: Beurteilung einzelner Aspekte im EU-Kapitel (Lehrkräfte, $n=123$ ) 


\section{III.6 Detaillierte Beurteilung des EU-Kapitels (Schülerinnen und Schüler)}

Auch die Schülerinnen und Schüler wurden detailliert zu ihren Meinungen zum Politikschulbuch befragt. In der folgenden Abbildung 24 sind zunächst allgemeine (positiv formulierte) Attribute des EU-Kapitels benannt. Aus Gründen des an die Bedürfnisse der Schülerinnen und Schüler angepassten Fragebogendesigns weichen die Formulierungen geringfügig von den Batterien im Lehrerfragebogen ab; die vier Antwortoptionen lauten hier "trifft voll und ganz zu «, "trifft eher zu «, »trifft eher nicht zu « und »trifft überhaupt nicht zu«. Zwischen $80 \%$ und $90 \%$ der befragten Lernenden beschreiben die EU-Kapitel als durchaus verständlich, übersichtlich und unkompliziert allerdings empfanden nur ca. $20 \%$ diese Aussagen als voll und ganz zutreffend. Zwischen $60 \%$ und $70 \%$ der Schülerinnen und Schüler stimmen der Aussage $\mathrm{zu}$, dass ihr EU-Kapitel ansprechend und auch interessant gestaltet ist, nicht zu viele Informationen enthält und eine gute Unterstützung für den Unterricht zur EU bietet (ca. 10-15 \% finden diese Attribute voll und ganz zutreffend). Weniger Zustimmung erfahren die Beschreibungen der Schulbücher als »abwechslungsreich", "hilfreich" und »spannend « - weniger als $10 \%$ empfinden dies als voll und ganz zutreffend. Lediglich $2 \%$ antworten auf die Aussage, das Kapitel sei auf das Leben der Schülerinnen und Schüler abgestimmt, mit "trifft voll und ganz zu«, und mehr als $15 \%$ geben an, dass dies ihrer Ansicht nach überhaupt nicht zutreffe.

Die Aufgabenstellungen im EU-Kapitel werden von mehr als der Hälfte der befragten Schülerinnen und Schüler als wenig kreativ, langweilig und nicht besonders originell beurteilt. Dabei liegt der Anteil derjenigen, die dem voll und ganz zustimmten, bei jeweils ca. $10 \%$ oder darunter. Ein $\mathrm{zu}$ niedriges Anforderungsniveau der Aufgabenstellungen wird dagegen überwiegend nicht als Problem eingeschätzt (fast $80 \%$ entschieden sich hier für eine ablehnende Option). Möglicherweise ist dieser Befund eher gegenteilig zu interpretieren, und die Aufgaben werden tendenziell als zu fordernd respektive zu schwierig empfunden. (Für vertiefende Analysen und weitere Modelle zur Beurteilung der Politikschulbücher und der EUbezogenen Kapitel aus Schüler- und Lehrerperspektive vgl. Tatje 2017.) 


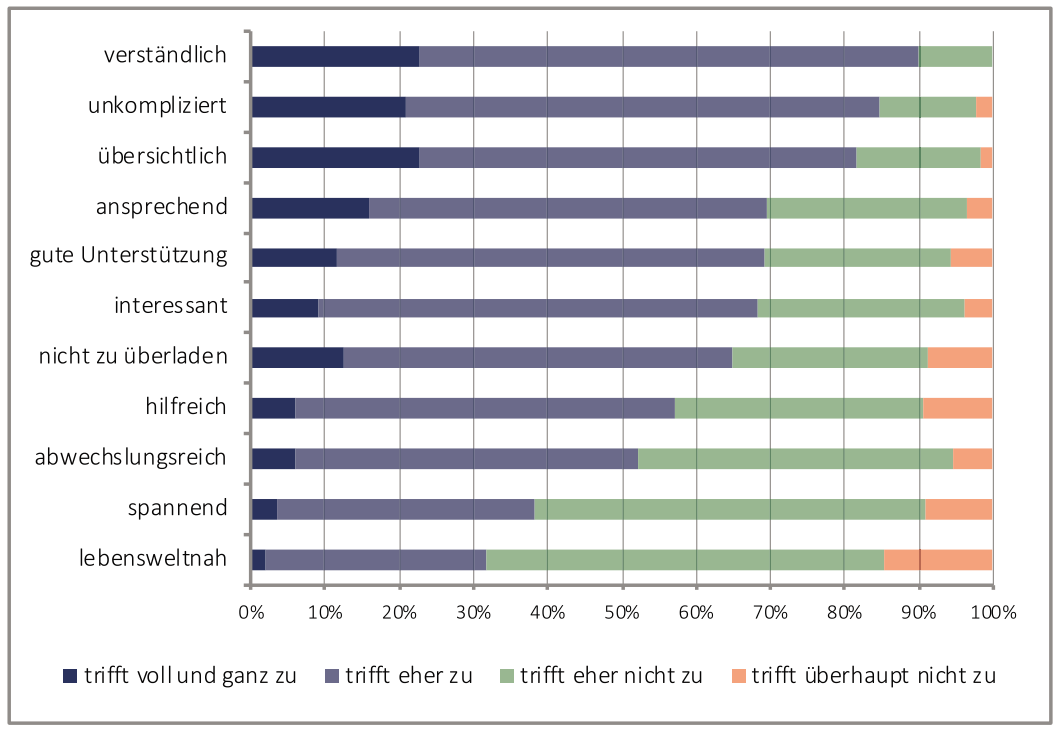

Abbildung 24: Beurteilung einzelner Aspekte im EU-Kapitel (Schülerinnen und Schüler, $\mathrm{n}=1076)$

\section{III.7 Zusammenfassung}

Zunächst lässt sich klar sagen, dass die Nutzung des Politikschulbuchs generell und im Unterricht zur EU intensiver stattfindet als vorab vermutetet. Keine der 52 befragten Klassen arbeitet ohne Politikschulbuch, und nur eine von 123 Politiklehrkräften gibt an, gänzlich auf das Schulbuch zu verzichten. Da die befragten Klassen und Lehrpersonen nicht nach ihrem SchulbuchNutzungsverhalten ausgewählt wurden, ist davon auszugehen, dass ein vollständiger Verzicht auf das Lehrwerk im Politikunterricht niedersächsischer Gymnasien und Gesamtschulen nur äußerst selten vorkommt. Das Lehrwerk insgesamt wie auch das EU-Kapitel werden insbesondere zur Planung von ganzen Einheiten, aber auch von einzelnen Unterrichtsstunden sowie zur Vermittlung der Grundlagen wie beispielsweise Institutionenkunde sehr häufig oder häufig verwendet. Es wird somit häufig als Leitmedium des Politikunterrichts eingesetzt. Dabei konnte festgestellt 
werden, dass besonders die angebotenen Materialien wie Karikaturen, Schaubilder oder Grafiken verwendet werden und weniger die Autorentexte oder Methodenvorschläge. Insbesondere fachfremde Lehrkräfte setzen das Politikschulbuch häufiger ein und greifen dabei vermehrt auf Arbeitsaufträge und Methodenvorschläge zurück. Lehrkräfte am Gymnasium nutzen es ebenfalls insgesamt häufiger. Befragte, die über sich selbst sagen, dass sie viel über die EU wissen, setzen die Schulbuchmaterialien eher ein. Lehrende, die von ihrer Selbstwirksamkeit im EU-Unterricht überzeugt sind, benötigen das Buch allerdings weniger für die generelle Planung und Vermittlung der Grundlagen als ihre weniger sicheren Kolleginnen und Kollegen. Lehrkräfte mit eher transmissiven lehr-lern-theoretischen Überzeugungen verwenden das Politikschulbuch häufiger als Leitmedium, während Lehrkräfte mit eher konstruktionsorientierten Überzeugungen es seltener für die Planung von Unterrichtseinheiten oder -stunden verwenden und das EUKapitel kritischer bewerten. Schülerinnen und Schüler nutzen das Politikschulbuch vor allem während des Unterrichts und zur Vorbereitung auf Klassenarbeiten. Lernende mit Migrationshintergrund greifen (auch unter Kontrolle ihres kulturellen Kapitals) signifikant häufiger zum Politikschulbuch, um den Unterricht vor- und nachzubereiten oder Fachbegriffe und Fremdwörter nachzuschlagen. Die Befragung zeigt zudem, dass Lehrkräfte sowie Schülerinnen und Schüler in der Bewertungsrangfolge einzelner Aspekte der Schulbücher weitgehend übereinstimmen. Während visuelle Inhalte und Korrektheit der Kapitel positiv bewertet werden, schneiden Methodenvorschläge, Schülerorientierung, Lebensweltbezug und Aktualität tendenziell schlechter ab.

\section{Literatur}

Baumert, Jürgen und Mareike Kunter. "Stichwort: Professionelle Kompetenz von Lehrkräften «, in: Zeitschrift für Erziehungswissenschaft 9 (4), 2006, 469-520.

Borries, Bodo von, Claudia Fischer, Sibylla Leutner-Ramm und Johannes MeyerHamme. Schulbuchverständnis, Richtlinienbezug und Reflexionsprozesse im Geschichtsunterricht. Eine qualitativ-quantitative Schüler- und Lehrerbefragung im Deutschsprachigen Bildungswesen 2002, Neuried: ars una, 2005. 
Dubberke, Thamar, Mareike Kunter, Nele McElvany, Martin Brunner und Jürgen Baumert. "Lerntheoretische Überzeugungen von Mathematiklehrkräften. Einflüsse auf die Unterrichtsgestaltung und den Lernerfolg von Schülerinnen und Schülern", in: Zeitschrift für pädagogische Psychologie 22, 2008, 193-206.

Flick, Uwe. Triangulation. Eine Einführung, 3. Aufl., Wiesbaden: VS Verlag für Sozialwissenschaften, 2011.

Hartinger, Andreas, Thilo Kleickmann und Birgit Hawelka. »Der Einfluss von Lehrervorstellungen zum Lehren und Lernen auf die Gestaltung des Unterrichts und auf motivationale Schülervariablen", in: Zeitschrift für Erziehungswissenschaft 9 (1), 2006, 110-126.

Oberle, Monika und Christian Tatje. »Schulbuchnutzung im Politikunterricht - eine empirische Studie«, in: Empirische Forschung zur schulischen Politischen Bildung, Sabine Manzel und Carla Schelle (Hg.), Wiesbaden: Springer VS, 2016, 113-125.

Oberle, Monika, Eva Weschenfelder und Georg Weißeno. »Beliefs als Element professioneller Kompetenz bei Politiklehrkräften in Deutschland «, in: Vorstellungen, Konzepte und Kompetenzen von Lehrpersonen der Politischen Bildung, Béatrice Ziegler (Hg.), Zürich: Rüegger Verlag, 2014, 124-137.

Rauch, Martin und Lothar Tomaschewski. Schulbücher für den Sachunterricht. Überblick - Analysen - Entscheidungshilfen. Frankfurt/M.: Arbeitskreis Grundschule e.V., 1986.

Tatje, Christian. Die Rolle des Schulbuchs bei der Vermittlung der Europäischen Integration. Nutzung und Wirkung im politischen Fachunterricht, Wiesbaden: Springer VS, 2017.

Weinbrenner, Peter. "Grundlagen und Methodenprobleme sozialwissenschaftlicher Schulbuchforschung", in: Schulbuchforschung, Richard Olechowski (Hg.), Frankfurt/M.: Peter Lang, 1995, 21-45. 


\section{Fazit und Ausblick}

Die Entscheidungen der Europäischen Union sind für den Alltag der Bürgerinnen und Bürger von großer Relevanz und zunehmend über europäische Beteiligungsverfahren wie die Europawahlen zu beeinflussen. Entsprechend ist die politische EU-Bildung ein wichtiger Bestandteil des Politikunterrichts. Die Komplexität und Dynamik der Europäischen Integration, die empfundene Bürgerferne sowie Fehlkonzepte und Vorurteile machen den Unterricht über die EU allerdings zu einer besonderen Herausforderung und stellen hohe Anforderungen an Schulbücher und Lehrende. Vor diesem Hintergrund ging das hier vorgestellte Forschungsprojekt »Vermittlung der Europäischen Integration im Schulbuch? Eine Studie zum politischen Fachunterricht" den Fragen nach, wie die Europäische Integration in deutschen Politikschulbüchern dargestellt wird und wie die Schulbücher von den Lehrenden und Lernenden genutzt und bewertet werden. Damit verband das Projekt auf innovative Weise gewinnbringend mehrere Ebenen der empirischen Schulbuchforschung.

Die hier vorgestellten Studienbefunde verdeutlichen, dass Schulbücher im alltäglichen Politikunterricht weiterhin ein relevantes Medium sind, um den von der KMK und den geltenden Kerncurricula ausgewiesenen Zielen der Europabildung nachzukommen. Insgesamt scheint ihre Nutzung deutlich höher zu sein, als die zunehmende Digitalisierung - und damit die dauerhafte digitale Verfügbarkeit von aktuellen Informationen - vermuten lässt.

Aus der umfassenden produktorientierten Analyse in Kapitel II lassen sich kategorienübergreifend einige Schlüsse ziehen, die für die künftige Entwicklung von Schulbuchmaterialien zur EU von Relevanz sind (vgl. auch 
Bischewski 2020). Positiv hervorzuheben ist, dass die Dynamik des Integrationsprozesses der EU in den meisten Schulbüchern gut aufgefangen wird. Politische Krisen und Transformationsprozesse - wie die Eurokrise oder der Vertrag von Lissabon - finden teilweise schon im selben Jahr Eingang in die Texte. Dennoch zeigt die qualitative Analyse auch, dass es dabei zu widersprüchlichen Darstellungen kommen kann, wenn die Offenheit des Integrationsprozesses in den Kapiteln nicht deutlich genug betont wird. Der rasche Wandel der politischen Wirklichkeit, der gerade im Falle der Europäischen Integration auch weitreichende institutionelle Veränderungen innerhalb weniger Jahre mit sich bringt, bleibt eine Herausforderung für die Vermittlung der EU im Politikschulbuch. Hinzu kommt, dass Schulbücher oft mehrere Jahre in der Schule verwendet werden - so waren in der 2015 durchgeführten Nutzungsstudie die aktuellsten verwendeten Schulbücher aus dem Jahr 2013. Es ist daher zu überlegen, wie sich Politikschulbücher zukünftig unter Rückgriff auf die Möglichkeiten der Digitalisierung weiterentwickeln können, um als Leitmedium des Politikunterrichts relevant bleiben und dem andauernden Problem der fehlenden Aktualität entgegentreten zu können.

Generell herrscht in den Büchern Einigkeit über die Vorteile der europäischen Einigung. Zwar werden teilweise auch kritische Einschätzungen über den aktuellen Zustand der EU sowie Vorurteile aufgegriffen, wobei Kritik an der europäischen Bürokratie im Vordergrund steht und unabhängig von Verlag und Schulform vorkommt. Dennoch muss konstatiert werden, dass viele Schulbücher eine krisen- und konfliktfreie Selbstverständlichkeit des europäischen Integrationsprozesses suggerieren und bestehende Kritik an der Europäischen Union zu wenig aufnehmen. Seit den Studien von Natterer (2001) und den Empfehlungen von Mellies, Migdalski und Kühberger (2007) sind hier - jedenfalls für die Schulbücher bis 2012/ 13 - kaum Fortschritte zu verzeichnen. Eine solche Darstellung wird angesichts der offenen Finalität des europäischen Integrationsprozesses, seiner regelmäßigen Krisen sowie der Zunahme euroskeptischer Stimmen in Parlamenten und Bevölkerung der politischen Wirklichkeit nicht gerecht. Didaktisch wird mit einem Verzicht auf die Thematisierung von vergangenen Krisen und Brüchen, wie der hier untersuchten Verfassungskrise, eine Chance vertan. Gerade in Zeiten, in denen (v. a. rechts-)populistische europafeindliche Stimmen mehr Zuspruch erhalten, könnte eine Ausein- 
andersetzung mit Zielen und Legitimationsansätzen der EU, ihren Problemen und kontrovers diskutierten Lösungsansätzen den Politikunterricht dabei unterstützen, Vorurteile und Fehlvorstellungen aufzubrechen und gleichzeitig dem Anspruch an Kontroversität gerecht zu werden. Den von KMK und Kerncurricula formulierten Zielen einer Stärkung des europäischen Bewusstseins und Förderung einer europäischen Identität kann durch das Eingehen auf die Errungenschaften der EU und die Verankerung der Europaorientierung im Grundgesetz weiterhin entsprochen werden.

Auch die von Weißeno (2004) kritisierte mangelhafte Darstellung des Mehrebenensystems bestätigt sich im deutschlandweiten Vergleich. Angesichts der Bedeutung der Fachsprache für die Ausbildung politischer Kompetenz ist auch der unzureichende Gebrauch von relevanten Fachbegriffen in den Schulbüchern kritisch zu bewerten. Hinsichtlich der politikdidaktischen Gestaltungsprinzipien Lebensweltbezug und Schülerorientierung sind die Schulbücher weiterhin verbesserungswürdig. Ein überraschendes, aber nicht minder kritisches Ergebnis der Analysen ist die Erkenntnis, dass zentrale Partizipationsmöglichkeiten auf europäischer Ebene in den Schulbüchern nur selten eingehend thematisiert werden. So wurde die Europawahl im Untersuchungszeitraum durchgehend wenig thematisiert und oftmals mit Verweis auf die geringe Wahlbeteiligung negativ besetzt. Andere Partizipationsmöglichkeiten auf europäischer Ebene, wie die mit dem Vertrag von Lissabon eingeführte Europäische Bürgerinitiative, kamen kaum vor. Gegen die weit verbreitete Unkenntnis hinsichtlich der Direktwahl zum Europäischen Parlament (vgl. Westle 2015) werden die bis 2013 veröffentlichten Schulbücher somit im Unterrichtseinsatz wenig ausrichten können. Allerdings zeigt sich hier eine positive Entwicklung, denn einige der 2016 und 2017 neu erschienenen Politikschulbücher (für Niedersachsen) beinhalten bereits einen eigenen Abschnitt zum Thema Bürgerpartizipation in der EU.

In Kapitel III konnten lediglich ausgewählte Ergebnisse der im Projekt durchgeführten Schüler- und Lehrerstudie zur Rolle des Schulbuchs im politischen Fachunterricht präsentiert werden. Eine deutliche Mehrheit der Lehrkräfte empfindet das Schulbuch für die Unterrichtsplanung als sehr wichtig oder eher wichtig. Das Politikschulbuch und seine EU-Kapitel werden von den Lehrerinnen und Lehrern dabei insbesondere zur Planung von ganzen Unterrichtseinheiten, aber auch von einzelnen Unterrichtsstunden sowie zur Vermittlung der Grundlagen, z.B. des Institutionenge- 
füges der EU, häufig verwendet. Das Schulbuch ist damit tatsächlich ein relevantes Leitmedium des Politikunterrichts. Zugleich zeigt sich, dass zumindest ein Teil der befragten Lehrkräfte im Unterricht zur EU erwartungsgemäß einen höheren Bedarf am Schulbuch als Leitmedium hat als im Politikunterricht generell. Fachfremd unterrichtende Lehrkräfte setzen das Schulbuch in ihrem Politikunterricht zur EU signifikant häufiger ein und greifen dabei auch vermehrt auf Arbeitsaufträge und Methodenvorschläge zurück als ihre fachlich ausgebildeten Kolleginnen und Kollegen. Auch Lehrkräfte mit eher transmissiven lehr-lern-theoretischen Überzeugungen nutzen das Politikschulbuch häufiger als Leitmedium, indem sie die EUEinheit mit dem Buch planen und es für die Erteilung von Hausaufgaben verwenden.

Das Nutzungsverhalten der Lehrkräfte unterscheidet sich außerdem nach Alter und Schulform. So zeigte sich, dass ältere Lehrkräfte das Schulbuch häufiger nutzen, um Hausaufgaben aufzugeben und Inhalte nacharbeiten zu lassen. Am Gymnasium kommt das Schulbuch bei der Unterrichtsplanung häufiger als an anderen Schulformen zum Einsatz und auch die darin angebotenen Materialien werden hier stärker genutzt. Tendenziell reflektieren die Lehrkräfte die Qualität des Buches und nutzen positiv beurteilte Bücher häufiger, wobei Personen mit eher konstruktionsorientierten lehrlern-theoretischen Überzeugungen den EU-Kapiteln kritischer gegenüberstehen. Schülerinnen und Schüler nutzen das Politikschulbuch vor allem während des Unterrichts und zur Vorbereitung auf Klassenarbeiten. Auffällig ist, dass Lernende mit Migrationshintergrund deutlich häufiger zum Politikschulbuch greifen, um den Unterricht vor- und nachzubereiten oder Fachbegriffe und Fremdwörter nachzuschlagen. Hierin liegt eine Chance der Politikschulbücher, mit geeigneten Angeboten zu Bildungsgerechtigkeit in der Migrationsgesellschaft beizutragen.

Etwa ein Viertel der befragten Lehrerinnen und Lehrer nutzt da Schulbuch zudem regelmäßig zur Auffrischung der eigenen Kenntnisse. Auch dies unterstreicht die bedeutende Rolle, die das Politikschulbuch heute für den Fachunterricht zur EU spielt, wobei die Nutzung des Buches als Informationsquelle der Lehrkräfte angesichts der eingeschränkten Aktualität (gerade von nicht frisch zugelassenen Schulbüchern), der mangelnden Kontroversität und der erforderlichen Komplexitätsreduktion durchaus auch kritisch zu sehen ist. Dies weist auf den Bedarf an Lehrerfortbildungen 
zur Vermittlung der EU mit fachwissenschaftlichen und fachdidaktischen Anteilen hin (vgl. Oberle und Forstmann 2015; Schöne und Immerfall 2015). Zugleich unterstreichen die vorliegenden Befunde zur intensiven und vielfältigen Nutzung der Bücher durch Lehrende und Lernende, dass sich Bemühungen um eine Qualitätssteigerung der Schulbuchkapitel für den Politikunterricht in mehrfacher Hinsicht lohnen.

Die Mehrheit der befragten Lehrkräfte bewertet die Darstellung der Geschichte und der Grundlagen der EU, die Kohärenz verschiedener Texte, die Grafiken und Aufgabenstellungen sowie den Aufbau der EU-Kapitel als gut oder sehr gut. Besonders Karikaturen, Schaubilder oder Grafiken werden gern verwendet, während Autorentexte oder Methodenvorschläge weniger Anklang finden. Deutliche Kritik üben die Lehrkräfte allerdings an der mangelnden Aktualität der verwendeten EU-Kapitel und dem Mangel an Schülerorientierung und Lebensweltbezug. In der Rangfolge der Bewertung unterschiedlicher Schulbücher stimmen die Lernenden mit den Lehrkräften weitgehend überein, wobei Lehrkräfte die Bücher differenzierter bewerten und größere Qualitätsunterschiede ausmachen. Insgesamt bewerten die Schülerinnen und Schüler die EU-Kapitel durchaus positiv, wobei sie mit dem EU-Teil zufriedener sind als mit dem jeweiligen Schulbuch insgesamt. Die EU-Kapitel werden von ihnen überwiegend als verständlich, wenig kompliziert und übersichtlich wahrgenommen. Der Mehrheit der Schülerinnen und Schüler fehlt es dabei allerdings wie den Lehrkräften an Lebensweltbezug und die Aufgabenstellungen in den Büchern finden sie eher langweilig. Fotos von Jugendlichen und Abbildungen von Schülermeinungen zur EU-Politik, die in den untersuchten Büchern eher selten vorkommen, werden von einer knappen Mehrheit der Befragten positiv bewertet und verstärkt gewünscht.

Vertiefende Analysen und latent berechnete Modelle zur Rolle des Schulbuchs im Politikunterricht sowie der Beurteilung insbesondere der EU-bezogenen Kapitel aus Schüler- und Lehrerperspektive finden sich bei Tatje (2017) sowie Oberle und Tatje (2016). Die hier vorgestellten Ergebnisse unterstreichen jedoch bereits, dass Schulbücher noch immer eine bedeutende Rolle für den Politikunterricht spielen. Gefordert ist daher eine größere Beachtung dieses Mediums seitens der politikdidaktischen Forschung, und zwar sowohl mittels wirkungsorientierter Studien als auch durch kritische produktorientierte und schließlich auch prozessorientierte Analysen, die auch den Produktionsprozess der Bücher in den Blick nehmen. Dabei 
sollten auch die Potenziale des Schulbuchs für die politische Bildung in der Migrationsgesellschaft eine besondere Beachtung erfahren.

\section{Literatur}

Bischewski, Marret. Von Laeken bis Lissabon: Der Reformprozess der Europäischen Union in deutschen Politikschulbüchern. Eine computergestützte Inhaltsanalyse, Dissertation, Göttingen: Georg-August-Universität Göttingen, 2020.

Mellies, Dirk, Pawel Migdalski, Christoph Kühberger u. a. Empfehlungen zum Umgang mit Geschichte anhand von Schulbuchkapiteln zur Geschichte der Europäischen Union, 2007, http://www.demokratiezentrum.org/fileadmin/media/pdf/ku ehberger_eu.pdf, zuletzt geprüft am 05. September 2020.

Natterer, Alexandra. Europa im Schulbuch. Die Darstellung der europäischen Einigung in baden-württembergischen Schulbüchern für Geschichte und Gemeinschaftskunde der Sekundarstufe I, Grevenbroich: Omnia, 2001.

Oberle, Monika und Johanna Forstmann. "Lehrerfortbildungen zur politischen EUBildung - eine empirische Begleitstudie«, in: Die Europäische Union erfolgreich vermitteln. Perspektiven der politischen EU-Bildung heute, Monika Oberle (Hg.), Wiesbaden: Springer VS, 2015, 193-209.

Oberle, Monika und Christian Tatje. »Schulbuchnutzung im Politikunterricht - eine empirische Studie«, in: Empirische Forschung zur schulischen Politischen Bildung, Sabine Manzel und Carla Schelle (Hg.), Wiesbaden: Springer VS, 2016, 113-125.

Schöne, Helmar und Stefan Immerfall. »EU-Bildung in der Schule - Erfahrungen und Desiderate", in: Die Europäische Union erfolgreich vermitteln. Perspektiven der politischen EU-Bildung heute, Monika Oberle (Hg.), Wiesbaden: Springer VS, 2015, 67-80.

Tatje, Christian. Die Rolle des Schulbuchs bei der Vermittlung der Europäischen Integration. Nutzung und Wirkung im politischen Fachunterricht, Wiesbaden: Springer VS, 2017.

Weißeno, Georg. »Konturen einer europazentrierten Politikdidaktik - Europäische Zusammenhänge verstehen lernen «, in: Europa verstehen lernen. Eine Aufgabe des Politikunterrichts, ders. (Hg.), Bonn: Bundeszentrale für Politische Bildung, 2004, 108-125.

Westle, Bettina. "Wissen um die Direktwahl des Europäischen Parlaments - eine Frage des Alters? Deutsche im westeuropäischen Vergleich", in: Die Europäische Union erfolgreich vermitteln. Perspektiven der politischen EU-Bildung heute, Monika Oberle (Hg.), Wiesbaden: Springer VS, 2015, 39-63. 


\section{Empfehlungen für die Darstellung der Europäischen Union in Politikschulbüchern}

Die vorliegende Studie hat aufgezeigt, dass Lehrkräfte das Politikschulbuch häufig zur Planung von Unterrichtseinheiten und einzelnen Unterrichtsstunden und damit als Leitmedium ihres EU-Unterrichts verwenden. Aufgrund der nach wie vor großen Relevanz des Politikschulbuchs sollte dieses klassische Unterrichtsmedium seitens der politikdidaktischen Forschung und der Schulbuchforschung größere Beachtung erfahren. Eine Zusammenarbeit mit den Produzierenden von Schulbüchern, beispielsweise in Projekten mit wissenschaftlicher Begleitforschung, könnte dabei gewinnbringend sein.

Das Schulbuch ist nicht zuletzt aufgrund der einfachen Zugänglickeit für alle ein wichtiges didaktisches Hilfsmittel im Unterricht. Die folgenden Empfehlungen basieren auf den Ergebnissen beider Teilstudien des hier vorgestellten Forschungsprojekts und sind Vorschläge dazu, wie das "Leitmedium zum Anfassen « sinnvoll ergänzt und die Darstellung der EU in den Schulbüchern verbessert werden können.

Zentrale Aspekte mit Verbesserungsbedarf

1) Aktualität und Dynamik - Darstellung der Offenheit und Dynamik des Integrationsprozesses

2) Multiperspektivität und Kontroversität - Multiperspektivische Darstellung der kontroversen Auseinandersetzungen um politische Entscheidungen und Erwähnung von Krisen und Problemen der europäischen Einigung 
3) Terminologie - Einführung und kohärente Verwendung von Fachbegriffen

4) Exemplarität - Erläuterung politischer Kompetenzen, Prozesse und Entscheidungen anhand von Beispielen

5) Schülerorientierung und Lebensweltbezug - Veranschaulichung des Bezugs von Entscheidungsprozessen der EU zu Lebenswelt und Alltag der Schülerinnen und Schüler

6) Bürgerleitbilder und Partizipation - Darstellung der EU-bezogenen Partizipationsmöglichkeiten sowie der Anknüpfungspunkte für das Engagement junger Bürgerinnen und Bürger

7) Flexible Darstellung - Schaffung von Verwendungsmöglichkeiten für verschiedene Nutzungstypen

\section{Zu 1) Aktualität und Dynamik}

Schulbücher nehmen politische Entwicklungen (z. B. Vertragsänderungen) in einem Zeitraum von maximal zwei Jahren auf. Allerdings führen Krisen, wie die Debatte um die Verfassungsreform, zugleich zu Problemen in der Darstellung und auch zu widersprüchlichen Aussagen innerhalb eines Kapitels. Einige der untersuchten Bücher behandeln z. B. die Verfassungsdebatte als einen offenen Prozess, obgleich der Ratifizierungsprozess des Europäischen Verfassungsvertrags zum Zeitpunkt der Veröffentlichung bereits gescheitert war. Dadurch entstehen teils irreführende Darstellungen, in denen behauptet wird, die Verfassung gäbe es doch, sie hieße jetzt jedoch »Reformvertrag«. Seitens der Lehrenden wird die Aktualität der Schulbücher als eher schlecht bewertet.

- Es ist notwendig, die Offenheit und Dynamik des europäischen Integrationsprozesses zu betonen und die im Schulbuch enthaltenen Informationen als zeitgebunden darzustellen. Dadurch können auch Frustrationserfahrungen angesichts eines schnell veralteten EU-Wissens vermieden werden.

- Auch bei Aktualisierungen von Schulbüchern sollte auf Kohärenz der Darstellungen geachtet werden.

- Eine Lösung könnten zunehmende Online-Begleitung (auch durch ergänzende Linklisten zu weiterführenden Informationen z. B. der EU oder 
der Bundeszentrale für politische Bildung), unbürokratische Lehrerhandreichungen und Aktualisierungen der Schulbücher über einen WebZugriff sein. Methodenseiten sollten einen Rahmen und didaktische Ansätze anbieten, in Bezug auf das Thema jedoch inhaltlich flexibel bleiben.

Zu 2) Multiperspektivität und Kontroversität

Der Integrationsprozess wird in den Schulbüchern überwiegend als geradlinig dargestellt - oftmals visuell unterstützt durch die Abbildung der historischen Zeitleiste als »Wanderung« oder »Radtour«. Problematische Entwicklungen und Legitimationskrisen (»Politik des leeren Stuhls«, Maastricht-Krise, Verfassungsdebatte) kommen in den aktuelleren Büchern kaum vor. Die Vertiefung der politischen Integration wird nicht in Frage gestellt und EU-Skeptizismus (wenn überhaupt) nicht differenziert dargestellt. Gründe, warum Bürgerinnen und Bürger eine bestimmte Reform oder politische Entscheidung der EU ablehnen, werden nicht genannt. Auch aus der Darstellung des Gesetzgebungsprozesses gehen die Konflikthaftigkeit und die unterschiedlichen Positionen der beteiligten Akteure selten hervor. Interessenvertreter, Nichtregierungsorganisationen und Lobbyisten bleiben bislang oftmals ausgeblendet. In den Darstellungen wird dabei zwar von Zustimmung oder Ablehnung eines Vorschlags gesprochen, aber selten auf Argumente eingegangen, die für oder gegen die Annahme sprechen. Der politische Prozess als kontroverse Debatte, als Ringen um gemeinsame Lösungen und Mehrheiten vor dem Hintergrund unterschiedlicher Interessen, wird so nicht veranschaulicht.

- Die verschiedenen Positionen zur weiteren Integration sollten deutlich und die Vielzahl an Akteuren und Meinungen zum Integrationsprozess transparenter gemacht werden. Sowohl politikwissenschaftliche als auch politisch-gesellschaftliche Ansichten zu den verschiedenen Integrationsmodellen können (in schülergerechter Sprache) vorgestellt werden.

- Auch bei politischen Entscheidungen in konkreten Politikfeldern sollte auf die Darstellung von Interessenvielfalt und Kontroversität geachtet werden. Das könnte mit der stärkeren Hervorhebung der Relevanz der 
Europawahlen für den Ausgang politischer Entscheidungen der EU verbunden werden (siehe Punkt 6, Bürgerleitbilder und Partizipation).

- Die Zustimmung der Gesellschaft zur EU ist nicht ungeteilt (siehe Brexit) und belastet die Europäische Identität. Zu beobachten ist, dass sich der permissive consensus, welcher die Europäische Integration jahrzehntelang getragen hat, teilweise zu einem constraining dissensus entwickelt hat. Daher sollten die Schulbücher auch EU-kritische Meinungen aufnehmen, die im Unterricht gemeinsam mit der Lehrkraft aufgearbeitet und diskutiert werden können.

- Die Schulbücher sollten nicht nur für Europa werben, sondern auf strukturelle Probleme der EU hinweisen. Es gilt, nicht nur das Faktische, sondern auch das Offene und Kontroverse im Politikprozess zu verdeutlichen - sowohl auf Ebene der polity (Form von Politik, politisches System der EU), als auch auf Ebene der policies (Inhalte von Politik, politische Entscheidungen der EU).

\section{Zu 3) Terminologie}

Definitionen zu bestimmten Begriffen oder Verweise auf ein Glossar gibt es nur in einzelnen Büchern. In kaum einem Buch wird überhaupt der Begriff "Europäische Union« eingeführt bzw. die Abkürzung EU definiert, stattdessen werden viele Fachbegriffe vorausgesetzt. Problematisch sind insbesondere die Unterscheidung Ministerrat/Rat der EU und Europäischer Rat und die jeweilige »Ratspräsidentschaft«. Hier herrscht oft Unklarheit in den Büchern. Weitere Beispiele sind die »Europäische Bürgerinitiative« und die "Direktwahl des Europäischen Parlaments«: diese Konzepte werden meist nur umschrieben oder indirekt benannt - daher ist es unwahrscheinlich, dass die Schülerinnen und Schüler später eigenständig darauf zurückgreifen können.

- Die Schulbücher sollten Fachbegriffe nicht vermeiden, sondern sie angemessen nutzen, um der Relevanz der Fachsprache für die Entwicklung politischer Kompetenzen gerecht zu werden. Fachbegriffe sollten dabei gezielt eingeführt, verständlich erläutert und einheitlich verwendet werden. 
- Um insbesondere auf die Schülerinnen und Schüler einzugehen, die das Schulbuch vorwiegend zum Nachschlagen oder Vorbereiten der nächsten Unterrichtsstunde benutzen (unserer Studie zufolge gerade auch Schülerinnen und Schüler mit Migrationshintergrund), sollten Fachbegriffe als solche gekennzeichnet und, beispielsweise in einem Glossar, in für die Zielgruppe verständlicher Sprache definiert werden. Dabei sollte eine Differenzierung nach Anforderungsbereichen und sprachlichen Fähigkeiten erfolgen.

\section{Zu 4) Schülerorientierung und Lebensweltbezug}

Die Schülerorientierung wird in den Büchern eher schlechter bewertet. Die gewählten Beispiele zur Veranschaulichung des Politikprozesses sind selten schülerorientiert, da sie die Lebenswelt der Schülerinnen und Schüler nicht - oder nur oberflächlich - miteinbeziehen. Die Texte verdeutlichen zwar überwiegend den großen Einfluss der EU auf den Alltag der Bürgerinnen und Bürger, dabei wird jedoch selten mit konkreten Beispielen aus der Lebenswelt der Lernenden (z. B. der eigenen Region bzw. jugendrelevanten Themen) gearbeitet. Auch visuell wird die verbreitete Wahrnehmung einer Bürgerferne der EU in den Schulbüchern unterstützt, denn bei der Darstellung überwiegen (weiterhin) Impressionen von Gebäuden der EU, diese meist in Außenansicht. Einblicke z. B. in einen Plenarsaal werden meist nur aus einer entfernten Perspektive und (sofern das Plenum gefüllt ist) mit Blick auf die Rücken der Politikerinnen und Politiker geboten. Generell werden Politikerinnen und Politiker eher in Gruppen dargestellt, Einzelporträts und Interviews mit bestimmten Politikerinnen und Politikern sind in den EU-Kapiteln selten enthalten, was zum Eindruck der Distanz zur Europäischen Union beiträgt.

- Fotos von Jugendlichen und die Abbildung von Schülermeinungen zur EU finden sich nicht in allen Büchern, werden aber in der Befragung der Schülerinnen und Schüler eher positiv bewertet. Sofern diese Fotos und Sprechblasen nicht rein illustrativen Charakter haben, sondern zur Diskussion anregen, können sie der Schülerorientierung zuträglich sein.

- Lebensnahe Themen wie Jugendpolitik und Bildung kommen in den Kapiteln zur EU bislang kaum vor, könnten aber durchaus als Beispiel für 
die verschiedenen Zuständigkeitsbereiche der EU und der Mitgliedsstaaten herangezogen werden.

- Trends und Interessen bei Jugendlichen ändern sich rasant, daher ist es sicherlich anspruchsvoll, geeignete Themen und Inhalte für Schulbücher zu finden. Dennoch könnte zumindest die visuelle Distanz zu Brüssel durch Verwendung neuer und anderer Bilder gemildert werden. Außerdem könnten die Aufgabenstellungen und Texte angepasst und durch Online-Verweise ergänzt werden (z. B. Links zu virtuellen Touren durch die Gebäude der EU).

\section{Zu 5) Exemplarität}

Generell fällt auf, dass das lernförderliche Potenzial der Exemplarität in den Büchern zu wenig genutzt wird. Beispiele für Kompetenzbereiche, politische Entscheidungsprozesse und Errungenschaften der Europäischen Union kommen nicht häufig genug vor. Gesetzgebungsverfahren werden oftmals mit den üblichen Pfeildiagrammen dargestellt - reines Schaubildwissen trägt jedoch nur begrenzt zu einem Verständnis politischer Prozesse bei.

- Sowohl für Zuständigkeitsbereiche der EU und ihrer einzelnen Institutionen als auch für politische Prozesse und politische Entscheidungen sollten zur Veranschaulichung häufiger Beispiele genutzt werden.

- Anhand des Politikzyklus können involvierte Akteure und kontroverse Interessen sowie die Offenheit und grundsätzliche Unabgeschlossenheit politischer Entscheidungsprozesse exemplarisch verdeutlicht werden. Auch das ordentliche Gesetzgebungsverfahren der EU sollte nicht nur als abstraktes Diagramm, sondern zusätzlich mit Hilfe eines konkreten Beispiels erläutert werden.

- Gerade auch regionale Beispiele für die Bedeutung der EU sollten in den Schulbüchern verstärkt vorkommen. 


\section{Zu 6) Bürgerleitbilder und Partizipation}

Ein Leitziel der politischen Bildung ist es, Jugendliche zu politischer Teilhabe zu befähigen. Allerdings werden die Möglichkeiten zur politischen Partizipation auf europäischer Ebene in den Schulbüchern nur am Rande behandelt. Die Darstellung des Themas Europawahl als zentrale konventionelle Partizipationsmöglichkeit wird auch von den Lehrkräften entsprechend negativ beurteilt, wenngleich sie das Thema für sehr relevant halten. In den meisten Texten wird dadurch indirekt einer Output-Legitimierung der EU mehr Bedeutung beigemessen als der Input-Legitimierung. Die Europawahl kommt zwar mittlerweile in fast allen aktuellen Schulbüchern vor, aber oftmals wird sie nur erwähnt und das Wahlrecht wird nicht ausreichend erklärt; und wenn mehr berichtet wird, ist das Thema aufgrund der geringen Wahlbeteiligung negativ besetzt. Proteste/Demonstrationen und Aktionen der Zivilgesellschaft werden lediglich zur Illustration in Form von Fotos eingebracht. Ein Bezug im Text ist selten zu finden, abermals wird auf das Aufzählen von Argumenten verzichtet.

- Die Wahlen zum Europäischen Parlament als die zentrale Möglichkeit der Mitbestimmung der Bürgerinnen und Bürger an den politischen Entscheidungen der EU sollten in den Schulbüchern schwerpunktmäßig behandelt werden. Dabei sollte auf das Wahlrecht und seine Besonderheiten, die Bedeutung des Wahlausgangs für europäische Entscheidungsprozesse sowie auf Wahlprogramme von Parteien und/oder exemplarisch auf Kandidatinnen und Kandidaten und deren politische Ziele eingegangen werden.

- Damit in Zusammenhang steht das Erfordernis, die (bedeutsame) Rolle des Europäischen Parlaments im ordentlichen Gesetzgebungsfahren zu erläutern, exemplarisch darzustellen und auch Einblicke in seine Arbeit in länderübergreifenden Fraktionen und Ausschüssen zu geben. Ein exemplarischer Arbeitstag einer Abgeordneten oder eines Abgeordneten kann deren Tätigkeit veranschaulichen. Auch Kritik an fehlenden Befugnissen des Parlaments, wie das nicht vorhandene legislative Initiativrecht, sollte Erwähnung finden (siehe Punkt 2, Kontroversität).

- Neben den Europawahlen sollten auch weitere Partizipationsformen wie die mit dem Vertrag von Lissabon eingeführte Europäische Bürgerini- 
tiative, (Online-)Petitionen, Demonstrationen und die Kontaktaufnahme mit Abgeordneten und Ombudspersonen vorgestellt werden.

- Ausgehend von dem Leitbild der politischen Bildung und im Sinne der Schülerorientierung sollte es einen Überblick über die zentralen Partizipationsmöglichkeiten geben, denn Information und Wissen (Stichwort: Nutzung des Schulbuchs zum Nachschlagen) können Partizipation fördern.

\section{Zu 7) Flexible Darstellung}

Lehrerinnen und Lehrer, die Politik (oder Inhaltsähnliches) studiert haben, sind deutlich unwahrscheinlicher dem Typ häufiger Schulbuchnutzung zuzuordnen und verwenden das EU-Kapitel seltener zur Planung ihres Unterrichts sowie für die Vergabe von Hausaufgaben. Lehrkräfte mit eher transmissiven lehr-lern-theoretischen Überzeugungen nutzen das Politikschulbuch häufiger als Leitmedium, während Lehrkräfte mit konstruktionsorientierten Überzeugungen es seltener für die Planung von Unterrichtseinheiten oder -stunden nutzen und kritischer bewerten.

- Bücher und Handreichungen sollten besonders fachfremde Lehrkräfte berücksichtigen und auch grundlegende fachwissenschaftliche sowie fachdidaktische Hinweise und Informationen liefern. So kann das Schulbuch auch unerfahrenen Lehrenden als Orientierung und Unterstützung für ihren Unterricht dienen.

- Offenbar mangelt es an geeigneten Aufgabenstellungen und Methodenvorschlägen im Sinne einer eher konstruktivistisch geprägten LehrLern-Theorie. Dies sollte verbessert werden, um die Nutzungsmöglichkeiten des Schulbuchs für einen Unterricht, der auf Eigenaktivität und kognitive Aktivierung der Lernenden setzt, zu erhöhen. 


\section{Literaturangaben zu den untersuchten Schulbüchern (DEUS und NIS)}

Deutschlandweites Gesamtsample DEUS $(n=87)$; Niedersächsisches Teilsample NIS $(\mathrm{n}=30)$, durch Fettdruck hervorgehoben Abkürzungen bei den Schulformen wie folgt: Gesamtschule (GS), Gymnasium (GY), Hauptschule (HS), Realschule (RS)

\begin{tabular}{|l|l|l|}
\hline Titel & $\begin{array}{l}\text { Bundes- } \\
\text { land }\end{array}$ & $\begin{array}{l}\text { Schul- } \\
\text { form }\end{array}$ \\
\hline $\begin{array}{l}\text { Adam, Annette, Hans-Rudi Bratschke, Michael Gollert, } \\
\text { Cathrin Schreier und Hermann Tallen. Sozialkunde, Klassen 9/ } \\
\text { 10, Mecklenburg-Vorpommern, Hannover: Schroedel, 2003. }\end{array}$ & MV & $\begin{array}{l}\text { HS, GS, } \\
\text { RS }\end{array}$ \\
\hline $\begin{array}{l}\text { Adam, Annette, Cathrin Schreier und Heinz-Ulrich Wolf. } \\
\text { Demokratie heute 9/10, Sozialkunde Thüringen, } \\
\text { Braunschweig: Schroedel, 2007. }\end{array}$ & TH & $\begin{array}{l}\text { HS, GS, } \\
\text { RS }\end{array}$ \\
\hline $\begin{array}{l}\text { Adam, Annette, Cathrin Schreier und Heinz-Ulrich Wolf. } \\
\text { Demokratie heute 9/10, Sozialkunde Sachsen-Anhalt, } \\
\text { Braunschweig: Schroedel, 2007. }\end{array}$ & ST & HS, GS \\
\hline $\begin{array}{l}\text { Alber, Jürgen, Birgit Neuer, Klaus Witzigmann und Heinz- } \\
\text { Ulrich Wolf. Trio 4. Welt/Zeit/Gesellschaft, Klasse 10, } \\
\text { Werkrealschule Baden-Württemberg, Braunschweig: } \\
\text { Schroedel, 2012. }\end{array}$ & BW & $\begin{array}{l}\text { HS, GS, } \\
\text { RS }\end{array}$ \\
\hline $\begin{array}{l}\text { Andreas, Heinz (Hg.). Politik Real, Gesamtband für die Real- } \\
\text { und Regionalschule in Rheinland-Pfalz, Troisdorf: } \\
\text { Bildungsverlag Eins, 2006. }\end{array}$ & RP, SL & $\begin{array}{l}\text { HS, GS, } \\
\text { RS }\end{array}$ \\
\hline
\end{tabular}


(Fortsetzung)

\begin{tabular}{|c|c|c|}
\hline Titel & $\begin{array}{l}\text { Bundes- } \\
\text { land }\end{array}$ & $\begin{array}{l}\text { Schul- } \\
\text { form }\end{array}$ \\
\hline $\begin{array}{l}\text { Augustin, Christian, Guiskard Eck, Hans-Ullrich } \\
\text { Kötteritzsch, Antje Krawietz-Hüll, Anna Kreutz, Krystyna } \\
\text { Kusserow, Harald-Matthias Neumann, Ilona Olesch, Georg } \\
\text { Pinter und Martin Spätling. Das IGL-Buch 3, Unterrichtswerk } \\
\text { für Gesellschaftslehre und Weltkunde, Stuttgart: Klett, 2011. }\end{array}$ & NI & GY, GS \\
\hline $\begin{array}{l}\text { Bauer, Thomas, Walther Grosser, Evelyn Kuchler, Rudolf } \\
\text { Kunz, Renata Libera, Astrid Senft und Herbert Wagner. Trio 8, } \\
\text { Geschichte, Sozialkunde, Erdkunde, Hauptschule Bayern, } \\
\text { Hannover: Schroedel, 2005. }\end{array}$ & BY & HS, GS \\
\hline $\begin{array}{l}\text { Bauer, Thomas, Walther Grosser, Evelyn Kuchler, Rudolf } \\
\text { Kunz, Renata Libera, Astrid Senft und Herbert Wagner. Trio 9, } \\
\text { Geschichte, Sozialkunde, Erdkunde, Hauptschule Bayern, } \\
\text { Braunschweig: Schroedel, } 2007 .\end{array}$ & BY & HS, GS \\
\hline $\begin{array}{l}\text { Bax, Michael, Kornelia Bähre, Michael Gollert, Harald } \\
\text { Mertins, Klaus Rieckmann, Bernd-Wolfgang Vahldiek und } \\
\text { Dieter Vorrath. Trio 9/10, Geschichtlich-soziale Weltkunde, } \\
\text { Neubearbeitung, Hannover: Schroedel, 2003. }\end{array}$ & NI & GY, GS \\
\hline $\begin{array}{l}\text { Beckmann-Schulz, Petra, Joachim Detjen, Werner Launhardt, } \\
\text { Karl-Heinz Meyer, Albrecht Pohle, Horst Sprenger, Hermann } \\
\text { Tallen und Friedrich-Wilhelm Wedemeyer. Mensch und } \\
\text { Politik SI, Braunschweig: Schroedel, 2003. }\end{array}$ & NI & GY, GS \\
\hline $\begin{array}{l}\text { Berger-von der Heide, Thomas. Entdecken und Verstehen } 3 . \\
\text { Vom Ende des Ersten Weltkriegs bis zur Gegenwart, } \\
\text { Geschichtsbuch für Realschulen in Niedersachsen, Berlin: } \\
\text { Cornelsen, 2006. }\end{array}$ & NI & RS \\
\hline $\begin{array}{l}\text { Berger-von der Heide, Thomas (Hg.). Politik entdecken, Bd. 2, } \\
\text { Berlin: Cornelsen, } 2009 .\end{array}$ & NRW & RS \\
\hline $\begin{array}{l}\text { Berger-von der Heide, Thomas (Hg.). Entdecken und } \\
\text { Verstehen 3, Arbeitsbuch für Gesellschaftslehre/Weltkunde, } \\
\text { Berlin: Cornelsen, } 2010 .\end{array}$ & NI & $\begin{array}{l}\text { HS, GS, } \\
\text { RS }\end{array}$ \\
\hline $\begin{array}{l}\text { Berger-von der Heide, Thomas und Tanja Laspe. Politik } \\
\text { entdecken, 9/10, Ausgabe N, Berlin: Cornelsen, } 2009 .\end{array}$ & NI & RS \\
\hline
\end{tabular}




\section{(Fortsetzung)}

\begin{tabular}{|c|c|c|}
\hline Titel & $\begin{array}{l}\text { Bundes- } \\
\text { land }\end{array}$ & $\begin{array}{l}\text { Schul- } \\
\text { form }\end{array}$ \\
\hline $\begin{array}{l}\text { Bernhardt, Markus, Johannes Derichs, Katja Hoffmann, } \\
\text { Siegfried Hoffmann, Monika Lenniger, Wolfgang Pankratz, } \\
\text { Torsten Steinberg, Hildegard Wacker, Monika Wendorf und } \\
\text { Gerald Wieziolkowski. Durchblick. Geschichte/Politik/ } \\
\text { Erdkunde 9/10, Niedersachsen, Braunschweig: Westermann, } \\
\text { 2007. }\end{array}$ & NI & HS, GS \\
\hline $\begin{array}{l}\text { Betz, Christine, Sabine Hoffmann, Thomas Volkert und } \\
\text { Friedrich Wölfl. Politik aktuell 9. Zusammenleben im } \\
\text { 21. Jahrhundert, Unterrichtswerk für das Wirtschafts- und } \\
\text { Sozialwissenschaftliche Gymnasium in Bayern, Bamberg: C.C. } \\
\text { Buchner, 2011. }\end{array}$ & BY & GY \\
\hline $\begin{array}{l}\text { Brandes, Heinz-Werner und Hansjörg Kaiser (Hg.). Team } \\
E W G \text { 3, Erdkunde, Wirtschaftskunde, Gemeinschaftskunde, } \\
\text { Paderborn: Schöningh, } 2006 .\end{array}$ & BW, SL & RS \\
\hline $\begin{array}{l}\text { Brandt, Uwe und Angela Hitzschke. Mensch und Politik SI, } \\
\text { Bd. 2, Sachsen, Braunschweig: Schroedel, } 2006 .\end{array}$ & $\mathrm{SN}$ & GY \\
\hline $\begin{array}{l}\text { Bröckel, Thorsten (Hg.). Menschen Zeiten Räume. Welt Zeit } \\
\text { Gesellschaft 4, Arbeitsbuch für den Fächerverbund Welt-Zeit- } \\
\text { Gesellschaft an Hauptschulen in Baden-Württemberg, Berlin: } \\
\text { Cornelsen, 2007. }\end{array}$ & $\mathrm{BW}, \mathrm{SL}$ & $\begin{array}{l}\text { HS, GS, } \\
\text { RS }\end{array}$ \\
\hline $\begin{array}{l}\text { Brockhausen, Alfons, Dieter Deiseroth, Jelko Peters, Hans- } \\
\text { Jürgen Smula, Gregor Wegmann und Heinz-Ulrich Wolf. } \\
\text { Demokratie heute 3, Politik, Nordrhein-Westfalen, } \\
\text { Braunschweig: Schroedel, 2011. }\end{array}$ & NRW & RS \\
\hline $\begin{array}{l}\text { Brockhausen, Alfons, Jelko Peters, Hans-Jürgen Smula und } \\
\text { Heinz-Ulrich Wolf (Hg.). Demokratie heute 9/10, Politik, } \\
\text { Nordrhein-Westfalen, Braunschweig: Schroedel, 2007. }\end{array}$ & NRW & RS \\
\hline $\begin{array}{l}\text { Brokemper, Peter, Elisabeth Köster und Dieter Potente (Hg.). } \\
\text { Entdecken und Verstehen 3, Arbeitsbuch für Geschichte/ } \\
\text { Politik an Hauptschulen in Nordrhein-Westfalen, Berlin: } \\
\text { Cornelsen, 2007. }\end{array}$ & NRW & HS, GS \\
\hline $\begin{array}{l}\text { Castner, Jan, Dörthe Hecht, Ulla Oppenländer und Petra } \\
\text { Reiter-Mayer. Politik.21, Politik und Wirtschaft, Bd. 3, } \\
\text { Bamberg: C.C. Buchner, 2012. }\end{array}$ & $\mathrm{HE}$ & $\begin{array}{l}\text { HS, GS, } \\
\text { RS }\end{array}$ \\
\hline $\begin{array}{l}\text { Castner, Jan, Jan Weber, Ulla Oppenländer, Petra Reiter- } \\
\text { Mayer und Hartwig Riedel. Politik.21, Niedersachsen, Bd. 2, } \\
\text { Bamberg: C.C. Buchner, 2012. }\end{array}$ & NI & RS \\
\hline
\end{tabular}




\section{(Fortsetzung)}

\begin{tabular}{|c|c|c|}
\hline Titel & $\begin{array}{l}\text { Bundes- } \\
\text { land }\end{array}$ & $\begin{array}{l}\text { Schul- } \\
\text { form }\end{array}$ \\
\hline $\begin{array}{l}\text { Cesar-Rothfeld, Brigitte, Frank Eichhorn, Detlef Eichner, Kai } \\
\text { Jäckel, Karl-Heinz Kolb, Harald Mertins und Dieter Vorrath. } \\
\text { Trio 9/10, Geschichte, Erdkunde, Politik, Braunschweig: } \\
\text { Schroedel, 2010. }\end{array}$ & NI & $\begin{array}{l}\text { HS, GS, } \\
\text { RS }\end{array}$ \\
\hline $\begin{array}{l}\text { Christoffer, Sven, Michaela Dominik, Guiskard Eck, Eckhard } \\
\text { Hanke, Helmut Heimbach, Uli Jungbluth, Klaus Leinen, } \\
\text { Harald-Matthias Neumann und Antonius Wollschläger. } \\
\text { Mitmischen Plus 3, Stuttgart/Leipzig: Klett, } 2010 .\end{array}$ & $\mathrm{RP}, \mathrm{SL}$ & $\begin{array}{l}\text { HS, GS, } \\
\text { RS }\end{array}$ \\
\hline $\begin{array}{l}\text { Deichmann, Carl (Hg.). Politisch denken. Politisch handeln, } \\
\text { Gemeinschaftskunde/Rechtserziehung, Klassen 9/10, } \\
\text { Landesausgabe Sachsen, Leipzig: Militzke, } 2005 .\end{array}$ & SN & $\mathrm{HS}, \mathrm{GS}$ \\
\hline $\begin{array}{l}\text { Deichmann, Carl (Hg.). Politisch denken. Politisch handeln, } \\
\text { Sozialkunde, Klassen 9/10, Landesausgabe Thüringen } \\
\text { Gymnasium, Leipzig: Militzke, } 2006 .\end{array}$ & $\mathrm{TH}$ & GY \\
\hline $\begin{array}{l}\text { Deiseroth, Dieter, Marc A. Gollon und Heinz-Ulrich Wolf. } \\
\text { Demokratie heute, Sozialkunde, Rheinland-Pfalz/Saarland, } \\
\text { Braunschweig: Schroedel, } 2009 .\end{array}$ & $\mathrm{RP}, \mathrm{SL}$ & GY, GS \\
\hline $\begin{array}{l}\text { Deiseroth, Dieter und Heinz-Ulrich Wolf. Demokratie heute } \\
\text { 9.10, Sozialkunde, Berlin, Braunschweig: Schroedel, } 2006 .\end{array}$ & B & Unklar \\
\hline $\begin{array}{l}\text { Deiseroth, Dieter und Heinz-Ulrich Wolf. Demokratie heute, } \\
\text { Politische Bildung, Sekundarstufe I, Brandenburg, } \\
\text { Braunschweig: Schroedel, } 2009 .\end{array}$ & BB & $\begin{array}{l}\text { HS, GS, } \\
\text { RS }\end{array}$ \\
\hline $\begin{array}{l}\text { Deiseroth, Dieter und Heinz-Ulrich Wolf. Demokratie heute } \\
\text { plus 3, Politik und Wirtschaft, Hessen, Braunschweig: } \\
\text { Schroedel, } 2011 \text {. }\end{array}$ & $\mathrm{HE}$ & $\begin{array}{l}\text { HS, GS, } \\
\text { RS }\end{array}$ \\
\hline $\begin{array}{l}\text { Deiseroth, Dieter und Heinz-Ulrich Wolf. Demokratie heute, } \\
\text { Wirtschaft/Politik, Schleswig-Holstein, Braunschweig: } \\
\text { Schroedel, 2012. }\end{array}$ & $\mathrm{SH}$ & $\begin{array}{l}\text { HS, GS, } \\
\text { RS }\end{array}$ \\
\hline $\begin{array}{l}\text { Derichs, Johannes. Durchblick, Geschichte, Politik, Erdkunde } \\
\text { 9/10, Niedersachsen, Braunschweig: Westermann, } 2010 .\end{array}$ & NI & $\begin{array}{l}\text { HS, GS, } \\
\text { RS }\end{array}$ \\
\hline $\begin{array}{l}\text { Detjen, Joachim, Stefan Eggemann, Johannes Franke, Moritz- } \\
\text { Peter Haarmann, Dirk Lange, Karl-Heinz Meyer, Stephan } \\
\text { Spieker, Hermann Tallen und Friedrich-Wilhelm } \\
\text { Wedemeyer. Mensch und Politik SI, Bd. 2, Niedersachsen, } \\
\text { Braunschweig: Schroedel, 2007. }\end{array}$ & NI & GY \\
\hline
\end{tabular}




\section{(Fortsetzung)}

\begin{tabular}{|c|c|c|}
\hline Titel & $\begin{array}{l}\text { Bundes- } \\
\text { land }\end{array}$ & $\begin{array}{l}\text { Schul- } \\
\text { form }\end{array}$ \\
\hline $\begin{array}{l}\text { Detjen, Joachim, Johannes Franke, Christine Henne, Werner } \\
\text { Launhardt, Karl-Heinz Meyer, Stefan Schneider, Jörg Schulz, } \\
\text { Hermann Tallen, Friedrich-Wilhelm Wedemeyer und Jürgen } \\
\text { Westphal. Mensch und Politik, Sekundarstufe I, 9./10. } \\
\text { Schuljahr, Politik-Wirtschaft, Niedersachsen, Braunschweig: } \\
\text { Schroedel, 2012. }\end{array}$ & NI & GY \\
\hline $\begin{array}{l}\text { Eck, Guiskard, Krystyna Kusserow, Harald-Matthias } \\
\text { Neumann, Ilona Olesch und Tatjana Pfau. Terra, Für } \\
\text { Gesellschaftslehre, Geschichte und Erdkunde an Haupt- und } \\
\text { Gesamtschulen, Stuttgart: Klett, } 2009 \text {. }\end{array}$ & NI & $\begin{array}{l}\text { HS, GS, } \\
\text { RS }\end{array}$ \\
\hline $\begin{array}{l}\text { Eichner, Detlef, Olaf Eineder, Hermann Tallen und Heinz- } \\
\text { Ulrich Wolf. Demokratie heute 9/10, Politik, Realschule, } \\
\text { Niedersachsen, Braunschweig: Schroedel, } 2009 .\end{array}$ & NI & RS \\
\hline $\begin{array}{l}\text { Ernst, Christian (Hg.). Sozialkunde 9/10, Berlin, Berlin: } \\
\text { Cornelsen, } 2006 .\end{array}$ & B & Unklar \\
\hline $\begin{array}{l}\text { Ernst, Christian (Hg.). Fakt, Arbeitsbuch für Politische } \\
\text { Bildung 2, Sekundarstufe I, Brandenburg, Berlin: Cornelsen, } \\
2009 .\end{array}$ & BB & GY, GS \\
\hline $\begin{array}{l}\text { Fischer, Christine, Thomas Niedernhuber, Jakob Pritscher, } \\
\text { Martin Tuschl, Karl Uhl und Birgit Zuleger. Forum 10, } \\
\text { Sozialkunde, Realschule Bayern, Braunschweig: Westermann, } \\
\text { 2004. }\end{array}$ & BY & RS \\
\hline $\begin{array}{l}\text { Floren, Franz Josef (Hg.). Politik, 9/10, Ein Arbeitsbuch, } \\
\text { Paderborn: Schöningh, } 2003 .\end{array}$ & NI & GY \\
\hline $\begin{array}{l}\text { Floren, Franz Josef (Hg.). Politik-Wirtschaft, 9/10, } \\
\text { Arbeitsbuch, Paderborn: Schöningh, 2007. }\end{array}$ & NI & GY \\
\hline $\begin{array}{l}\text { Floren, Franz Josef (Hg.). Politik-Wirtschaft, Arbeitsbuch, für } \\
\text { Gymnasien in Nordrhein-Westfalen, Paderborn: Schöningh, } \\
2008 .\end{array}$ & NRW & GY \\
\hline $\begin{array}{l}\text { Floren, Franz Josef (Hg.). Politik-Wirtschaft, Arbeitsbuch, } \\
\text { Paderborn: Schöningh, } 2012 .\end{array}$ & NRW & GY \\
\hline $\begin{array}{l}\text { Floren, Franz Josef (Hg.). Politik-Wirtschaft, 9/10, } \\
\text { Arbeitsbuch, Paderborn: Schöningh, 2013. }\end{array}$ & NI & GY \\
\hline
\end{tabular}




\section{(Fortsetzung)}

\begin{tabular}{|l|l|l|}
\hline Titel & $\begin{array}{l}\text { Bundes- } \\
\text { land }\end{array}$ & $\begin{array}{l}\text { Schul- } \\
\text { form }\end{array}$ \\
\hline $\begin{array}{l}\text { Gaffga, Peter, Jürgen Nebel, Klaus Jörger, Alexander Oberst } \\
\text { und Alexandra Wolf (Hg.). Erdkunde, Wirtschaftskunde, } \\
\text { Gemeinschaftskunde, Realschule für Baden-Württemberg, } \\
\text { Bd. 3, Braunschweig: Westermann, 2006. }\end{array}$ & BW, SL & RS \\
\hline $\begin{array}{l}\text { Hanke, Eckhard, Erwin Henn und Friedhelm Zöllner. } \\
\text { Demokratie leben, Politik und Wirtschaft, Hessen, } \\
\text { Braunschweig: Schroedel, 2005. }\end{array}$ & HE & HS, GS \\
\hline $\begin{array}{l}\text { Heck, Stefan, Karl-Heinz Meyer, Jelko Peters und Heinz- } \\
\text { Ulrich Wolf. Politik und Wirtschaft verstehen, Nordrhein- } \\
\text { Westfalen, Braunschweig: Schroedel, 2009. }\end{array}$ & NRW & GY \\
\hline $\begin{array}{l}\text { Henninger, Sascha, Peter Kirch, Jürgen Nebel, Norma } \\
\text { Kreuzberger und Jörg Pfeiffer. Raum-Zeit-Gesellschaft 3, } \\
\text { Klassen 7-9, Rheinland-Pfalz Gesellschaftslehre, } \\
\text { Braunschweig: Westermann, 2010. }\end{array}$ & RP, SL & HS, GS, \\
\hline $\begin{array}{l}\text { Holstein, Karl-Heinz (Hg.). Fakt, 9/10, Brandenburg, } \\
\text { Politische Bildung, Berlin: Cornelsen, 2003. }\end{array}$ & RS \\
\hline $\begin{array}{l}\text { Holstein, Karl-Heinz und Joachim Amm (Hg.). Fakt, 10, } \\
\text { Gemeinschaftskunde Rechtserziehung, Sachsen, Neue } \\
\text { Ausgabe, Berlin: Cornelsen, 2006. }\end{array}$ & SN & HS, GS \\
\hline $\begin{array}{l}\text { Homann, Annette (Hg.). Anstöße 2, Politik/Wirtschaft, } \\
\text { Stuttgart/Leipzig: Klett, 2008. }\end{array}$ & NRW & GY, GS \\
\hline $\begin{array}{l}\text { Jöckel, Peter (Hg.). Mensch und Politik SI, Bd. 2, Nordrhein- } \\
\text { Westfalen, Braunschweig: Schroedel, 2004. }\end{array}$ & Versch. & GY \\
\hline $\begin{array}{l}\text { Kaminski, Hans (Hg.). Kompetenz, Politik-Wirtschaft 10, } \\
\text { Gymnasium Niedersachsen, Braunschweig: Westermann, } \\
\text { 2008. }\end{array}$ & NI & GY \\
\hline $\begin{array}{l}\text { Langhans, Ingo und Stefan Prochnow. Gemeinschaftskunde, } \\
\text { Rechtserziehung, Wirtschaft, Bd. 2, Sachsen, Donauwörth: } \\
\text { Auer, 2008. }\end{array}$ & SN & GY \\
\hline $\begin{array}{l}\text { Leicht, Jürgen und Peter Kraus (Hg.). Terra. Welt, Zeit, } \\
\text { Gesellschaft 6, Werkrealschule Baden-Württemberg, Stuttgart/ } \\
\text { Leipzig: Klett, 2008. }\end{array}$ & BW, SL & HS, GS, \\
\hline $\begin{array}{l}\text { Linder, Ronald, Harald-Matthias Neumann, Ulrich Salomon } \\
\text { und Josef Seger. Terra, Geschichte, Sozialkunde, Erdkunde 9, } \\
\text { Hauptschule Bayern, Stuttgart/Leipzig: Klett, 2008. }\end{array}$ & BY & HS, GS \\
\hline
\end{tabular}




\section{(Fortsetzung)}

\begin{tabular}{|c|c|c|}
\hline Titel & $\begin{array}{l}\text { Bundes- } \\
\text { land }\end{array}$ & $\begin{array}{l}\text { Schul- } \\
\text { form }\end{array}$ \\
\hline $\begin{array}{l}\text { Lübbert, Heinrich, Harald-Matthias Neumann und Thomas } \\
\text { Specht. Anstöße 3, Politik, Stuttgart/Leipzig: Klett, } 2012 .\end{array}$ & NRW & Unklar \\
\hline $\begin{array}{l}\text { Lüer, Rolf-Dieter und Eckhardt Wansleben (Hg.). Anstöße } \\
\text { Politik, Politik und Wirtschaft, Niedersachsen, Stuttgart } \\
\text { [u. a.]: Klett, 2006. }\end{array}$ & NI & RS \\
\hline $\begin{array}{l}\text { Mack, Andreas und Jürgen Fehn. Demokratie verpflichtet, } \\
\text { Lehr- und Arbeitsbuch für den Sozialkundeunterricht an } \\
\text { sechsstufigen Realschulen in Bayern, München: Oldenbourg, } \\
2003 \text {. }\end{array}$ & BY & RS \\
\hline $\begin{array}{l}\text { Mack, Andreas und Jürgen Fehn. Demokratie verpflichtet, } \\
\text { Lehr- und Arbeitsbuch für den Sozialkundeunterricht an } \\
\text { Realschulen in Bayern, München: Oldenbourg, } 2011 .\end{array}$ & $\mathrm{BY}$ & RS \\
\hline $\begin{array}{l}\text { Mattes, Wolfgang (Hg.). Team 2, Arbeitsbuch für den } \\
\text { Politikunterricht, Paderborn: Schöningh, } 2004 .\end{array}$ & $\begin{array}{l}\text { Versch. } \\
\text { BL }\end{array}$ & GY, GS \\
\hline $\begin{array}{l}\text { Mattes, Wolfgang (Hg.). Team 9, Arbeitsbuch Politik und } \\
\text { Wirtschaft, Gymnasium Nordrhein-Westfalen, Paderborn: } \\
\text { Schöningh, } 2010 .\end{array}$ & NRW & GY \\
\hline $\begin{array}{l}\text { Mattes, Wolfgang (Hg.). Team 9/10, Arbeitsbuch für Politik, } \\
\text { Realschule Niedersachsen, Paderborn: Schöningh, } 2010 .\end{array}$ & NI & RS \\
\hline $\begin{array}{l}\text { Mattes, Wolfgang (Hg.). Team 2, Arbeitsbuch GWG } \\
\text { Gemeinschaftskunde/Wirtschaft, Gymnasium Baden- } \\
\text { Württemberg, Paderborn: Schöningh, } 2012 .\end{array}$ & $\mathrm{BW}, \mathrm{SL}$ & GY \\
\hline $\begin{array}{l}\text { Mattes, Wolfgang (Hg.). Team 3, Arbeitsbuch für Politik und } \\
\text { Wirtschaft, Paderborn: Schöningh, } 2012 .\end{array}$ & NRW & RS \\
\hline $\begin{array}{l}\text { Mattheus, Stephan, Harald-Matthias Neumann und Thomas } \\
\text { Specht. Anstöße 2, Politik-Wirtschaft, Stuttgart/Leipzig: } \\
\text { Klett, } 2013 .\end{array}$ & NI & GY, GS \\
\hline $\begin{array}{l}\text { Nebel, Jürgen, Georg Baumbach, Knut Engeler, Winfried } \\
\text { Groß, Nittert Janssen, Norma Kreuzberger, Heike Schaadt, } \\
\text { Jens Siebert und Alexander Wiebel. Gesellschaft bewusst 3, } \\
\text { Ausgabe Nord, Braunschweig: Westermann, 2011. }\end{array}$ & NI & GY \\
\hline $\begin{array}{l}\text { Nebel, Jürgen, Peter Gaffga, Peter Kirch, Norma Kreuzberger, } \\
\text { Friedrich Pauly, Roland Theophil und Roland Widmann. } \\
\text { Welt-Zeit-Gesellschaft 4, Baden-Württemberg, Hauptschule, } \\
\text { Braunschweig: Westermann, 2006. }\end{array}$ & BW, SL & $\begin{array}{l}\text { HS, GS, } \\
\text { RS }\end{array}$ \\
\hline
\end{tabular}




\section{(Fortsetzung)}

\begin{tabular}{|c|c|c|}
\hline Titel & $\begin{array}{l}\text { Bundes- } \\
\text { land }\end{array}$ & $\begin{array}{l}\text { Schul- } \\
\text { form }\end{array}$ \\
\hline $\begin{array}{l}\text { Neumann, Harald-Matthias (Hg.). Terra, 9/10, Für } \\
\text { Gesellschaftslehre, Geschichte und Erdkunde an Haupt- und } \\
\text { Gesamtschulen, Stuttgart: Klett, } 2007 .\end{array}$ & NI & HS, GS \\
\hline $\begin{array}{l}\text { Neumann, Harald-Matthias und Thomas Specht. Anstöße 2, } \\
\text { Politik, Stuttgart: Klett, } 2010 .\end{array}$ & NI & GY, GS \\
\hline $\begin{array}{l}\text { Riedel, Hartwig (Hg.). Politik \& Co. 2, Sozialkunde und } \\
\text { Wirtschaft für das Gymnasium, Bamberg: C.C. Buchner, } 2005 .\end{array}$ & $\begin{array}{l}\text { Versch. } \\
\text { BL }\end{array}$ & GY \\
\hline $\begin{array}{l}\text { Riedel, Hartwig (Hg.). Politik \& Co., Politik-Wirtschaft für } \\
\text { das Gymnasium, Niedersachsen, Bamberg: C.C. Buchner, } \\
\text { 2007. }\end{array}$ & $\mathrm{NI}$ & GY \\
\hline $\begin{array}{l}\text { Riedel, Hartwig (Hg.). Politik \& Co., Sozialkunde für das } \\
\text { Gymnasium, Rheinland-Pfalz, Bamberg: C.C. Buchner, } 2011 .\end{array}$ & $\mathrm{RP}, \mathrm{SL}$ & GY \\
\hline $\begin{array}{l}\text { Riedel, Hartwig (Hg.). Politik \& Co. 2, Politik/Wirtschaft für } \\
\text { das Gymnasium, Nordrhein-Westfalen, Bamberg: C.C. } \\
\text { Buchner, 2011. }\end{array}$ & $\begin{array}{l}\text { Versch. } \\
\text { BL }\end{array}$ & GY \\
\hline $\begin{array}{l}\text { Riedel, Hartwig (Hg.). Politik \& Co. 2, Politik und Wirtschaft } \\
\text { für das Gymnasium, Hessen, Bamberg: C.C. Buchner, } 2012 .\end{array}$ & $\mathrm{HE}$ & GY \\
\hline $\begin{array}{l}\text { Riedel, Hartwig (Hg.). Politik \& Co. 2, Politik-Wirtschaft für } \\
\text { das Gymnasium, Niedersachsen, Bamberg: C.C. Buchner, } \\
\text { 2013. }\end{array}$ & NI & GY \\
\hline $\begin{array}{l}\text { Rudyk, Ellen (Hg.). Politik und Wirtschaft 9/10, Gymnasium } \\
\text { Niedersachsen, Berlin: Cornelsen, 2007. }\end{array}$ & NI & GY \\
\hline $\begin{array}{l}\text { Rudyk, Ellen (Hg.). Politik und Wirtschaft 9/10, Gymnasium } \\
\text { Schleswig-Holstein, Berlin: Cornelsen, } 2009 .\end{array}$ & $\mathrm{SH}$ & GY \\
\hline $\begin{array}{l}\text { Sauer, Nicole, Hartmut Sperling, Heinz-Ulrich Wolf und } \\
\text { Reinhard Ziegler. Demokratie heute 10, Politik und Wirtschaft, } \\
\text { Realschule Hessen, Braunschweig: Schroedel, } 2005 .\end{array}$ & $\mathrm{HE}$ & $\mathrm{RS}$ \\
\hline $\begin{array}{l}\text { Schreder, Gabriele und Karl-Heinz Holstein (Hg.). Politik und } \\
\text { Wirtschaft, 9/10, Berlin: Cornelsen, } 2005 .\end{array}$ & $\mathrm{HE}$ & GY \\
\hline $\begin{array}{l}\text { Sperling, Hartmut, Heinz-Ulrich Wolf und Julius Wöppel. } \\
\text { Demokratie heute 10, Gemeinschaftskunde/Rechtserziehung, } \\
\text { Mittelschule Sachsen, Hannover: Schroedel, } 2003 .\end{array}$ & SN & HS, GS \\
\hline
\end{tabular}


(Fortsetzung)

\begin{tabular}{|l|l|l|}
\hline Titel & $\begin{array}{l}\text { Bundes- } \\
\text { land }\end{array}$ & $\begin{array}{l}\text { Schul- } \\
\text { form }\end{array}$ \\
\hline $\begin{array}{l}\text { Tieke, Ralf, Matthias Bahr, Uwe Hofemeister, Klaus Langer, } \\
\text { Martin Lücke, Ulrike Lüthgen-Frieß, Klaus Maiwald, } \\
\text { Wolfgang Pankratz und Carmen Weiß. Durchblick, } \\
\text { Geschichte und Politik 9/10, Niedersachsen, Braunschweig: } \\
\text { Westermann, 2010. }\end{array}$ & $\begin{array}{l}\text { HS, GS, } \\
\text { RS }\end{array}$ \\
\hline $\begin{array}{l}\text { Tieke, Ralf, Matthias Bahr, Monika Lenniger, Martin Lücke, } \\
\text { Klaus Maiwald, Wolfgang Pankratz, Hildegard Wacker, } \\
\text { Carmen Weiß und Gerald Wieziolkowski. Durchblick, } \\
\text { Geschichte und Politik 9/10, Realschule Niedersachsen, } \\
\text { Braunschweig: Westermann, 2005. }\end{array}$ & NI & HS, GS, \\
\hline $\begin{array}{l}\text { Wolf, Heinz-Ulrich (Hg.). Demokratie heute 9/10, } \\
\text { Sozialkunde, Mecklenburg-Vorpommern, Braunschweig: } \\
\text { Schroedel, 2007. }\end{array}$ & MV & $\begin{array}{l}\text { HS, GS, } \\
\text { RS }\end{array}$ \\
\hline $\begin{array}{l}\text { Wuttke, Carola (Hg.). Politik-Wirtschaft, Lehrbuch für die } \\
\text { Klasse 9/10, Gymnasium Niedersachsen, Berlin: Duden- } \\
\text { Paetec Schulbuchverlage, 2007. }\end{array}$ & NI & GY \\
\hline
\end{tabular}


Open-Access-Publikation im Sinne der CC-Lizenz BY 4.0

(C) 2021, Vandenhoeck \& Ruprecht $\mathrm{GmbH} \&$ Co. KG, Göttingen ISBN Print: 9783847112655 - ISBN E-Lib: 9783737012652 